\title{
WestVirginiaUniversity
}

THE RESEARCH REPOSITORY @ WVU

Graduate Theses, Dissertations, and Problem Reports

2014

\section{Plantwide Control System Design for IGCC Power Plants with CO2 Capture}

Dustin Douglas Jones

West Virginia University

Follow this and additional works at: https://researchrepository.wvu.edu/etd

\section{Recommended Citation}

Jones, Dustin Douglas, "Plantwide Control System Design for IGCC Power Plants with CO2 Capture" (2014). Graduate Theses, Dissertations, and Problem Reports. 530.

https://researchrepository.wvu.edu/etd/530

This Dissertation is protected by copyright and/or related rights. It has been brought to you by the The Research Repository @ WVU with permission from the rights-holder(s). You are free to use this Dissertation in any way that is permitted by the copyright and related rights legislation that applies to your use. For other uses you must obtain permission from the rights-holder(s) directly, unless additional rights are indicated by a Creative Commons license in the record and/ or on the work itself. This Dissertation has been accepted for inclusion in WVU Graduate Theses, Dissertations, and Problem Reports collection by an authorized administrator of The Research Repository @ WVU.

For more information, please contact researchrepository@mail.wvu.edu. 
Plantwide Control System Design for IGCC Power Plants with CO2 Capture

Dustin Douglas Jones

Thesis submitted to the College of Engineering and Mineral Resources at West Virginia University in partial fulfillment of the requirements for the degree of

Doctor of Philosophy

In

Chemical Engineering

Dr. Debangsu Bhattacharyya

Dr. Muhammad A. Choudhry

Dr. Richard Turton

Dr. Stephen E. Zitney

Dr. John Zondlo

Department of Chemical Engineering

Morgantown, West Virginia

2014

Keywords: IGCC, Optimization, Control, Primary Controlled Variables, Secondary Controlled Variables 


\title{
ABSTRACT \\ Steady State and Dynamic Modeling of the Modified Claus Process as part of an IGCC Power Plant
}

\author{
Dustin Douglas Jones
}

In this paper, a systematic approach to design the control system of a commercial-scale integrated gasification combined cycle (IGCC) power plant with $\mathrm{CO}_{2}$ capture is considered. The control system design is developed with the objective of optimizing a desired scalar function while satisfying operational and environmental constraints in the presence of measured and unmeasured disturbances. Various objective functions can be considered for the control system design such as maximization of profit, maximization of the power produced, or minimization of the auxiliary power consumed in the plant. The design of such a control system can make the IGCC plant suitable to play an active role in the smart grid era by enabling operation in the loadfollowing mode as demand for electricity from the grid fluctuates over time. In addition, other penalty functions such as emission penalties for $\mathrm{CO}_{2}$ or other criteria pollutants can be considered in the control system design.

The control system design is performed in two stages. In the first stage, a top-down analysis is used to generate a list of controlled, manipulated, and disturbance variables considering a scalar operational objective and other process constraints. In this section, innovative methods devised for primary and secondary controlled variable selection will be discussed.Exploiting these results, the second stage uses a bottom-up approach for simultaneous design of the control structure and the controllers. In this section, a novel means of control structure design has been proposed.

In this research, the proposed two-stage control system design approach is applied to the IGCC's acid gas removal (AGR) process which uses the physical solvent Selexol ${ }^{\mathrm{TM}}$ to selectively remove $\mathrm{CO}_{2}$ and $\mathrm{H}_{2} \mathrm{~S}$ from the shifted syngas. Aspen Plus Dynamics ${ }^{\circledR}$ is used to develop the AGR process model while MATLAB ${ }^{\circledR}$ is used to perform the control system design. This work has shown the proposed design procedure for plantwide control yields an optimal control structure. Additionally, the methods proposed in this work for primary and secondary controlled variable selection yield controlled variables which balance economic and control performance. Finally, the method proposed for control structure design has been found to yield a control structure that balance the control performance with controller complexity. 


\section{Table of Content}

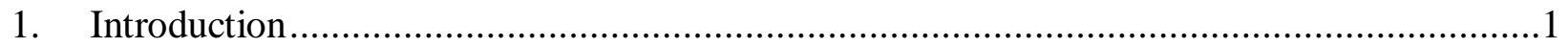

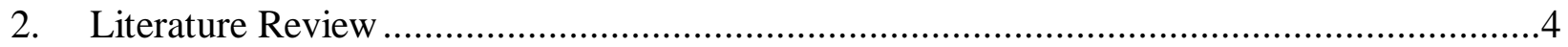

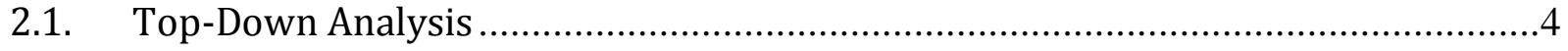

2.1.1. Definition of Operational Objective and Constraints..........................................

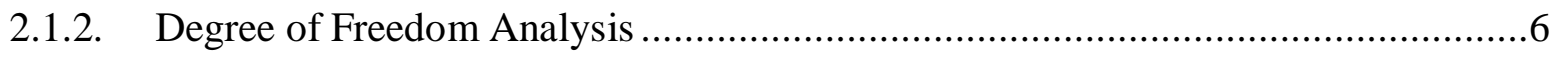

2.1.3. Primary Controlled Variable Selection ................................................................

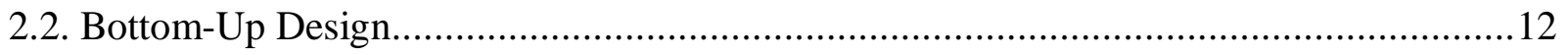

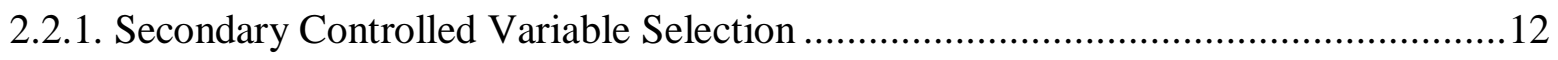

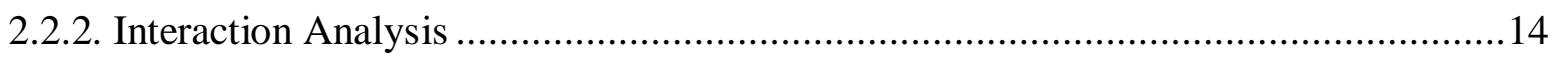

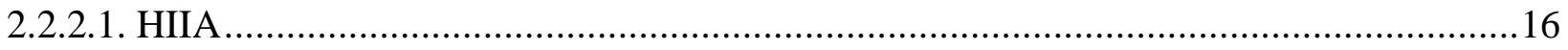

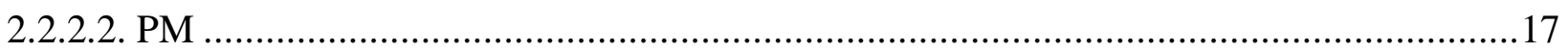

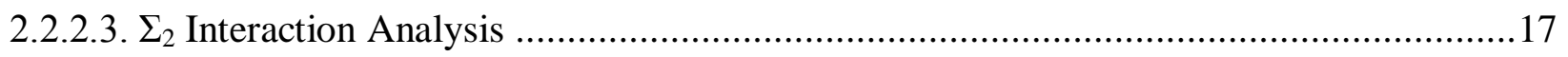

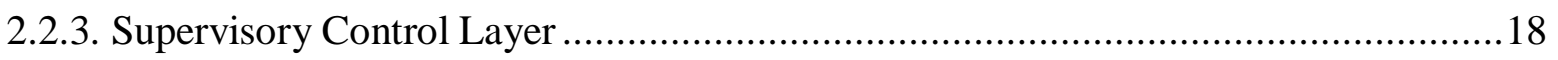

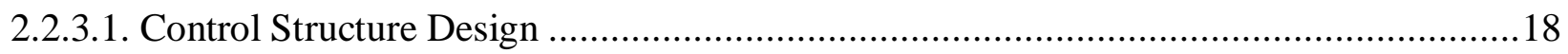

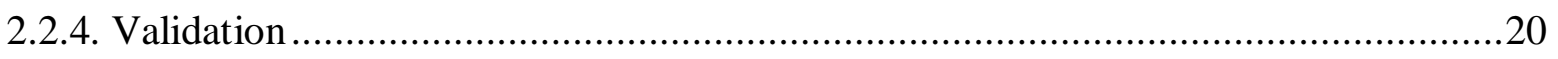

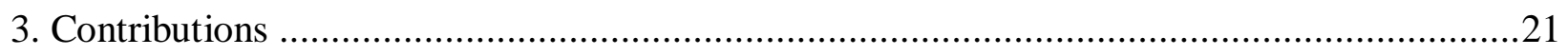

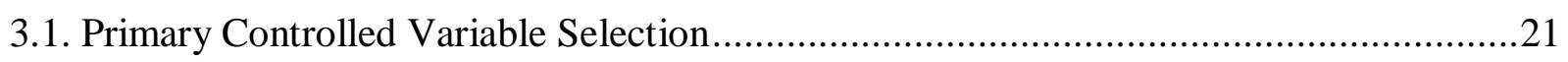

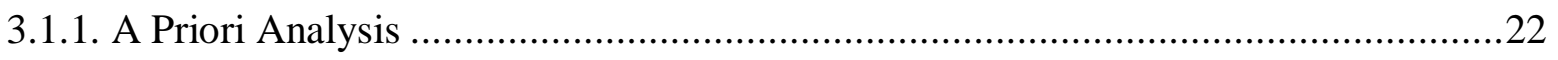

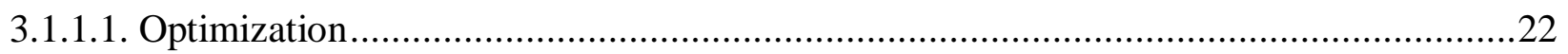

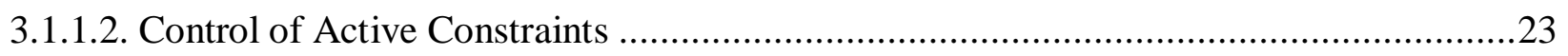

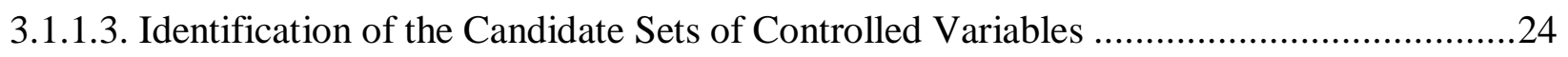

3.1.2. Selection of the Pareto-Optimal Controlled Variable Sets ........................................25

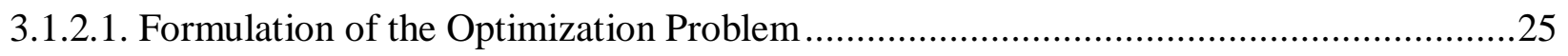

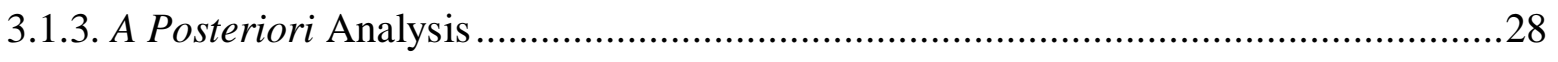

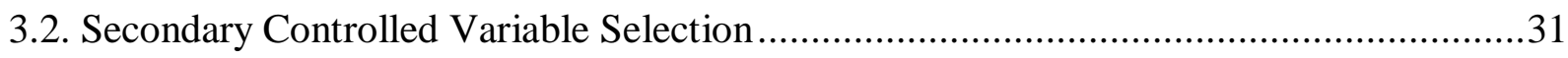

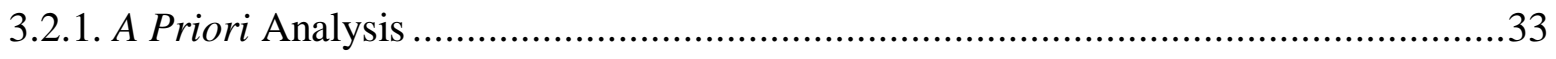

3.2.1.1. Identification of a Candidate Set of Secondary Controlled Variables and Disturbances .33

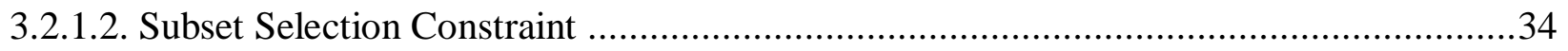

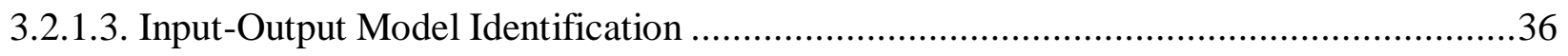

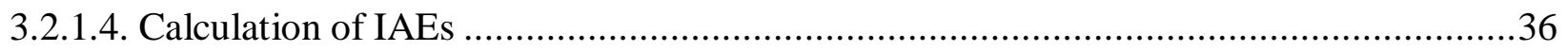

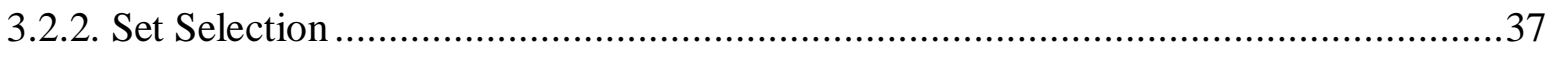

3.2.2.1. Servo and Regulatory Control of Regulatory Control Layer ..........................................38

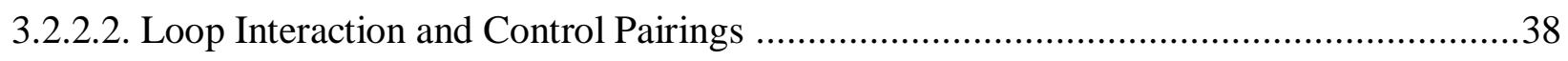

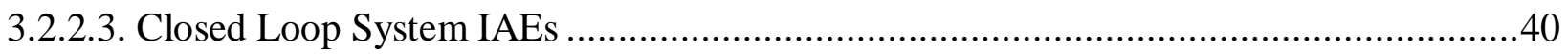


3.2.2.4. Formulation of the Optimization Problem ...............................................................41

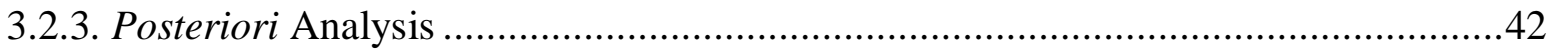

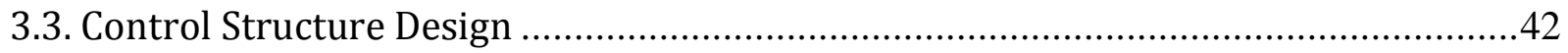

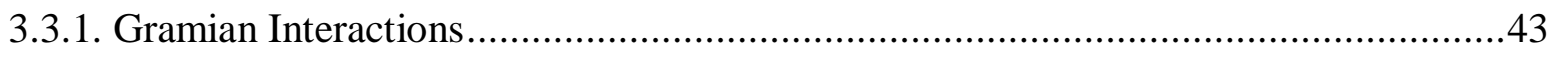

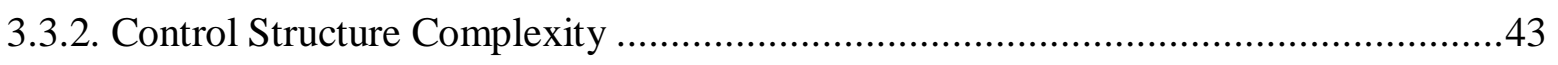

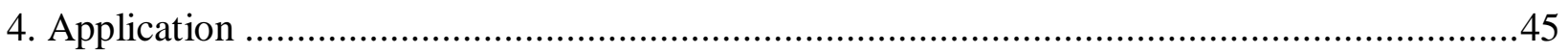

4.1. Acid Gas Removal (AGR) Unit ........................................................................46

4.1.1. Top-Down Analysis ...................................................................................... 47

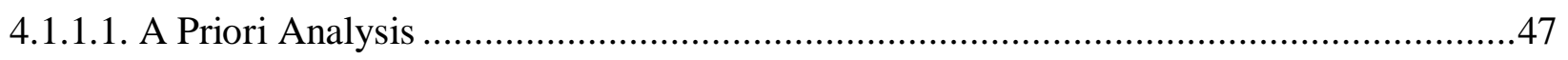

4.1.1.1.1. Optimization ....................................................................... 47

4.1.1.1.2. Control of Active Constraints ................................................................51

4.1.1.1.3. Identification of the Candidate Sets of Controlled Variables...................51

4.1.1.2. Selection of the Pareto-Optimal Controlled Variable Sets ........................................54

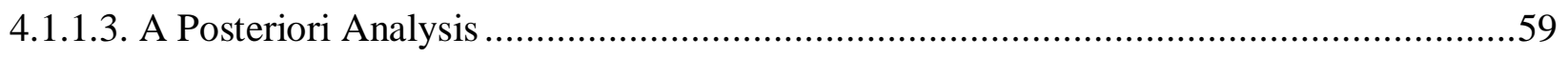

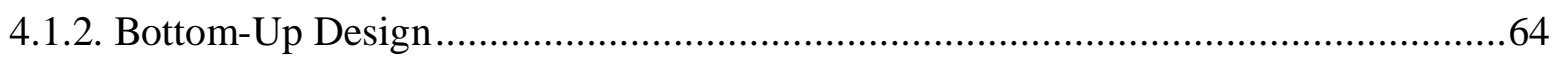

4.1.2.1. Secondary Controlled Variable Selection .........................................................64

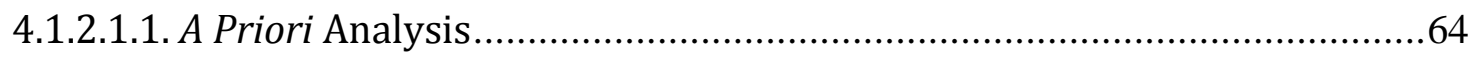

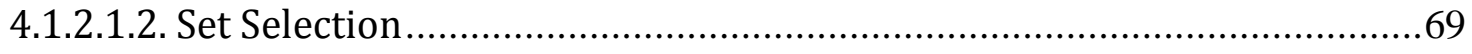

4.1.2.1.3. Posteriori Analysis ............................................................ 73

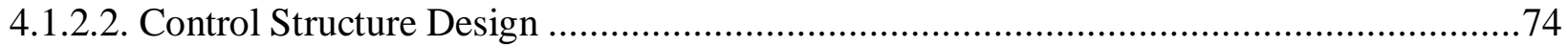

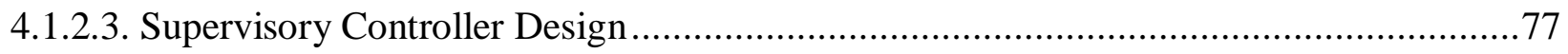

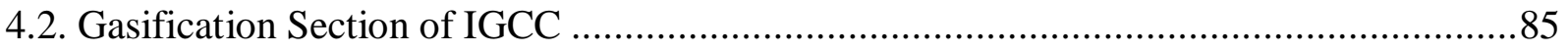

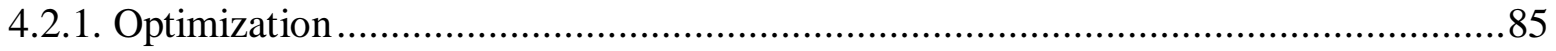

4.2.2. Primary Controlled Variable Selection ....................................................... 86

4.2.3. Secondary Controlled Variable Selection .................................................... 87

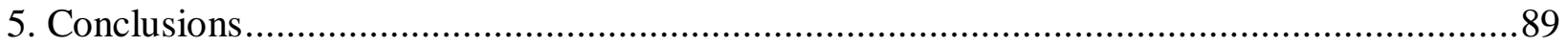

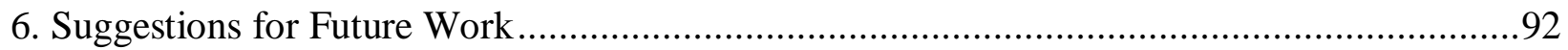

6.1 Primary Controlled Variable Selection.................................................................92

6.2 Secondary Controlled Variables...................................................................... 92

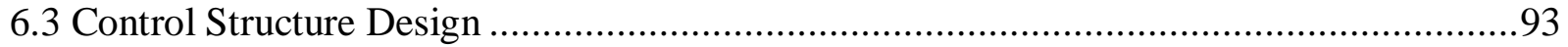

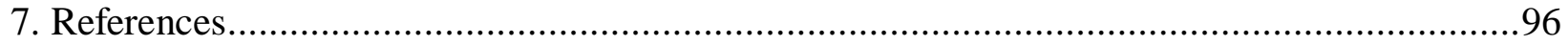

Appendix A: Parallelized Bi-Directional Branch and Bound Algorithm ..............................98 


\section{List of Tables}

Table 1. Plantwide control structure design procedure, top-down anlaysis . . . . . . . . . .5

Table 2. Plantwide control structure design procedure, bottom-up design . . . . . . . . . . .6

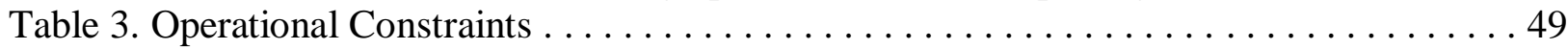

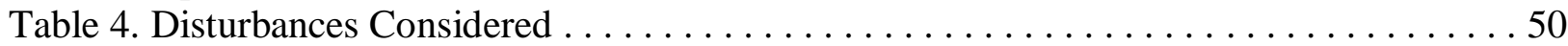

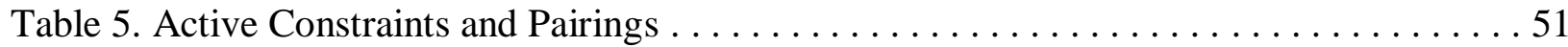

Table 6. List of Candidate Controlled Variables . . . . . . . . . . . . . . . . . . . . 53

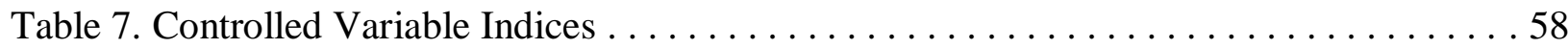

Table 8. Controlled Variable Sets Arranged in Descending Cost Performance . . . . . . . . . . .59

Table 9. Economic Loss $\left[\$ h^{-1}\right]$ of Controlled Variable Sets $\ldots \ldots \ldots \ldots \ldots \ldots \ldots$

Table 10. Effect of Disturbances on Controllability of Controlled Variable Sets [minimum

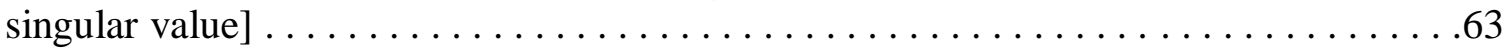

Table 11. Controllability [minimum singular value] of C9 and C10 with Constant Setpoint

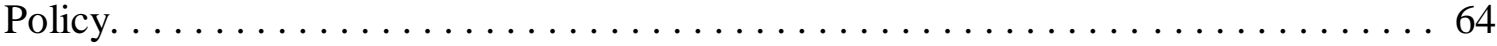

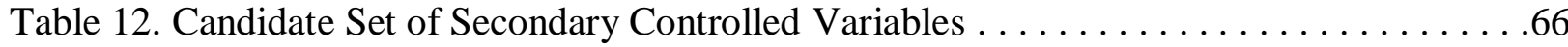

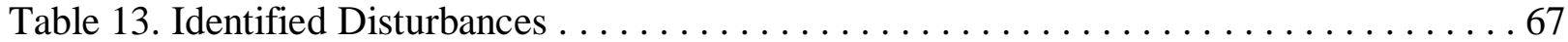

Table 14. Manipulated Variables for Control of Secondary Controlled Variables . . . . . . . . 68

Table 15. Disturbance Variables Considered during Secondary Controlled Variable Selection 68

Table 16. Controller Gain Table . . . . . . . . . . . . . . . . . . . . . . . . . . . . . . . . 69

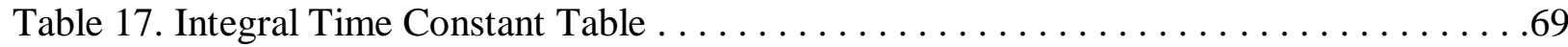

Table 18. Results of optimization with no constraint on servo performance $\ldots \ldots \ldots \ldots \ldots 71$

Table 19. Results of optimization with constraint on servo performance $\ldots \ldots \ldots \ldots \ldots 72$

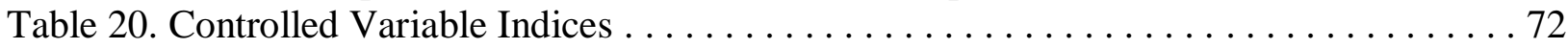

Table 21. Ultimate Pairings of Manipulated Variables to Primary Controlled for each Category

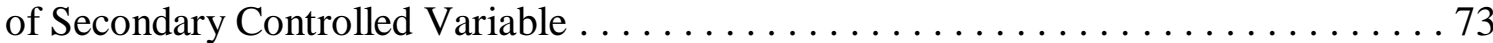

Table 22. Off-design control performance regulatory control layer . . . . . . . . . . . . . 74

Table 23. Numerical Derivative of Control Performance with respect to Controller Complexity

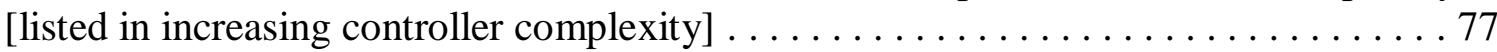

Table 24. Comparison of Initial LMPC to PID for Three Disturbances . . . . . . . . . . . .82

Table 25. Comparison of ISE Values for a -20\% Step Change in Syngas Flow . . . . . . . . . 85

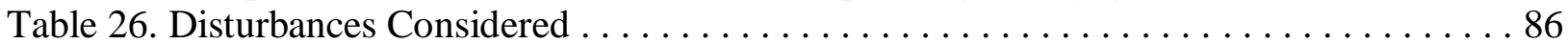

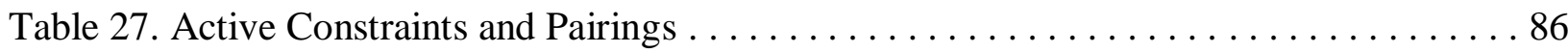

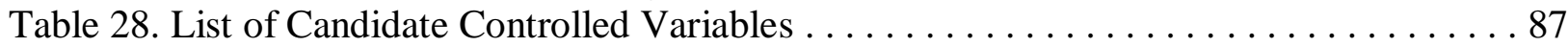

Table 29. List of Candidate Secondary Controlled Variable . . . . . . . . . . . . . . 88 


\section{List of Figures}

Figure 1. Typical Control Hierarchy in a Chemical Plant . . . . . . . . . . . . . . .2

Figure 2. Example of Degree of Freedom Analysis $\ldots \ldots \ldots \ldots \ldots \ldots \ldots \ldots \ldots \ldots$

Figure 3. Comparison of constant setpoint policy with two controlled variables sets where $\mathrm{d}^{*}$ is

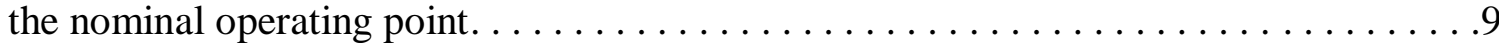

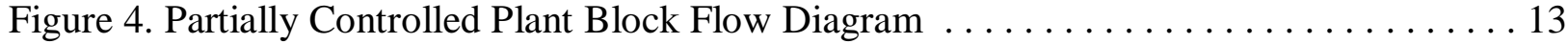

Figure 5. Block flow diagram of cascaded primary control variable loop . . . . . . . . . . 37

Figure 6. Block Flow Diagram of IGCC with Carbon Capture . . . . . . . . . . . . . . 46

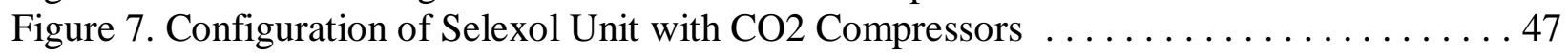

Figure 8. Pareto Solutions for Controlled Variable Selection Problem . . . . . . . . . . . . . 57

Figure 9. Effect of Syngas Flowrate on Economic Loss of Selected Controlled Variables. . . . 61

Figure 10. Effect of CO2 Capture Requirement on Economic Loss of Selected Controlled

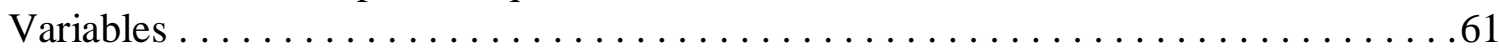

Figure 11. Effect of Syngas Throughput on the Gain of Controlled Variable . . . . . . . . 63

Figure 12. Pareto Set for Control Structure using HIIA Interaction Measure . . . . . . . 75

Figure 13. Pareto Set for Control Structure using PM Interaction Measure . . . . . . . . . 76

Figure 14. Pareto Set for Control Structure using $\Sigma 2$ Interaction Measure . . . . . . . . . . 76

Figure 15. PRBS Input Used for Semilean Solvent Flow . . . . . . . . . . . . . . 78

Figure 16. Effect of PRBS input signal on CO2 Capture and Comparison with Identified Model .

Figure 17. Effect of PRBS input signal on $\mathrm{CO} 2$ Vapor Composition in $\mathrm{CO} 2$ Absorber and

Comparison with Identified Model . . . . . . . . . . . . . . . . . . . 79

Figure 18. Effect of PRBS input signal on Solvent Composition in H2S Absorber and

Comparison with Identified Model . . . . . . . . . . . . . . . . . . . . . . 79

Figure 19. Effect of PRBS input signal on H2S Capture and Comparison with Identified Model .

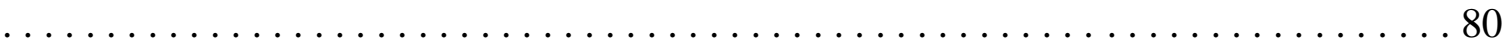

Figure 20. Effect of PRBS input signal on Water Composition of Solvent and Comparison with

Identified Model . . . . . . . . . . . . . . . . . . . . . 80

Figure 21. Effect of PRBS input signal on H2S Purity to Claus Unit and Comparison with

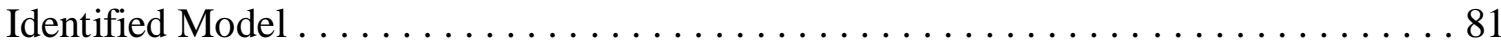

Figure 22. Economic Loss as a Function of Time due to $-20 \%$ Step in Syngas Flow . . . . . . 83

Figure 23. CO2 Capture Fraction after $-20 \%$ Step Change in Syngas Flow . . . . . . . . . . 83

Figure 24. H2S Purity to Claus after $-20 \%$ Step Change in Syngas Flow. . . . . . . . . . . . . 84

Figure 25. CO2 Vapor Fraction in CO2 Absorber after -20\% Step Change in Syngas Flow . . 84

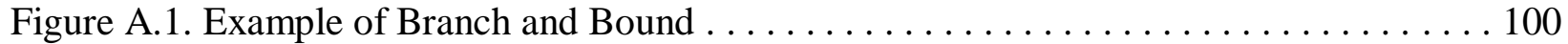

Figure A.2. Example of Pruning Rule for finding the Pareto Front . . . . . . . . . . . 103

Figure A.3. Parallelizing Method for Branch and Bound Algorithm . . . . . . . . . . . . . 104

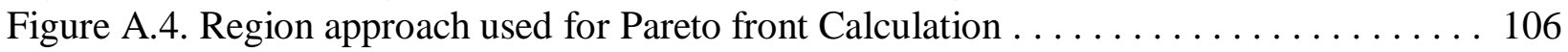




\section{Introduction}

Designing an optimal control system for an IGCC plant with $\mathrm{CO}_{2}$ capture addresses the challenge of efficiently operating and controlling coal-fed IGCC plants with the desired extent of $\mathrm{CO}_{2}$ capture in the face of disturbances without violating operational and environmental constraints. The control system design needs to optimize a desired scalar function while satisfying all the operational and environmental constraints in the presence of measured and unmeasured disturbances. Various objective functions can be considered for the control system design such as maximization of profit, maximization of the power produced, or minimization of the auxiliary power. The design of such a control system makes the plant suitable to play an active role in the smart grid era. In addition, other penalty function(s) such as emission penalties for $\mathrm{CO}_{2}$ or other criteria pollutants may be considered in the framework. This approach can be followed for control system design of future IGCC plants at the grassroots level as well as for retrofitting existing plants with suitable modifications. More specifically, the approach will be applicable for any number of large scale plants.

The control system design will be performed in two stages. In the first stage, a top-down approach will be taken to generate a list of manipulated, control, and disturbance variables considering a scalar operation objective and other process constraints. In the second stage, a bottom-up approach will be used for simultaneous design of the control structure and the controllers taking into account the results from the previous stage. The regulatory control layer will be designed for both servo control and disturbance rejections. Traditional, as well as advanced, PID controllers will be designed in this layer. In the supervisory control layer, both centralized and decentralized controls will be explored. For centralized control, linear model predictive control (MPC) will be evaluated where the process models will be identified from the first principles dynamic model (of the plant). Finally, an optimization layer will be designed that can satisfy the operational objective by utilizing the primary controlled variables as degrees of freedom. The design procedure will first be developed and applied to the Selexol unit of an IGCC plant.

In practice, the control system of a chemical plant is divided into multiple control layers which are separated by their respective time scales. Figure 1 shows five of these control layers and how they are connected (Skogestad, 2004). This work will be concerned with the design of the lower 
three levels. The regulatory control level is required primarily for the stabilization of the plant and operates in the time scale of seconds. The supervisory control layer is required to maintain desired process outputs and operates in the time scale of minutes. The local optimizer is used for determining the optimal process outputs and operates in the time scale of hours. These control layers are connected by the set points of the lower level controllers. The local optimizer sets the set points of the supervisory control layer that determines the set points of the regulatory control layer to attain the desired output.

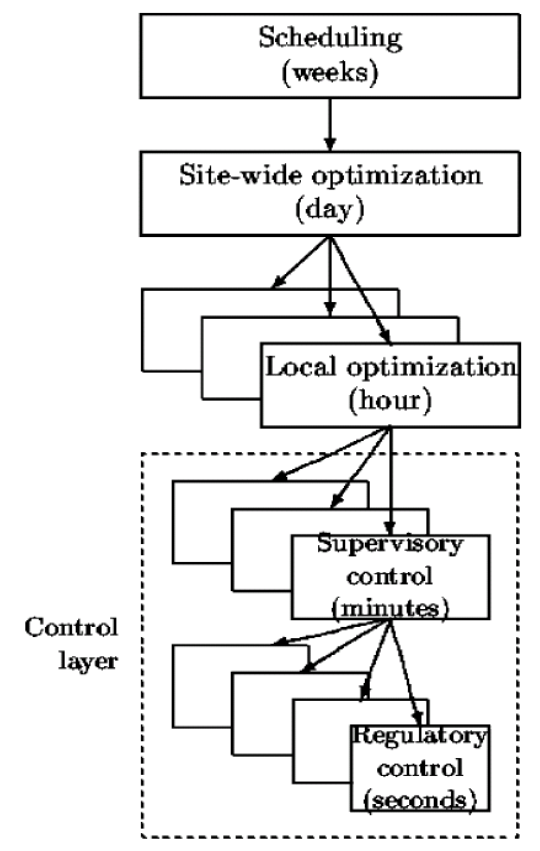

Figure 1. Typical Control Hierarchy in a Chemical Plant (Skogestad, 2004)

Plantwide control is described by Skogestad (Skogestad, 2004) as dealing with the structural decisions required before controllers are designed. Skogestad breaks this process into several tasks:

1. $\quad$ selection of manipulated variables $m$ (inputs)

2. selection of controlled variables (outputs)

3. selection of additional measurements for control purposes, including stabilization

4. selection of control configuration (the structure of the overall controller that interconnects the controlled, manipulated, and measured variables)

5. selection of controller type (PID, decoupler, linear-quadratic-Gaussian control, etc.) 
Methods exist for some of these tasks, however, generally these tasks are undertaken following a heuristic method. Foss (Foss, 1973) describes the problem in the following way:

"The central issue to be resolved by the new theories is the determination of the control system structure. Which variables should be measured which inputs should be manipulated and which links should be made between the two sets? There is more than a suspicion that the work of a genious is needed here, for without it the control configuration problem will likely remain in a primitive, hazily stated and wholly unmanageable form. The gap is present indeed, but contrary to the views of many, it is the theoretician who must close it."

Though this area of research has been active for the last decade, many gaps still exists which limit the generic applicablity. The objective of this research is to identify these gaps and to devise new procedures which will enhance the design procedure and allow it to become more generically applicable. These include the identification of candidate controlled variables, the selection criteria of primary controlled variables, and how one is to connect manipulated variables with controlled variables, just to name a few. Additionally, it is desired to determine how these methods can be applied to large-scale, highly nonlinear systems where the linear models used for the design may not be applicable. 


\section{Literature Review}

A design procedure proposed by Skogestad (Skogestad, 2004) is outlined in Tables 1 and 2. The design procedure that will be developed in this work is based largely on the design procedure proposed by Skogestad. This procedure is broken into two stages, a top-down analysis and a bottom-up design. The top-down analysis deals with the definition of an operational objective and process constraints. Additionally, it is at this stage that input and output variables are selected. During the bottom-up design stage, the control layers of the control system are designed sequentually.

\subsection{Top-Down Analysis}

During the top-down analysis portion of the design, no controller design is undertaken. This analysis is concerned with identification of operational regions of the process and determining what should be controlled within the process.

\subsubsection{Definition of Operational Objective and Constraints}

The first step in the top-down analysis is the definition of an operational objective and operational constraints. This operational objective must be a scalar cost function that must be minimized. The operational objective could be minimization of the cost of operation, auxiliary power usage, total power output, or any other objective. The constraints of the process can be related to operational constraints, e.g., maximum allowable pressure of a vessel, or environmental constraints, e.g., maximum $\mathrm{CO}_{2}$ emissions. 
Table 1. Plantwide control structure design procedure, top-down anlaysis (Skogestad , 2004) Step Comment Proposed Modifications

(I)Top - down analysis

1. Definition of operational

objectives

Identify operational constraints, and

a scalar cost function $\mathrm{J}$ to be

minimized

\begin{tabular}{|c|c|c|}
\hline $\begin{array}{l}\text { 2. Manipulated variables and degrees of freedom } \\
\text { Identify dynamic and steady-state degrees of freedom }\end{array}$ & $\begin{array}{l}\text { May need extra equipment of } \\
\text { there are too few DOF's }\end{array}$ & \\
\hline $\begin{array}{l}\text { 3. Primary controlled variables } \\
\text { Which variables should we control? } \\
\text { - Active Constraints } \\
\text { - With remaining control variables, control } \\
\text { variables for which constant set points give } \\
\text { small economic loss when disturbances occur }\end{array}$ & $\begin{array}{l}\text { Steady-state economic } \\
\text { analysis: } \\
\text { - } \\
\text { - } \\
\text { cofine constraint and } \\
\text { Optimization w.r.t } \\
\text { steady-state DOF's } \\
\text { for various } \\
\text { disturbances } \\
\text { (identifies active } \\
\text { constraints) } \\
\text { Evaluation of loss } \\
\text { with constant set } \\
\text { points } \\
\end{array}$ & $\begin{array}{l}\text { Multi-objective optimization } \\
\text { consisting of an economic } \\
\text { performance function and a } \\
\text { controllability function. If not } \\
\text { computationally prohibitive, } \\
\text { consider both controlled variables } \\
\text { and manipulated variables selection } \\
\text { (simultaneous design of regulatory } \\
\text { control layer) }\end{array}$ \\
\hline $\begin{array}{l}\text { 4. Production rate } \\
\text { Where should the production rate be set? (Very } \\
\text { important choice as it determines the structure of } \\
\text { remaining inventory control system) }\end{array}$ & $\begin{array}{l}\text { Optimal location follows } \\
\text { from steady-state } \\
\text { optimization (step 3), but } \\
\text { may move depending on } \\
\text { operation }\end{array}$ & $\begin{array}{l}\text { This should be undertaken during } \\
\text { step } 3 \text { unless a bottleneck is present }\end{array}$ \\
\hline
\end{tabular}


Table 2. Plant-wide control structure design procedure, bottom-up design (Skogestad, 2004)

Comment Proposed Modifications

\begin{tabular}{l} 
Step \\
(II) Bottom-up design \\
\hline 5. Regulatory control layer \\
5.1 Stabilization \\
5.2 Local disturbance rejection \\
Purpose: "Stabilize" the plant using low-complexity \\
controllers (single-loop PID controllers) such that 1) the \\
plant does not drift too far away from its nominal operating \\
point and 2) the supervisory layer (or the operators) can \\
handle the effect of disturbances on the primary outputs \\
Main Structural issue: What more should we control \\
- Select secondary controlled variables \\
(measurements) \\
$\quad$ Pairing these with manipulated variables avoiding \\
$\quad$ manipulated variables that saturate
\end{tabular}

5.1 Pole vector analysis for selecting measured variables and manipulated inputs for stabilizing control

5.2 Partially controlled plant analysis. Control secondary measurements so that the sensitivity of states to disturbances is small at intermediate frequencies Model Requirement: Linear multivariable dynamic model

6. Supervisory control layer

Purpose: Keep primary controlled variables at optimal set points using degrees of freedom (inputs) the set points for the regulatory layer and any unused manipulated variables. Main Structural issue: Decentralized or multivariable control?

6a. Decentralized (single-loop) control

- May use simple PI or PID controllers

- Structural issues: choose input-output pairing

Possibly with addition of feed-forward and ratio control

Pairing analysis: Pair on RGA close to identity matrix at crossover frequency, provided not negative at steady-state.

Use CLDG for more detailed analysis

6b. Multivariable control

1. Use for interacting processes and for easy handling of feed-forward control

2. Use MPC with constraints handling for moving smoothly between changing active constraints (avoids logic needed in decentralized scheme)

7. Optimization layer

Model Requirements:

Nonlinear steady-state model, plus costs and set points for controlled variables

Main structural issue: Do we need real-time optimization (RTO)?

8. Validations
Determine structure of supervisory control layer with Gramian based interaction measures

\subsubsection{Degree of Freedom Analysis}

The process degrees of freedom can be divided into two catagories, dynamic and control degrees of freedom. Dynamic degrees of freedom (typically liquid levels of holdup tanks) are degrees of freedom that have no impact on steady state operation. The control degrees of freedom are defined as degrees of freedom that have an impact on steady state operation. Skogestad (Skogestad, 2004) divides the control degrees of freedom into two additional classes, optimization and steady-state degrees of freedom. Optimization degrees of freedom are defined as degrees of freedom that have an effect on the cost function defined in the first step and that 
will be used to optimize the process. Steady-state degrees of freedom are defined as degrees of freedom that effect the steady-state operation but not the operational objective. In general, however, the steady-state degrees of freedom (DOF) are the same as the optimization degrees of freedom. An example of a degree of freedom analysis from (Skogestad, 2004) is shown in Figure 2. This is an integrated distillation process with total DOF of 11. This is, for all intents, an exercise in 'valve counting' as, in general, the DOF's are available through the manipulation of the valves. Four of these DOF's are associated with liquid levels, and therefore are dynamic DOF's with no steady-state effect. Removing these four DOF's from the original eleven results in seven control DOF's that have steady-state effects.

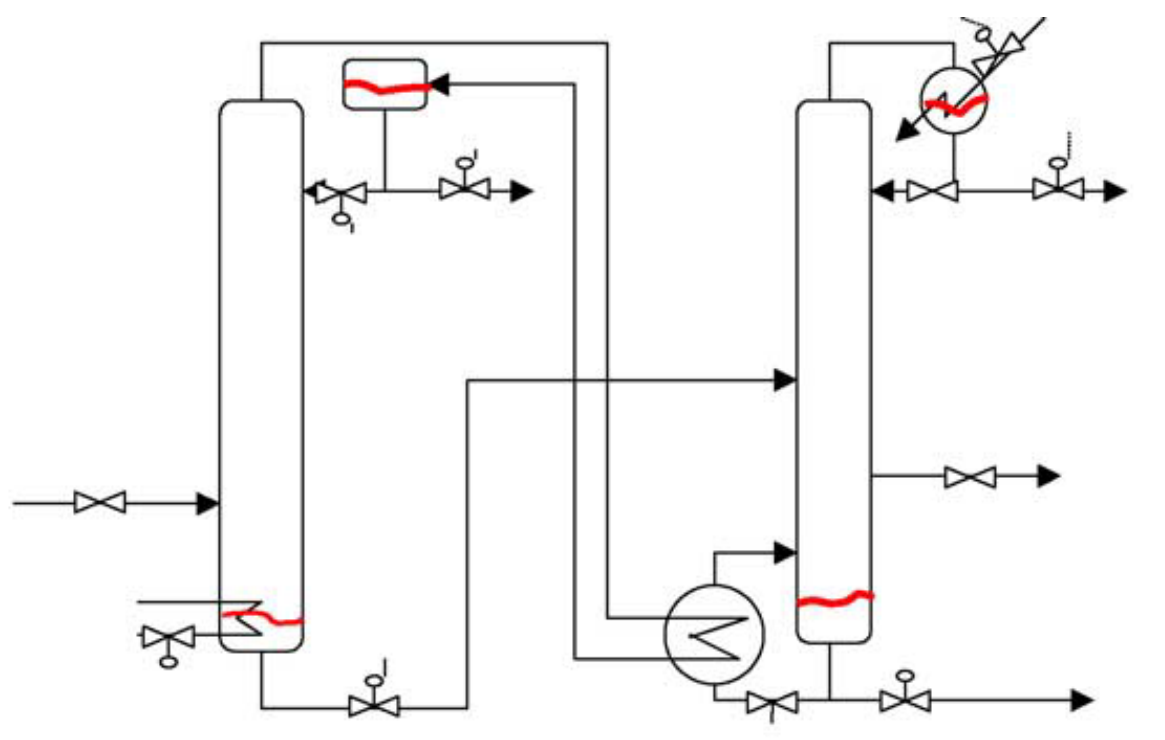

$$
\mathrm{N}_{\mathrm{m}}=11 \text { (incl. feed), } \mathrm{N}_{0 \mathrm{y}}=4 \text { (levels), } \mathrm{N}_{\mathrm{ss}}=11-4=7
$$

Figure 2: Example of degree of freedom analysis (Skogestad, 2004)

\subsubsection{Primary Controlled Variable Selection}

An important step in the design of a control structure is choosing what should be controlled. From the work of Skogestad, 2004 and Araujo et al, 2007, determining which controlled variables should be choosen is an involved, multistep process. First, several optimization studies must be undertaken. The process is optimized with respect to an operational objective at the nominal operation point using the degrees of freedom previously identified and subject to all process and enviornmental constraints. With this complete, likely disturbances that the process may encounter are identified. The process is then reoptimized under varying magnitudes of these identified disturbances. These disturbances may include changes in flowrate, 
compositions, temperatures, operational and environmental constraints among others possibilites. This reoptimization is undertaken by implementing the disturbance onto the model and then optimizing the process. Constraints that are active under all disturbances will be chosen as controlled variables (Maarleveld \& Rijnsdrop, 1970;Skogestad , 2000). Any degrees of freedom remaining after active constraints are controlled need additional controlled variables. It is desired that these remaining controlled variables will result in self-optimizing control. Selfoptimizing control variables, as defined by Skogestad, are those that result in acceptable loss when a constant setpoint policy is used. Here, loss is defined as the difference in the objective function values between the optimized cases and the constant setpoint cases. Morari et al (1980) described the self-optimizing controlled variables as those that "when held constant, lead automatically to the optimal adjustments of the manipulated variables." Figure 3, from (Skogestad, 2004), is a graphical representation that depicts the general idea of self-optimizing control variables. The controlled variable set $\mathrm{C}_{2, \mathrm{~s}}$ results in higher loss than $\mathrm{C}_{1, \mathrm{~s}}$. For this reason, $\mathrm{C}_{1, \mathrm{~s}}$ would be called a self-optimizing controlled variable set as compared to $\mathrm{C}_{2, \mathrm{~s}}$. It is important to note that self-optimizing performance is a relative term. For example, one controlled variable set has better self-optimizing performance as compared to another controlled variable set. 


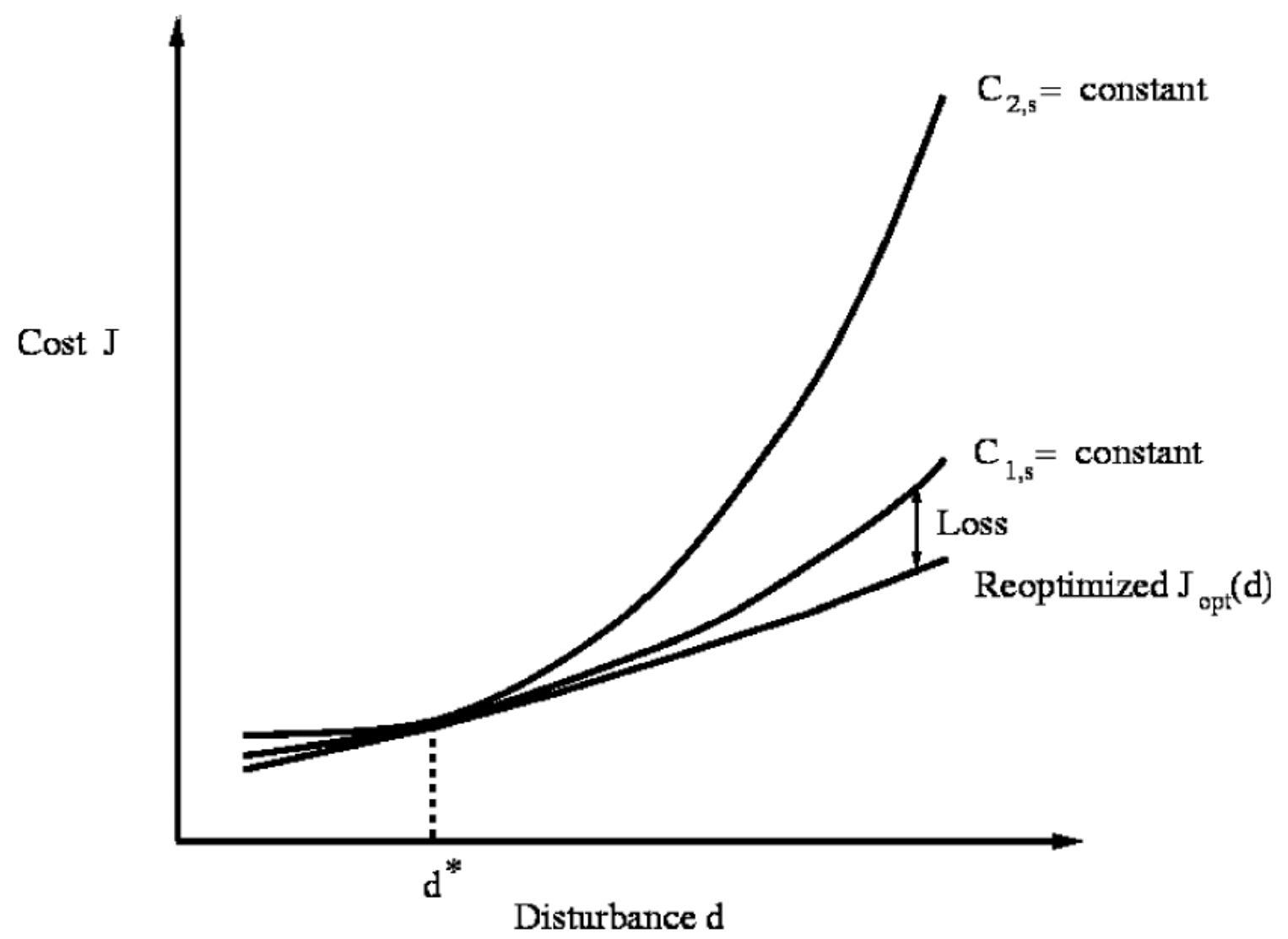

Figure 3: Comparison of constant setpoint policy with two controlled variables sets where $\mathrm{d}^{*}$ is the nominal operating point (Skogestad, 2004)

There are several proposed methods for finding these self-optimizing control variables: the local linear method (Skogestad , 2000), the exact local worst-case method (Halvorsen et al, 2003), and the local average loss method (Kariwala \& Cao, 2008). A major advantage of these methods is that they do not require any dynamic information, only steady state information. Each of these methods start by defining a loss function for the process. The loss functions for the local linear method, the exact local method, and the local average loss method are shown as Equations 1, 2, and 3, respectively. Equation 1 is based upon a Taylor series expansion of the loss function out to the $3^{\text {rd }}$ term. Equations 2 and 3 are exact solutions to the loss function. The basic concept behind Equations 2 and 3 is determining how disturbances will effect the primary controlled variables and how the input variables will have to be manipulated to maintain those controlled variables at their setpoints. Additionally, the effect of the disturbances on the objective function itself is considered, e.g., how changes in throughput of a plant effects the loss itself. Finally, how the disturbances and the manipulated variables work together to effect the loss function are 
brought together in the first term of Equations 2 and 3. Additionally, the effect of implementation error is also included in Equations 2 and 3. This term is, essentially, an additive term that accounts for any implementation error that will effect the cost function. It is important to note that the formulations of Equations 2 and 3 are the same, they differ only in respect to the matrix norm that is applied to them. Additionally, these selection criteria neither consider the controllability nor the observability of the resulting process.

$$
\begin{aligned}
& L_{\max }=\frac{\underline{\sigma}\left(J_{u u}\right)}{2 \underline{\sigma}(S G)^{2}} \\
& L_{\text {worst }}=\frac{1}{2} \bar{\sigma}^{2}\left(\left[\begin{array}{cc}
J_{u u}^{\frac{1}{2}}\left(J_{u u}^{-1} J_{u d}-G^{-1} G_{d}\right) W_{d} & J_{u u}^{\frac{1}{2}} G^{-1} W_{e}
\end{array}\right]\right) \\
& L_{\text {average }}=\frac{1}{6\left(n_{y}+n_{d}\right)}\left\|\left[\left(G J_{u u}^{-\frac{1}{2}}\right)^{-1}\left[\left(G J_{u u}^{-1} J_{u d}-G_{d}\right) W_{d} \quad W_{e}\right]\right]\right\|_{F}^{2}
\end{aligned}
$$

The above methods all assume the linear model shown as Equation 4. Loss is defined as Equation 5.

$$
\begin{gathered}
y=G u+G_{d} d+W_{e} e \\
L=\left.J(u, d, e)\right|_{c=c^{\text {nom }}}-J\left(u^{o p t}(d), d\right)
\end{gathered}
$$

where $J$ is the cost function, $G$ is the steady-state gain matrix, $G_{d}$ is the disturbance gain matrix, $J_{u u}$ is $\frac{\partial^{2} J}{\partial u^{2}}, J_{u d}$ is $\frac{\partial^{2} J}{\partial u \partial d}, n_{y}$ is the number of controlled variables to be selected, $n_{d}$ is the number of disturbances considered, and $S, W_{d}$, and $W_{e}$ are all scaling matrices. The scaling matrix $S$ is based on the range of the candidate controlled variables from the optimization studies. Specifically, $S=\operatorname{diag}\left\{\frac{1}{\operatorname{span}\left(c_{i}\right)}\right\}$ where $\operatorname{span}\left(c_{i}\right)=\max \left|c_{i}^{n o m}-c_{i}^{d}\right|$ with $c_{i}^{\text {nom }}$ as the value of the candidate controlled variable $i$ at nominal operation and $c_{i}^{d}$ is the value of the candidate controlled variable due to the disturbance $d$. The matrices $W_{d}$ and $W_{e}$ are scaling matrices used so that the magnitudes of the disturbance and implementation error vectors are between 0 and 1 . The way in which these equations are used to find candidate self-optimizing control variables is the same. Note that all of the variables in these equations are independent of the controlled variables with the exception of the steady-state gain matrix and the disturbance gain matrix. 
Because of this, candidate controlled variables can be compared by the evaluation of these equations with the respective controlled variables included in the gain matrices while the others are removed. These relations are advantageous compared to direct evaluation of the loss function using the non-linear process model in several important respects. First, these relations are far less computationally expensive. Second, the gain matrix, which is the only variable in these equations that is dependent upon the selected controlled variables, can be calculated beforehand. The gain matrix used in any of the above equations is composed of selected rows of the original gain matrices containing all candidate controlled variables. Therefore, by appending the gain matrices used in these equations from the elements of the gain matrices calculated from the process model, the comparison of a large number of controlled variable sets is significantly simpler and computationally less expensive.

It is important to note, however, that although these equations allow for an easier and computationally less expensive method for the evaluation of loss, a method for determing the globablly optimal set of controlled variables is required. For example, consider a system (the Selexol ${ }^{\mathrm{TM}}$ process) that has 320 possible controlled variables and 6 degrees of freedom. This system has over 1.4 trillion possible controlled variable sets. Evaluation of all of these possibilites is impossible even with the simplified methods of evaluation of loss. Consider the first equation, the local linear loss, the least compuationally expensive function. Evaluation of this function for a system of the size described above takes approximately $0.05 \mathrm{~ms}$. Therefore, evaluating all of these possibilities would take over 2.2 years. Additionally, there is no method for determining which controlled variables should even be considered in the first place. The method most commonly used for solving these types of problems is a branch and bound algorithm (Araujo et al, 2007; Cao \& Kariwala, 2008; Kariwala \& Cao, 2009; Kariwala \& Cao, 2010). The branch and bound algorithm is well suited for this particular problem because the objective functions are, or can be made to be, applicable for branch and bound algorithm . Additionally, branch and bound is unique in that it is the only method, with the exception of brute force enumeration, that can guarentee a globablly optimal solution (Chen, 2003).

When a list of controlled variable sets is generated, direct evalution of loss and feasibility is undertaken using the nonlinear process model. These sets are evaluated at all of the disturbances that have been considred. Generally, the set which yields the lowest average loss across all the disturbances considered is selected as the primary controlled variable set. Skogestad (Skogestad, 2000) includes additional requirements on the selected controlled variables, in addtion to 
minimizing loss. First, the controlled variables should be easy to measure so as to minimize implementation error. Second, the controlled variables should be sensitive to changes in the inputs. Third, the selected controlled variables should be independent of one another when more than one needs to be selected. It is claimed by Skogestad that the last two criteria should automatically be satisfied under most conditions since the gain matrix includes this information. The claim is also based upon the assumption that after active constraints are controlled, few remaining controlled variables will be needed. However, no work can be found within the open literature that investigates and compares the controllability of the controlled variable sets. Due to this, it is difficult to determine whether acceptable control preformance from the controlled variable set that is chosen can be attained or not. Essentially, the problem is that although the selected controlled variable set may provide the best steady state preformance, the dynamics could be such that steady state may not be realizable.

\subsection{Bottom-Up Design}

During the bottom-up design section of the design procedure, the control structure is determined, secondary controlled variables are selected, and the controllers for the system are designed.

\subsubsection{Secondary Controlled Variable Selection}

In a plant-wide control system design procedure, the selection of controlled variables for the regulatory control layer is an important consideration. As discussed by Skogestad (2004), the control layers are generally divided into the upper supervisory controls and the lower regulatory control layers. Supervisory controls are used for the control of the primary controlled variables, associated with the economics of the process, and the regulatory controls are required to stabilize the plant by controlling the secondary controlled variables. Selection of a secondary controlled variable is driven primarily by how well it indirectly controls performance of the primary controlled variables, thereby enabling it to be used as a degree of freedom by the upper layers. Additional important considerations for secondary controlled variable selection are ease of measurement, sensitivity to the input variables, and minimal loop interactions.

As the secondary controlled variables are controlled on a faster time scale, the regulatory control layer should be as simple as possible (i.e., consisting mainly of PID controllers). Traditionally the determination of the indirect control performance of the controlled variable set is determined by a partially controlled plant analysis (Shinnar, 1981; Kothare et al., 2000; Luyben et al., 1998; Konda et al., 2005). A block flow diagram of a partially controlled plant analysis is shown in 
Figure 4. Here, $y_{1}$ and $y_{2}$ are the primary and secondary controlled variables, respectively. From this block flow diagram, it can be shown that, when $K_{2}$ approaches infinity, the primary controlled variables are given, as reported by Skogestad and Postlethwite (2005), as $y_{1}=\left(G_{11}-G_{12} G_{22}^{-1} G_{21}\right) u_{1}+\left(G_{d_{1}}-G_{12} G_{22}^{-1} G_{d_{2}}\right) d+G_{12} G_{22}^{-1}\left(\left(y_{2}\right)_{r e f}-n_{2}\right)$. Here, $G_{u y}$ is the process gain matrix from input $u$ to output $y$ and $G_{d 1}$ and $G_{d 2}$ are the disturbance gain matrices for the primary and secondary controlled variables, respectively. From this expression it is possible to derive several measures of control performance. However, a limitation of this partially controlled plant analysis is that it is a steady-state evaluation and does not address the dynamics of the system. In addition, two of the important considerations when selecting secondary controlled variables are ease of measurement and controllability and a purely steady-state based analysis cannot address these issues.

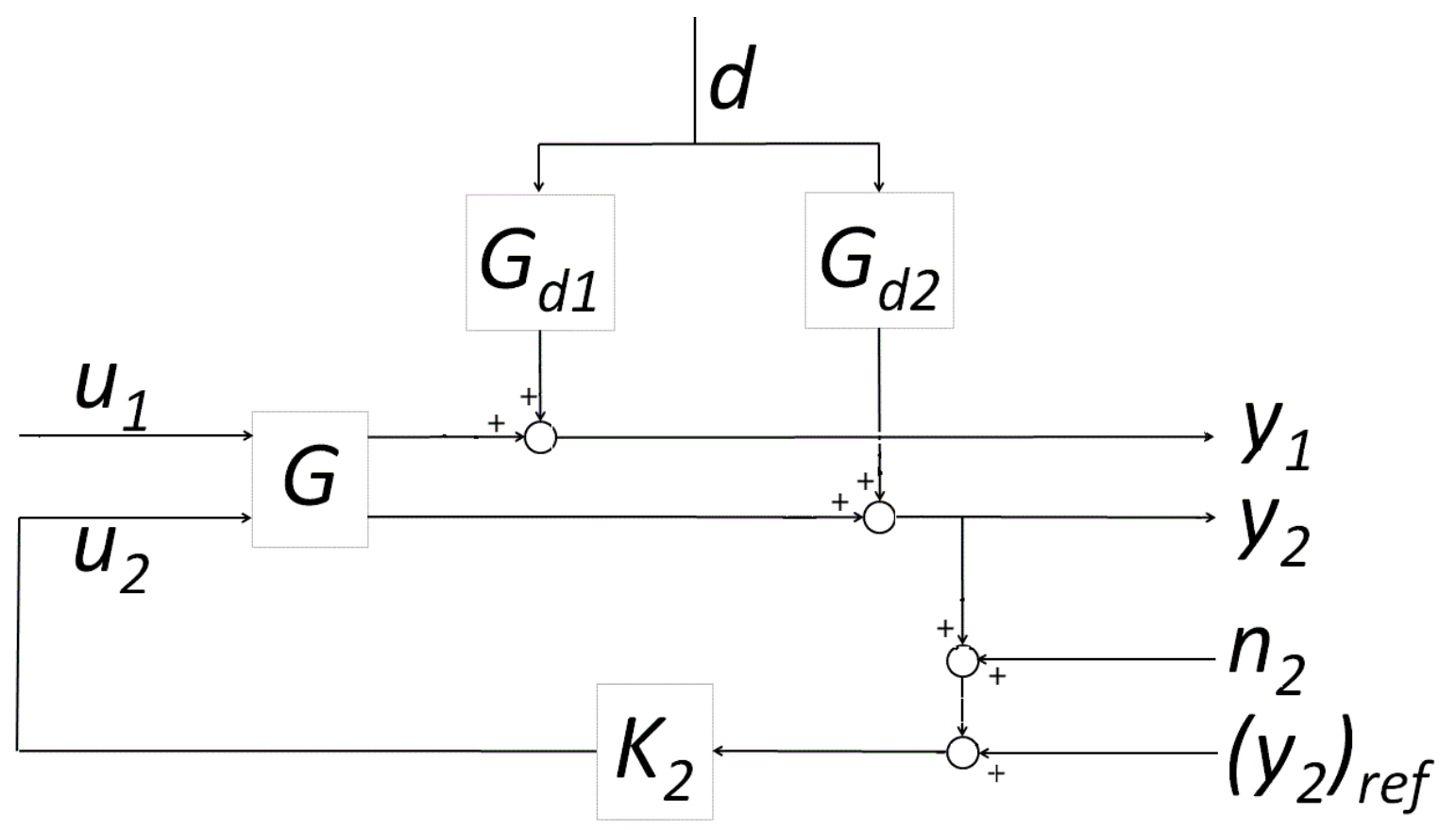

Figure 4: Partially Control Plant Block Flow Diagram

A new method for secondary controlled variables selection has recently been published by Yelchuru and Skogestad [2013]. In this method, the objective is the selection of a set of secondary controlled variables or a linear combination of secondary controlled variables that minimizes the 'state drift' of the plant. This method of secondary controlled variable selection, as applied by the authors, was limited to only steady-state analysis. However, the method itself could be applied to dynamic systems. This method also neither accounts directly for the servo or 
regulatory control performance of the supervisory control layer, which can be drastically affected by the secondary controlled variables. Another method for system stabilization is proposed by Alonso and Ydstie [2001] which connects thermodynamics and the passivity theory of control. This method, however, does not consider the control performance of a primary control loop. Although the process may be stabilized, the economic/primary controlled variable performance may not be satisfactory.

\subsubsection{Interaction Analysis}

Four major interaction measures are available within the open literature. These are the relative gain array (RGA) analysis (Bristol, 1966), the Participation Matrices (PM) (Conley \& Salgado, 2000), the Hankel Interaction Index Array (HIIA) (Wittenmark \& Salgado, 2002), and the $\Sigma_{2}$ measure (Birk \& Medvedev, 2003). The RGA is given by Equation 6 where $G$ is the steady-state gain and '.*' denotes element by element matrix multiplication. The RGA is, essentially, a normalized gain matrix that allows for easier determination of appropriate input-output pairings for multi-input-multi-output (MIMO) systems. Consider an arbitrary RGA shown as Equation 7. Here, the element $\lambda_{i j}$ corresponds to $y_{i}$ and $u_{j}$. Equation 8 is the formal definition of what the elements of the RGA represent. Each of these elements shows how the gain of input $j$ on output $i$ changes when all remaining loops are closed. Therefore, $\lambda_{i j}>1$ indicates that the gain of input $j$ on output $i$ will decrease when all remaining control loops are closed. Likewise, when $\lambda_{i j}<1$ indicates that the gain of input $j$ on output $i$ will increase when all other control loops are closed. Finally, if $\lambda_{i j}<0$ indicates that the gain of input $j$ on output $i$ has changed direction, that is changes in $j$ will have the opposite effect on $i$ when all other control loops are closed. This provides information on loop-loop interactions as the further away an element is from 1, the higher the degree of loop-loop interactions. Finally, the RGA has several significant properties. The RGA is independent of the scaling applied to $G$ (Halvarsson, 2010). Additionally, the

numerical sum of any row or column is equal to 1 . To demonstrate how pairing is accomplished using the RGA, consider a $2 \times 2$ system whose RGA is given by Equation 9 .

$$
\Lambda(G)=G(0) \cdot *\left(G(0)^{-1}\right)^{\mathrm{T}}
$$




$$
\begin{aligned}
R G A=\Lambda(G) & =\left[\begin{array}{cccc}
\lambda_{11} & \lambda_{12} & \cdots & \lambda_{1 n} \\
\lambda_{21} & \lambda_{22} & \cdots & \lambda_{2 n} \\
\vdots & \vdots & \ddots & \vdots \\
\lambda_{n 1} & \lambda_{n 2} & \cdots & \lambda_{n n}
\end{array}\right] \\
\lambda_{i j} & =\frac{\left(\frac{\partial y_{i}}{\partial u_{j}}\right)_{\text {all loops open }}}{\left(\frac{\partial y_{i}}{\partial u_{j}}\right)_{\text {only loop } i \text { open }}} \\
\Lambda(G) & =\left[\begin{array}{cc}
\lambda & \lambda-1 \\
\lambda-1 & \lambda
\end{array}\right]
\end{aligned}
$$

(Kinnaert, 1995) shows 5 possible cases. If $\lambda=1$, this is the ideal case, where no interaction exists and the pairing should be $y_{1}-u_{1}$ and $y_{2}-u_{2}$. If $\lambda=0$, this, like the first case, is the ideal case, except the pairing should be $y_{1}-u_{2}$ and $y_{2}-u_{1}$. If $0<\lambda<1$, the gain will increase when the loops are closed, where $\lambda=0.5$ represents the worst-case, i.e., most interaction. If $\lambda>1$, the gain decreases when the loops are closed, where interaction increases as $\lambda$ increases. If $\lambda<0$, the sign of the gain changes when the loops are closed. This is highly undesired and should be avoided. Interaction increases as $\lambda$ becomes more negative. In short, pairing should be done such that the resulting RGA is as close as possible to the identity matrix.

The remaining three interaction measures all rely upon the controllability and observability Gramians. Consider the following continuous time-invariant state-space model:

$$
\begin{aligned}
& \dot{x}(t)=A x(t)+B u(t) \\
& y(t)=C x(t)
\end{aligned}
$$

where $x(t)$ is the state vector, $u(t)$ is the input vector, and $y(t)$ is the output vector. The controllability and observability Gramians for this system are defined by Equations 11 and 12, respectively. Additionally, the controllability and observability Gramians may be obtained by solving the Lyapunov equations, Equations 13 and 14, respectively. The controllability Gramian is a measure of the effect of the inputs on the states across the entire frequency range of the system. 


$$
\begin{gathered}
P=\int_{0}^{\infty} e^{A \tau} B B^{\mathrm{T}} e^{A^{\mathrm{T}} \tau} d \tau \\
Q=\int_{0}^{\infty} e^{A^{\mathrm{T}} \tau} C^{\mathrm{T}} C e^{A \tau} d \tau \\
A P+P A^{\mathrm{T}}+B B^{\mathrm{T}}=0 \\
A^{\mathrm{T}} Q+Q A+C^{\mathrm{T}} C=0
\end{gathered}
$$

All of the Gramian based interaction measures work in essentially the same way. The controllability Gramian is a measure of the influence that each manipulated variable has on the states of the process. The observability Gramian is a measure of the influence of the states on the outputs. The product of these two Gramians provides information as to the influence that an input has on an output. The difference between all the Gramian based interaction measures is only in terms of which matrix norm is applied to the resulting matrix. These interaction measures are superior to the RGA as they examine the process across the entire frequency range of the process as well as making no assumption of how the control structure will be. Specifically, the Gramian based methods do not assume that a decentralized control structure will be used.

\subsubsection{HIIA}

The HIIA (Wittenmark \& Salgado, 2002) involves breaking a multi-input multi-output (MIMO) system represented as $(A, B, C, O)$ into multiple single-input single-output (SISO) subsystems represented as $\left(A, B_{* ;}, C_{i^{*}}, O\right)$. The subsystem is based upon the single input, $j$, and the single output, $i$. This subsystem is generated by taking the $j^{\text {th }}$ column of $B$ and the $i^{\text {th }}$ row of $C$ as the SISO system. Controllability and observability matrices for this SISO system, $P_{j}$ and $Q_{i}$ respectively, can then be calculated. To generate the HIIA matrix, all possible SISO subsystems must be generated, i.e., all possible pairings of inputs with outputs. From these SISO subsystems, the Hankel norms, $\|\bullet\|_{\mathrm{H}}$, of the product of the controllability Gramian with the observability Gramian must be calculated. The Hankel norm is defined in Equation 15. When this is completed, the HIIA matrix is given by Equation 16. 


$$
\begin{array}{r}
\|G\|_{\mathrm{H}}=\sqrt{\lambda_{\text {max }}(G)} \\
{\left[\Sigma_{H}\right]_{i j}=\frac{\left\|P_{i} Q_{j}\right\|_{\mathrm{H}}}{\sum_{k l}\left\|P_{k} Q_{l}\right\|_{\mathrm{H}}}}
\end{array}
$$

$\Sigma_{H}$ denotes the HIIA, $P_{j}$ and $Q_{i}$ are the controllability and observability Gramians of the SISO subsystem, respectively. Note that the HIIA matrix is scaled in such a way that the sum of all of its elements will equal 1. How the HIIA analysis and the other Gramian interaction measures are used for determining control variable pairings as well how they assist in determining the optimal structure of the control system will be discussed in section 2.2.3.4.

\subsubsection{PM}

The PM interaction measure (Conley \& Salgado, 2000) is very similar to the HIIA interaction. An argument against the HIIA analysis is that it considers only the largest Hankel singular value, but not all of them (Halvarsson, 2010). This can be a problem if several Hankel singular values are of the same order of magnitude. The difference between the HIIA and the PM is that the PM considers all of the Hankel singular values. Equation 17 is used for generating the PM.

$$
[\Phi]_{i j}=\frac{\operatorname{tr}\left(P_{j} Q_{i}\right)}{\operatorname{tr}(P Q)}
$$

$\Phi$ denotes the PM, $\operatorname{tr}$ denotes the trace, $P_{j}$ and $Q_{i}$ are the controllability and observability Gramians of the SISO subsystem respectively, and $P$ and $Q$ are the controllability and observability Gramians of the entire MIMO system. The PM is scaled in this way so that, as with the HIIA, the sum of all elements equals 1.

\subsubsection{3. $\Sigma_{2}$ Interaction Analysis}

The $\Sigma_{2}$ interaction measure (Birk \& Medvedev, 2003) is nearly identical to the HIIA except the Hankel norm is exchanged with the $\mathrm{H}_{2}$ norm (Skogestad \& Postlethwaite, 1996). The $\mathrm{H}_{2}$ norm is defined in Equation 18. However, the work of (Halvarsson, 2010) shows that the calculation of the $\mathrm{H}_{2}$ norm can be simplified for a state-space model $(A, B, C, O)$ using Equation 19. Equation 20 is used to generate the $\Sigma_{2}$ matrix. Essentially, the $\Sigma_{2}$ interaction measure, like the PM interaction measure, takes into account the contribution of all states, as indicated by taking a 
trace. The difference between the $\Sigma_{2}$ from the PM is the use of the output controllability Gramian rather than the product of the controllability and observability Gramians

$$
\begin{gathered}
\|G(s)\|_{2} \equiv \sqrt{\sum_{i, j} \int_{0}^{\infty}\left|g_{i j}(\tau)\right|^{2} d \tau} \\
\|G(s)\|_{2} \equiv \sqrt{\operatorname{tr}\left(B^{\mathrm{T}} Q B\right)}=\sqrt{\operatorname{tr}\left(C P C^{\mathrm{T}}\right)} \\
{\left[\Sigma_{2}\right]_{i j}=\frac{\left\|P_{j} Q_{i}\right\|_{2}}{\sum_{k l}\left\|P_{k} Q_{l}\right\|_{2}}}
\end{gathered}
$$

Note, like all other Gramian based interaction measures, that the $\Sigma_{2}$ matrix is scaled in such a way that the sum of all elements equals 1 .

\subsubsection{Supervisory Control Layer}

Before the controller(s) of the supervisory control layer are designed, the structure of the control structure must be determined. For a simple process with little interaction between control loops, decentralized, PID control can be used. If there is a high degree of loop interactions, one may choose a model predictive controller (MPC) for the process. In this section, how the Gramian interaction measures can be used to determine the optimal structure of the supervisory control layer.

\subsubsection{Control Structure Design}

To show how paring is accomplished with the Gramian based interaction measure and how it can be used to determine the control structure to be selected, consider this $3 \times 3$ example from (Halvarsson, 2010):

$$
\begin{array}{ccc}
\Sigma_{H}=\left[\begin{array}{lll}
0.0703 & 0.1663 & 0.0728 \\
0.1728 & 0.0878 & 0.0728 \\
0.1426 & 0.0781 & 0.1367
\end{array}\right] & \Lambda(G(0))=\left[\begin{array}{ccc}
-0.0831 & 0.9111 & 0.1720 \\
1.3809 & -0.2745 & -0.1064 \\
-0.2979 & 0.3634 & 0.9345
\end{array}\right] \\
\Phi & =\left[\begin{array}{lll}
0.0370 & 0.2018 & 0.0385 \\
0.2226 & 0.0578 & 0.0385 \\
0.2193 & 0.0457 & 0.1389
\end{array}\right] & \Sigma_{2}=\left[\begin{array}{ccc}
0.0316 & 0.2331 & 0.1119 \\
0.0913 & 0.0559 & 0.0791 \\
0.2292 & 0.0609 & 0.1070
\end{array}\right]
\end{array}
$$


$\Lambda(\mathrm{G}(0))$ denotes the RGA. The goal when using any of the Gramian based methods is to select elements that will result in a maximized sum. Obviously, for decentralized control, only one element from each row and column may be selected. Therefore, for a decentralized control design, the pairings $y_{1}-u_{2}, y_{2}-u_{1}$, and $y_{3}-u_{3}$ are suggested by the RGA, HIIA, and PC. The $\Sigma_{2}$ interaction measure however suggests the pairings $y_{1}-u_{2}, y_{2}-u_{3}$, and $y_{3}-u_{1}$. The RGA analysis, however, would suggest that these pairing would result in instability and should be avoided.

The Gramian based methods are superior to RGA as they can suggest control structures in addition to decentralized control and their applicability is not limited to decentralized control. The method is the same for all the Gramian based methods so, for simplicity, the HIIA will be used to demonstrate the method. The sum of elements that is attained with the decentralized control is 0.4758; however, if the element $(3,1)$ is also included (the largest element not currently included), the sum is 0.6184 . The inclusion of this element results in a control structure in which $y_{3}$ is 'controlled' by both $u_{1}$ and $u_{3}$. More accurately, $u_{1}$ would be an input to a feed-forward controller that is part of a feed-forward augmented feedback control strategy for controlling $y_{3}$ by manipulating $u_{3}$. This is still a relatively simple control structure that requires no centralized control; however, the HIIA analysis suggests a significant improvement in performance as compared to decentralized control. If the next largest element $(2,2)$ is included, the resulting sum is 0.7062 . The inclusion of the element is equivalent to the addition of a feedforward controller which accounts for the effect of $u_{2}$ on $y_{2}$ to the previous control system. Again, this does not significantly increase the complexity of the control structure as still no centralized controller is added.

Continuing the procedure, we can add the next largest element $(3,2)$ to further improve the control performance. With the addition of element $(3,2)$, a centralized controller is now required to control the outputs $y_{2}$ and $y_{3}$ with the inputs $u_{1}$ and $u_{3}$. Note, however, that this does not substantially increase the sum of elements. The previously suggested design, which included one feedback controller as well as two feed-forward augmented feedback controllers, had a sum of 0.7062. The current suggestion consists of a centralized controller and a feed-back controller and yields a sum of 0.7843 .

This process can continue until all elements are included, resulting in a completely centralized control strategy. It is important to note that with each addition, controller complexity increases 
with increasingly small improvements in control performance. Ultimately, the Gramian based methods can help inform a decision on the control structure, but it does not give a definitive answer to which structure provides the best balance of performance against complexity. In addition, unlike the RGA, the Gramian based methods do not yield any information about how controllable the resulting process will be.

\subsubsection{Validation}

Finally, the proposed control structure must be validated using the nonlinear dynamic model. 


\section{Contributions}

In this section, the contributions of this work to the field of plantwide control will be discussed. Major contributions during the course of this work are in the area of primary controlled variable selection, secondary controlled variable selection, and control structure design.

\subsection{Primary Controlled Variable Selection}

During this work it has been found that considering only the economic performance of the primary controlled variables, as currently done within the open literature, will yield uncontrollable and/or infeasible controlled variables. This can be due to the lack of manipulated variables with the necessary gain to control the primary controlled variables and/or a high degree of interaction between the primary controlled variables that will result in a high degree of loop interactions. Finally, the 'distance' of the manipulated variable from the controlled variable may be such that the associated dead-time with that control loop is very large, resulting in poor control performance. The method for primary controlled variables selection proposed in this paper addresses these issues. This method involves a three-stage procedure: a priori analysis, controlled variable selection, and a posteriori analysis. The contributions of this work to each of these stages are summarized below.

- A Priori Analysis

- Prescreening criteria have been added to the control structure design procedure to help eliminate infeasible controlled variable sets from consideration and to reduce the size of the large scale combinatorial optimization problem. The prescreening criteria identify controlled variables that would show either poor servo or regulatory control performance and eliminates them from further consideration. This allows for exploration of the feasible controlled variables by eliminating the infeasible controlled variables at the start of the selection process.

- Controlled Variable Selection

- A controllability measure has been added within the framework of the primary controlled variable selection problem. It has been found during the course of this work that primary controlled variables selected solely upon their economic performance will lead to infeasible and/or uncontrollable controlled variable 
sets. The controllability measure and additional constraints as mentioned below are included in the algorithm to take care of this issue.

- Loop interactions are included within the framework as a controlled variable set may show good economic and control performance, but fail to attain satisfactory control performance due to strong loop interactions.

- A constraint is added to the selection methodology to address the issue of poor control performance for time-delay systems. The constraint is formulated by considering the dead-time of the paired manipulated variable with the controlled variable.

- A Posteriori Analysis

- In addition to the evaluation of the economic performance of the selected controlled variables by using the nonlinear model, control performance is also evaluated by using the nonlinear model. Examination is undertaken at offdesign operations considering the presence of a real-time optimizer (RTO) (process is at the optimal operational point) and the absence of an RTO (primary controlled variables are left constant at their nominal values).

\subsubsection{A Priori Analysis}

The a priori analysis begins by defining an operational objective that is to be optimized. Operational and other constraints are then identified along with likely disturbances to which the process may be subjected. The process is then optimized with respect to this operational objective at the nominal operating point as well as under the identified disturbances. From these optimization studies, active constraints are identified (Skogestad, 2004). However, further analysis is required to determine appropriate pairings of manipulated variables with active constraints as well as identification of a candidate set of controlled variables for controlled variables analysis.

\subsubsection{Optimization}

The process of selecting primary controlled variables begins with the definition of an operational objective that is to be optimized. This is followed by a degree of freedom analysis to identify the manipulated variables available for the control of the system. This is preceded by the identification of process constraints that can be operational or environmental constraints. Finally, the disturbances likely to affect the process must be determined. At this point, the 
process is optimized in relation to the operational objective using the identified degrees of freedom and subject to the identified constraints. This optimization study is completed at the nominal operating point of the process and also under disturbance conditions. These optimization studies yield important information related to the optimal variations of the input and output variables as well as optimal operations at these conditions. The identification of the optimal operational regions is important for later stage analysis of controllability of the controlled variable sets as well as for the actual design of the supervisory control layer. The optimal variations are used in later stage analyses as scaling matrices defined in Equations 21 and 22, where subscripts $i$ and $j$ denote outputs and inputs respectively and nom and $d$ denote their nominal operation and disturbance operations. These are similar scaling factors as proposed by (Skogestad and Postlethwaite, 2005). Finally, the optimization studies identify the active constraints of the process. Active constraints are defined as those constraints that are active at all operations investigated.

$$
\begin{gathered}
D_{\text {output }}=\operatorname{diag}\left\{\left(\operatorname{span}\left(c_{i}\right)\right)^{-1}\right\} \mathrm{w} / \operatorname{span}\left(c_{i}\right)=\max \left|c_{i}^{\text {nom }}-c_{i}^{d}\right| \\
D_{\text {input }}=\operatorname{diag}\left\{\operatorname{span}\left(u_{j}\right)\right\} \mathrm{w} / \operatorname{span}\left(u_{j}\right)=\max \left|u_{j}^{\text {nom }}-u_{j}^{d}\right|
\end{gathered}
$$

\subsubsection{Control of Active Constraints}

For the process to be operated efficiently, the active constraints identified during the optimization studies must be controlled (Skogestad, 2000; Maarleveld \& Rijnsdrop, 1970). Since the bounds on the variability of the active constraints are tighter than any other controlled variables, the assumption taken in this methodology is that the control performance of the active constraints must be the highest priority.

The measure taken for the quantification of the control performance of the active constraint controls is the RGA number, defined in Equation 23 where $\Lambda(G)$ is the relative gain array. The RGA number is a measure of the loop interaction of the resulting closed-loop system. The objective is the selection of manipulated variables for the control of the active constraints which will result in a minimal RGA number, therefore minimal loop interaction. Additionally, this provides information about the least amount of closed-loop interactions possible for the control of the active constraints. The RGA number does not determine whether the manipulated variable has the required gain to control the active constraint. Additionally, the RGA number does not address the associated dead-time of the manipulated variable's effect on the active constraint. 
For this reason, the manipulated variables should not necessarily be selected for the global minimal RGA number. The selection of the manipulated variables should also consider these important issues.

$$
\text { RGA number }=\|\Lambda(G)-I\|_{S U M}
$$

\subsubsection{Identification of the Candidate Sets of Controlled Variables}

The first step of the procedure is the generation of a set of candidate controlled variables. Very little work can be found in the existing literature for generating the candidate controlled variable sets (Alonso \& Ydstie, 2001). Our proposed method is described below.

Obviously, one would like to consider all possible controlled variables: algebraic and differential process variables, e.g., temperatures, compositions, flows, and derived variables, e.g., ratios of flows and compositions, in this set. However, not all candidate controlled variables are, in and of themselves, controllable. In addition, a large candidate controlled variable set will greatly increase the number of possible sets. For small scale systems, this may not be an issue as candidate controlled variables that many be uncontrollable can be identified and discarded. Additionally, as the number of manipulated variables in smaller processes is expected to be less, the number of possible controlled variable sets will be less even if the number of candidate controlled variables considered is the same. Therefore, the consideration of more candidate controlled variables may remain computationally tractable for smaller systems, whereas in larger systems it may become intractable.

For the generation of a candidate controlled variable set, an initial set of controlled variables is prepared. The initial set of controlled variables contains those variables that the user suspects are valid controlled variables for the process. The generation of this initial set is dependent upon the process insight of the user. The candidate set will be generated from the initial set defined by the user and it is ultimately the candidate set that will be used in later stage analyses. For determining the candidate set from the initial set, the linear process model, defined as Equation 24 , is calculated with the active constraint controls in place for all of the candidate controlled variables. $G_{p}$ is the process gain matrix and $G_{d}$ is the disturbance gain matrix. These gain matrices will then be scaled in such a way that elements of the vectors $y, u$, and $d$ are all of magnitude 1 or less. With the process and disturbance gain matrices scaled in this manner, all candidate controlled variables are evaluated and checked against the criteria in Equations 25 and 26. If both of these equations are not satisfied, the corresponding candidate controlled variable is 
not considered for further analysis. Equations 25 and 26 are measures of the servo and regulatory control performance, respectively. From the linear process model, it is obvious that if Equations 25 and 26 are not true and the process model is scaled appropriately, that no input variable exists that can control output variable $i$ subject to inputs and outputs magnitudes being bound to less than 1. This method of generating a set of candidate controlled variables can reduce the number of possible candidate controlled variable sets to a great extent, allowing for the examination of a larger number of initial sets as expected in large-scale plants. It is important to note that within the initial candidate set, the manipulated variable itself must be included. By including the manipulated variable itself within the initial candidate controlled variable set, it allows for the possibility of 'self-selection'. In this way, manipulated variables do not necessarily need to be paired with a controlled variable but instead may self-select, specifically, the manipulated variable is left at a fixed value. For example, consider an absorption column where the solvent flowrate is a degree of freedom that could be used for control. If the solvent flowrate is not used for control of another variable, it is said that it has 'self-selected', i.e., it is itself a controlled variable.

$$
\begin{gathered}
y=G_{p} u+G_{d} d \\
\left\|\left(G_{p}\right)_{i}\right\|_{M A X} \geq 1 \\
\left\|\left(G_{p}\right)_{i}\right\|_{M A X} \geq\left\|\left(G_{d}\right)_{i}\right\|_{M A X}
\end{gathered}
$$

\subsubsection{Selection of the Pareto-Optimal Controlled Variable Sets}

At this stage, a linearized process model is used to analyze the candidate controlled variable sets, identified in the previous stage, to determine their economic and control performance. In addition, the closed-loop interactions are also examined by a relative gain array (RGA) analysis. The Pareto-optimal controlled variable sets are further analyzed during the a posteriori analysis for obtaining the final set of primary controlled variables.

\subsubsection{Formulation of the Optimization Problem}

A constrained, multi-objective optimization problem is formulated for the generation of a set of primary controlled variables. The first objective is an economic objective. Two measures can be considered for determining self-optimizing performance of the controlled variable sets that directly consider the cost function: the local worst-case loss derived by (Halvorsen et al., 2003) and the local average loss derived by (Kariwala and Cao, 2010). In this work, we have 
considered the local average loss (Kariwala and Cao, 2010), Equation 27, for evaluating the selfoptimizing performance. $J$ is the cost function and $W_{d}$ and $W_{e}$ are diagonal matrices containing the expected magnitudes of the disturbances and implementation errors, respectively. Loss, defined in Equation 28, refers to economic loss incurred due to a constant setpoint policy. It should be noted that these measures of economic performance are very similar to one another. Both rely upon a partially controlled plant analyses, first proposed by (Shinnar 1981), which is scaled in relation to the economics. The difference between the two is in the matrix norm that is applied. Readers interested in a more detailed discussion of the use of partial control in the area of control structure design are directed to (Kothare et al., 2000). It should be noted that if large magnitudes of the disturbances are considered, i.e., large diagonal elements in $W_{d}$, the resulting matrix can become near singular and evaluation of the average loss function becomes meaningless. If this is the case, smaller magnitudes of the disturbances should be considered while ensuring that the relative magnitudes of all the disturbances are consistent with the anticipated magnitudes. This will change the absolute value of the function, but not its optimal value. Readers interested in the derivation of the local worst-case loss and the local average loss are directed to (Halvorsen et al., 2003) and (Kariwala and Cao, 2010).

$$
\begin{gathered}
L_{\text {average }}=\frac{1}{6\left(n_{y}+n_{d}\right)} \|\left[\left(G_{p} J_{u u}^{-\frac{1}{2}}\right)^{-1}\left[\left(G_{p} J_{u u}^{-1} J_{u d}-G_{d}\right) W_{d} W_{e}\right]\|\|_{F}^{2}\right. \\
L=J(c, d)-J_{\text {opt }}(d)
\end{gathered}
$$

The second objective is the controllability. The controllability measure selected in this work is the minimum singular value of the steady-state gain matrix. The singular value analysis was selected for the controllability measure as the singular values provide better information about the gain of the plant than the eigenvalues do (Skogestad \& Postlethwaite, 2005). The minimum singular value, Equation 29, was selected as the controllability measure as it represents the smallest gain for any input direction. In this equation, $P_{n}$ is a vector of logical 1's and 0's, denoting row selections. In addition, using the minimum singular value as a measure of the control performance is advantageous as it is not dependent upon the disturbances considered.

$$
\begin{aligned}
& J_{c}\left(P_{n}\right)=\underline{\sigma}^{-1}(\hat{G}) \\
& \hat{G}=D_{\text {output }} * G * D_{\text {input }}
\end{aligned}
$$


The constraints included in this formulation are required to address the issues related to loop interactions and dead-time. The first constraint, Equation 30, is related to the loop interactions. Here, $\otimes$ denotes the element-wise matrix multiplication and $a c$ is the number of active constraints. $\alpha$ and $\beta$ are real vectors. The gain matrix is arranged such that the pairings of the manipulated variables with the active constraints lie upon the diagonal elements. As the assumption made in this work is that active constraints control must be the highest priority, i.e., the best possible control performance, this constraint is only applied to the active constraint control loops. This constraint serves two important roles in the primary variable selection problem. As the measure chosen for controllability is based upon a process gain matrix that is calculated with active constraint controls in place, the effect of the active constraint control on the remaining controlled variables is accounted for in the gain matrix. However, the effect of the additional controlled variables on the active constraint control should be accounted for during the controlled variable selection process. RGA analysis can be performed by using the process gain matrix without the active constraint control, thereby allowing one to determine how the selected primary controlled variables will affect the performance of the active constraint controls. With this constraint, one can specify the maximum extent of degradation in the active constraint control that is acceptable.

$$
\alpha_{i} \leq\left(G \otimes G^{-\mathrm{T}}\right)_{i, i} \leq \beta_{i} \text { for } i=1: a c
$$

A second constraint, Equation 31, is used to address the associated dead-time of the paired manipulated variable with the controlled variable. Here, $h\left(u_{i}, y_{j}\right)$ is a function of the $i^{\text {th }}$ input and $j^{\text {th }}$ output that describes or estimates the dead-time between $u_{i}$ and $y_{j} . \chi_{i j}$ is a user specified constraint on the loop dead-time of $u_{i}$ and $y_{j}$. This constraint is required to ensure good controllability of the resulting process. This constraint can be formulated in the form available in the existing literature (Yelchuru \& Skogestad, 2012). Work is ongoing in our group in the development of a rigorous, generically applicable means of addressing this issue for controlled variable selection.

$$
h\left(u_{i}, y_{j}\right) \leq \chi_{i j}
$$

If the process model is nonlinear, the linear model of the process and disturbance gain matrices required for the evaluation of the economic and controllability selection criteria can be generated by linearizing around the nominal conditions. The Hessians of the cost function may be 
calculated analytically or numerically. For numerical calculation of the Hessians, the gain matrix of the terms in the cost function can be calculated at the nominal operating point. The gain matrix can then be recalculated after a small change in either an input variable or disturbance variable is introduced. This should be undertaken for all input variables and disturbance variables. From the resulting set of gain matrices, the Hessians can then be calculated numerically.

From the above mentioned measures of economic and control performance, the mixed-integer, multi-objective optimization problem shown in Equation 32 is formulated. The method used to solve this constrained, multi-objective optimization problem and the contributions to the existing methods will be discussed in the next section. The solution of this optimization problem will be a set of Pareto-optimal solutions. It should be mentioned that further analysis of these sets is required by using the nonlinear process model to make a final selection of the primary controlled variables.

$$
\min _{\mathrm{P}_{n}}\left\{L_{\text {average }}\left(P_{n}\right), J_{c}\left(P_{n}\right)\right\}
$$

subject to:

$$
\begin{aligned}
& L_{\text {average }}=\frac{1}{6\left(n_{y}+n_{d}\right)} \|\left[\left(G_{p} J_{u u}^{-\frac{1}{2}}\right)^{-1}\left[\left(G_{p} J_{u u}^{-1} J_{u d}-G_{d}\right) W_{d} W_{e}\right] \|_{F}^{2}\right. \\
& J_{c}\left(P_{n}\right)=\underline{\sigma}^{-1}\left(\hat{G}\left(P_{n}\right)\right) \\
& \hat{G}=D_{\text {output }} * G_{A C} * D_{\text {input }} \\
& \alpha_{i} \leq\left(G\left(P_{n}\right) \otimes G\left(P_{n}\right)^{-\mathrm{T}}\right)_{i, i} \leq \beta_{i} \text { for } i=1: a c \\
& h\left(u_{i}, y_{j}\right) \leq \chi_{j}
\end{aligned}
$$

\subsubsection{A Posteriori Analysis}

From the multi-objective optimization, multiple controlled variable sets will be identified. However, a linear process model is used in this approach. In order to finalize the selection, the economic and control performance of the controlled variable sets are evaluated by using the 
nonlinear process model during the a posteriori analysis. At the end of this analysis, several feasible sets may be found that show acceptable economic and control performance. At this point, process knowledge and insight may be used to make the final selection of the primary controlled variable set.

During the a posteriori analysis, first the economic loss is determined through the evaluation of the cost function at varying magnitudes of the identified disturbances with the primary controlled variables being held constant by using the manipulated variables (Araujo, Govatsmark, \& Skogestad, 2007). This determines the actual loss that would be expected from a constant setpoint policy for each of the controlled variable sets.

In addition to nonlinear evaluation of economic performance, the control performance must also be determined. Evaluation of controllability by using the nonlinear process model is accomplished via the linearization of the process at multiple optimal operational points and at suboptimal operational points where the controlled variables are held constant by the manipulated variables. These two methods account for the two possible situations the process may encounter: the first when there is a an RTO updating the setpoints of the supervisory control layer and the second when there is no RTO or updated setpoints are not yet available from the RTO. It should be noted that the second situation is the primary motivation for selecting the selfoptimizing controlled variables. Two linear models should be generated for all the mentioned cases under the two possible situations mentioned before, one with the active constraints controlled and another without the active constraints controlled. As with the two models used in the multi-objective optimization, both models are required to get a complete understanding of how the process will behave. The process gain matrix that is calculated with active constraint controls in place addresses the effect of the active constraint control on the remaining controlled variables accounted for in the gain matrix. However, the effect of the remaining controlled variables on the active constraint control is not. With these models, the control performance of 
each controlled variable set can be examined at multiple operational points as well as under the condition of an RTO and without an RTO.

To begin, the gain matrices with the active constraint controlled are scaled in the same manner as mentioned in Section 3.1.1.1. The minimum singular values are then calculated for all of these cases, i.e., all feasible controlled variable sets under optimal and suboptimal operations. This allows for the determination of how the worst input direction associated with the controlled variable set changes as the disturbances affect the process. This is required as the controlled variable sets could have poor control performance as the disturbances of various magnitudes affect the process.

It is possible that a number of controlled variable sets perform satisfactorily as evaluated by the nonlinear process model. Further analysis can be done for screening the controlled variables by considering other constraints that have not been considered before such as control complexity, ease of measurement and/or maintenance of the selected controller variable set, and process insight to determine which set will be the final primary controlled variable set. As most of these issues are process dependent, it is not possible to address all situations in a methodical manner. However, it is possible to estimate the control complexity of a primary controlled variable set. To estimate the likely control complexity required for adequate control, the control performance is evaluated considering decentralized control. It is determined how $G_{p}$ changes for a controlled variable set as operations change. To determine this, the appropriate input-output pairings needs to be determined. This can be accomplished via an RGA analysis at the nominal operation point. With the pairings determined, corresponding elements of the scaled gain matrix are examined. This examination is similar to examinations done in Equations 25 and 26. However, in those cases, the examination was limited to evaluation whether an input existed that had the power to reject the disturbances on the controlled variable and to drive the controlled variable within a desired range. In this case, the pairings are determined from the RGA analysis and the 
evaluation is in relation only to that particular element. Specifically, if the element is less than 1, the control performance of that loop will likely be poor. This examination is then undertaken for the remaining gain matrices that were computed at the off-design conditions. An RGA analysis is done for these off-design operations to determine how the loop interactions change as the disturbances affect the process. Finally, it is also important to note if the gain of any of the control loops will flip during disturbance operations. It should be noted that the analyses mentioned in this paragraph can be used to reduce the number of controlled variable sets further, but are not necessarily required as even if loop interactions become large, loop gains deteriorate, or loop gains change sign, this does not necessarily imply that the system will have poor control performance. It does, however, imply the need for a more complex control structure for the supervisory control layer. This can become relevant especially if the economic and control performance of several of the candidate sets are nearly equivalent. If, for example, two controlled variable sets show nearly equivalent economic and control performance, one would choose the controlled variable sets that requires the least complex supervisor control layer.

\subsection{Secondary Controlled Variable Selection}

In this work, a new method of secondary controlled variable selection has been developed that considers the servo and regulatory control performance of the closed loop system directly and optimizes the regulatory control performance as measured by the scaled integral absolute error (IAE). Additionally, issues related to loop interactions are addressed by relative gain array (RGA) constraints on the supervisory and regulatory control layers. This method consists of three stages as summarized below.

- A Priori Analysis

○ This stage comprises of formulation and implementation of a subset selection constraint. This subset selection constraint is an integer constraint that is used 
to determine if a manipulated variable can be used for control of a candidate controlled variable. Servo and regulatory control performance criteria, as well as process insight, can be used to formulate this constraint.

- Set Selection

A new objective function is formulated for selection of the secondary controlled variables. This new objective function is sum of scaled integral absolute errors (IAEs) of the primary controlled variable loops with secondary controlled variables used as manipulated variables. The IAEs are scaled according to the economic importance of the associated primary controlled variable. In this way, both the economic performance and control performance of the control system are addressed in a single objective function.

- Loop interactions are included within the framework. An RGA analysis is included within the formulation to ensure minimal loop interactions exist within the regulatory and supervisory control layers.

- An approach by which the subset selection constraint may be implemented within a branch and bound algorithm is discussed. In addition, methodologies by which this constraint may be used for pruning of supernodes (nodes with a large set of possible solutions) within a branch and bound algorithm are also discussed.

- A Posteriori Analysis

$\circ$ Evaluation of the controllability of the system at off-design operating conditions. 
- Evaluation of the dynamic performance of the secondary controlled variables within the nonlinear process model.

\subsubsection{A Priori Analysis}

The a priori analysis is divided into four steps: identification of a candidate set of secondary controlled variables and disturbances, generation of a subset selection constraint, identification of input-output models, and calculating and scaling of the IAE of the cascaded primary control loop.

\subsubsection{Identification of a Candidate Set of Secondary Controlled Variables and Disturbances}

For the identification of the candidate set of secondary controlled variables, process insight is required. It is at this stage that one must decide which controlled variables will be considered for further analysis and this decision is best made with process insight. While it is possible to screen a large number of candidate variables by using the proposed three stage method, inclusion of certain candidate variables may not add any value. At this stage, only those variables that are obviously not candidate secondary controlled variables are excluded from the candidate set. Applying process insight in this manner simplifies and accelerates the entire procedure of secondary controlled variable selection; however, it is not required. Likewise, identification of disturbances to the process also requires process insight. The disturbances here do not necessarily need to be the same as those that were considered during primary controlled variable selection. The disturbances considered during primary controlled variable selection are related to higher level operations and disturbances. At this level, it is of more concern to reject local disturbances and not allow them to propagate through the process, eventually affecting the primary controlled variables and/or making the process unstable. Unlike the generation of a candidate set of secondary controlled variables, which does not necessarily require process 
insight, the proposed procedure for secondary controlled variable selection requires the identification of process disturbances, therefore requiring a degree of process insight.

\subsubsection{Subset Selection Constraint}

Here, a subset selection constraint is defined. Let $Q$ be a set of $n_{c v}$ vectors of length $n_{u}$. Here, $n_{c v}$ is the number of candidate secondary controlled variables and $n_{u}$ is the number of manipulated variables. If the $j^{\text {th }}$ element of the $i^{\text {th }}$ set is a logical 1 , the corresponding pairing of the $i^{\text {th }}$ input to the $j^{\text {th }}$ output is feasible; otherwise, if it is a logical 0 , it is not feasible. Servo and regulatory control performance of all pairings of manipulated variable to secondary controlled variable are used to formulate this constraint. All pairings of a manipulated variable to a controlled variable are checked to determine if Equations 1 and 2 are true. Equations 33 and 34 reflect whether the controlled variable/manipulated variable pairing has acceptable servo and regulatory control

performance, respectively. If both of these Equations are true, the pairing of the $i^{\text {th }}$ input to the $j^{\text {th }}$ output is defined as feasible, i.e., $Q_{i, j}$ is a logical 1 , otherwise $Q_{i, j}$ is a logical 0 . Here, $G_{p 2}$ and $G_{d 2}$ are the process gain and disturbance gain matrices of the secondary controlled variables, respectively. This evaluation is undertaken for all the $n_{u}$ input variables and $n_{c v}$ output variables. It should be noted that additional constraints may be used for the formulation of this subset selection constraint. This can include, but may not be limited to, servo and regulatory control constraints at off-design operations and/or gain switching constraints, i.e., checking whether the gain of a manipulated variable to a secondary controlled variable changes direction at certain operating conditions.

$$
\begin{gathered}
\left(G_{p 2}\right)_{i, j} \geq 1 \\
\left(G_{p 2}\right)_{i, j} \geq \max \left(\left|\left(G_{d 2}\right)_{i}\right|\right)
\end{gathered}
$$

With the subset selection constraint formulated, implementation of the constraint within a branch and bound algorithm follows a similar procedure as discussed in our previous work (Jones et al., 
2013). The difference between this application and the previous work is that this subset selection constraint is not based upon a predefined number of controlled variables that may be selected from a subset. In this work, the constraint defines whether a manipulated variable can or cannot be used for control of a particular controlled variable. Similar to the previous work, this can be used for pruning within the branch and bound algorithm.

Here it will be explained how the proposed subset selection constraint can be used for pruning within the branch and bound algorithm. The first, and easiest, case is when $\sum Q_{k, j}\left(S_{n}\right)=0: \forall k \in\left\{1: n_{c v}\right\}$ is true. Here, $S_{n}$ is the union of $F$ and $C$, where $F$ and $C$ are the fixed and candidate sets, respectively, of the node $n$. If this equation is found to be true for any node, that node is infeasible as are all of its subsets. This is due to a controlled variable within the fixed set, $S_{n}$, not being controllable by any manipulated variable, as defined by the subset selection constraint.

For the second case, if for a particular $n$ node it is true that $\sum Q_{k, j}\left(S_{n}\right)=1: \forall k \in\left\{1: n_{c v}\right\}$ there exists an element within the fixed set that can only be controlled by one manipulated variable. Let $r$ denote this manipulated variable. Due to this, all controlled variables within the candidate set, $C_{n}$, that can only be controlled by the $r^{\text {th }}$ manipulated variable are no longer feasible candidate controlled variables within the particular node. Therefore, the candidate set $C_{n}$ is redefined as $C_{n} \backslash\left(Q_{k, j} \cap \neg\left(\left(\bigcup_{p \subseteq K} Q_{p, j}\right) \backslash Q_{k, j}\right)\right)$. With this pruning complete, the $r^{\text {th }}$ column of $Q_{k}$ is removed from the node and it is again checked if $\sum Q_{k}\left(S_{n}\right)=0: \forall k \in\left\{1: n_{c v}\right\}$ or $\sum Q_{k}\left(S_{n}\right)=1: \forall k \in\left\{1 n_{C v}\right\}$. Depending upon the outcome, the pruning continues until there is no $k$ such that $\sum Q_{k}\left(S_{n}\right)=0$ nor $\sum Q_{k}\left(S_{n}\right)=1$ is true. 


\subsubsection{Input-Output Model Identification}

For the calculation of the IAEs of all pairings of $u-y_{2}-y_{1}$, input-output models need to be identified. The models required are disturbance models, input to secondary controlled variables, and input to primary controlled variables. In addition, models of the sensors are also required to accurately capture the effect of measurement delays on the overall system performance.

\subsubsection{Calculation of IAEs}

With the identified input-output models, the IAE of all possible pairings of $u-y_{2}-y_{1}$ must be calculated. This is required as one set of secondary controlled variables may result in different pairings of $u-y_{l}$ from another set. The IAEs required are for servo and regulatory control. The block flow diagram shown in Figure 5 is used to model the system where $G_{1}$ and $G_{2}$ are the transfer functions of the primary and secondary controlled variables, respectively, $G_{C 1}$ and $G_{C 2}$ are the controllers for the primary and secondary controlled variables, respectively, and $H_{l}$ and $\mathrm{H}_{2}$ are the models of the measurement device for the primary and secondary controlled variables, respectively. It should be noted that this model is based upon the assumption that the disturbances affect the outputs and not the inputs. The block flow diagram should be suitably modified if that is not the case. From this model, it can be shown that the error of the primary controlled variable is defined as Equation 35.

$$
e_{1}(s)=\frac{1+G_{C 2} G_{2} H_{2}+G_{C 1} G_{C 2} G_{1} H_{1}-G_{C 1} G_{C 2} G_{1}}{1+G_{C 2} G_{2} H_{2}+G_{C 1} G_{C 2} G_{1} H_{1}}\left(y_{1}\right)_{r e f}(s)+\frac{G_{d 1}+G_{d 1} G_{C 2} G_{2} H_{2}-G_{d 2} G_{C 2} G_{1} H_{2}}{1+G_{C 2} G_{2} H_{2}+G_{C 1} G_{C 2} G_{1} H_{1}} d(s)
$$

Obviously, the design of the controllers $G_{C l}$ and $G_{C 2}$ will affect the results. From these transfer functions, it is now possible to calculate the IAE of the servo and regulatory performance for all pairings of $u-y_{2}-y_{1}$. For the regulatory performance, each disturbance can be applied one at a time and the resulting IAEs can then be summed. The IAEs for the regulatory control are stored in the three dimensional matrix $I A E_{\text {reg. }}$. The IAEs for the servo control are stored in the three dimensional matrix $I A E_{\text {servo. }}$. The economics of the process are used for the determination of the applied scaling to the IAEs. For primary controlled variables that are active constraints, the 
scaling applied is $\left|\frac{\delta J}{\delta \lambda}\right|$, where $J$ is the cost function of the process that was defined in Jones et al. [2013] for the selection of the primary controlled variables and $\lambda$ is the active constraint. For primary controlled variables that are self-optimizing, the applied scalings are $\sigma\left(J_{u u}^{1 / 2} G^{-1}\right)$, where the $G$ is the process gain matrix with the active constraints controlled and $J_{u u}$ is the Hessian of the cost function with respect to the inputs used to control the self-optimizing controlled variables, again the active constraint controls are in place. Applying this scaling methodology provides insight not only into the control performance of the process, but also how that control performance will impact the economics of the process as well. In this way, if the control performance of one loop deteriorates during set selection in favor of improving the performance of another that more drastically impacts the economics of the system, then that can be accounted for in the set selection algorithm.

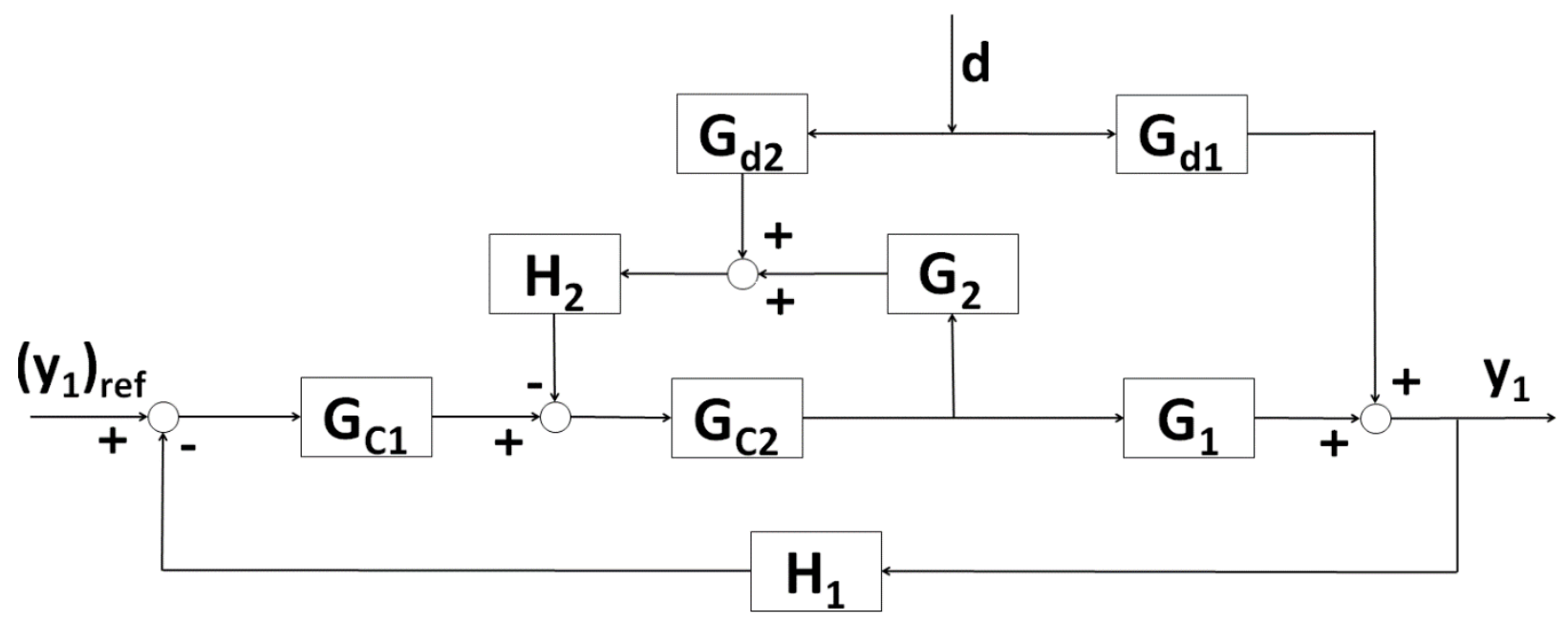

Figure 5. Block flow diagram of cascaded primary control variable loop

\subsubsection{Set Selection}

During set selection, it must be ensured that the servo and regulatory control performance of the regulatory control layer is satisfied, minimal loop interactions exist within the regulatory control layer so as to ensure that decentralized control will provide adequate control performance, 
satisfactory loop interaction exists within the supervisory layer to minimize control problems, and the summation of the scaled ISEs is minimized to ensure optimal control/economic performance. Each of these points will be discussed in detail within this section.

\subsubsection{Servo and Regulatory Control of Regulatory Control Layer}

First, to address the issue related to servo and regulatory control performance, two constraints are imposed on the selection of secondary controlled variables. The first, Equation 36, stipulates that a manipulated variable, within a bound, must be able to drive a secondary controlled

variable within a desired range. The second constraint, Equation 37, stipulates that a manipulated variable, within a bound, must exist that can reject the effects of a disturbance on the secondary controlled variable.

$$
\begin{gathered}
\underline{\sigma}\left(G_{p 2}\right) \geq 1 \\
\bar{\sigma}\left(G_{p 2}^{-1} G_{d 2}\right) \leq 1
\end{gathered}
$$

\subsubsection{Loop Interaction and Control Pairings}

Loop interaction is considered within this selection framework by performing an RGA analysis and by considering the RGA number. The loop interactions at both the regulatory and supervisory control layers should be considered. Therefore, two RGAs are required, one for the regulatory control layer and another for the supervisory control layer. As the selection of secondary controlled variables will affect both RGAs, two constraints must be applied. For the regulatory control layer, the constraint is defined as Equation 38 and for the supervisory control layer, the constraint is defined as Equation 39. Again, because the design of the regulatory control layer will directly affect the design of the supervisory control layer, this effect should be considered while selecting secondary controlled variables.

$$
\alpha_{i} \leq\left(G_{p 2} \otimes G_{p 2}-\mathrm{T}\right)_{i, i} \leq \beta_{i}
$$




$$
\chi_{i} \leq\left(G_{p 1} G_{p 2}^{-1} \otimes\left(G_{p 1} G_{p 2}^{-1}\right)^{-\mathrm{T}}\right)_{i, i} \leq \delta_{i}
$$

In addition to ensuring good control performance of the regulatory controls and supervisory controls, an RGA analysis is also required for the evaluation of the objective function. The evaluation of the objective function is dependent upon the connection of input variable to secondary controlled variable to primary controlled variable. These connections are dependent upon the secondary controlled variables that are selected; since one set of secondary controlled variables may result in a completely different pairing of $u-y_{1}$ as another set. For determining how these variables are connected, the RGA analysis is used. For determining both the optimal pairing of input variable to secondary controlled variable and secondary controlled variable to primary controlled variable, the minimization of the RGA number can be considered to be the objective. However, as these evaluations will have to be done within the branch and bound algorithm and they are relatively high in computation cost, solution times can become quite high. To address this issue, the subset selection constraint can be used in addition to the RGA constraints to help reduce the overall computation costs, instead of calculating the RGA number. Here it will be explained how the RGA constraints and subset selection constraint can be used to more efficiently determine optimal pairings in comparison to enumeration of the RGA number. However, it should be noted that the use of the subset selection constraint can only be used in this way for the regulatory control layer but not for the supervisory control layer. First, a matrix, $P$, is defined based upon the subset selection matrix, $Q$. Here, $P$ is made up of the corresponding secondary controlled variable set associated with a node from $Q$. Next, the RGA for the regulatory and supervisory control layers are calculated and stored.

If any element of the summation of the rows or columns of the matrix $P$ is equal to 1 , then, based upon the subset selection constraint, the only feasible pairing is associated with the location of that particular element. This is due to either a controlled variable being only controllable by a 
single manipulated variable or a manipulated variable only able to control a single controlled variable. As this pairing is required by the subset selection constraint, the corresponding row and column of the matrix $Q$ are then removed. This evaluation continues until there are no exclusive pairings of manipulated variables to secondary controlled variables. Based on the updated $Q$, a new matrix $R$ is calculated. This new matrix is a truth matrix defined as whether an element of the RGA is within the bounds of the constraint defined in Equation 36, if it is the corresponding element of $P$ is a logical 1 otherwise a logical 0 . With this newly defined $R$ matrix, the procedure is applied again. For the same reason as discussed above, this is due to only a particular pairing being feasible without violating the RGA constraint. Unlike the subset constraint, the RGA constraint can also be used to determine the pairings of secondary variables to primary controlled variables.

\subsubsection{Closed Loop System IAEs}

Finally, the IAE of the closed loop system must be determined. This can be done by using the stored IAE values calculated and scaled during the a priori analysis. The stored threedimensional matrix contains the IAE values of all the possible pairings, used in conjunction with the RGA analysis, and can be used to determine the total IAE of the closed loop system. This method of calculating the IAE assumes that there are no interactions between the loops. This is not necessarily required, as it is possible to calculate the closed loop IAE with loop interactions, however, that is far more computationally expensive and, for large scale systems, may become computationally intractable. By making the assumption that there are no loop interactions, it is possible to make the IAE calculations offline and use the three dimensional matrix of IAE values as a lookup table for the evaluation of the objective function. Here the objective is the minimization of the summed IAEs related to the regulatory control performance of the supervisory control layer. The IAEs related to the servo control performance can be used as a constraint within the formulation, if desired. 


\subsubsection{Formulation of the Optimization Problem}

A constrained optimization problem is formulated for the generation of a set of secondary controlled variables. The objective is the minimization of the summed and scaled IAEs of the primary control loops as described in Section 3.2.2.3. The constraints included in this formulation are required to address the issues of loop interactions, both at the regulatory and supervisory control layers, and servo control performance of the primary control loop. In addition, the subset selection constraint, as defined in Section 3.2.1.2, is also included within this formulation. With this, the constrained, mixed integer optimization problem shown as Equation 40 is solved to determine the optimal set of secondary controlled variables. For all solutions investigated during the course of the optimization, shown as Equation 41, the optimal pairings of $u-y_{2}$ and $y_{2}-y_{1}$ must be determined, subject to the subset selection constraint. The constrained, mixed integer optimization problem shown as Equation 41 is used to determine the optimal pairing of $u-y_{2}$ and the mixed integer optimization problem shown as Equation 42 is used to determine the optimal pairing of $y_{1}-y_{2}$.

$$
\begin{aligned}
& \min _{y_{2}} \sum \operatorname{IAE} E_{R E G}\left(y_{2 s}\right) \\
& \text { subject to: } \\
& Q_{i, i}=1 \text { for } i=1: n_{u} \\
& \alpha_{i} \leq\left(G_{p 2} \otimes G_{p 2}^{-\mathrm{T}}\right)_{i, i} \leq \beta_{i} \text { for } i=1: n_{u} \\
& \gamma_{i} \leq\left(G_{p 1} G_{p 2}^{-1}\left(y_{2 s}\right) \otimes\left(G_{p 1} G_{p 2}^{-1}\left(y_{2 s}\right)\right)^{-\mathrm{T}}\right)_{i, i} \leq \delta_{i} \text { for } i=1: n_{u} \\
& \sum \operatorname{IAE} E_{S E R V O}\left(y_{2 s}\right) \leq \varepsilon \\
& \underline{\sigma}\left(G_{p 2}\left(y_{2}\right)\right) \geq 1 \\
& \bar{\sigma}\left(G_{p 2}^{-1}\left(y_{2}\right) G_{d 2}\right) \leq 1
\end{aligned}
$$




$$
\begin{gathered}
\min _{k}\left\|\left(G_{p 2}\left(y_{2}\right) \otimes G_{p 2}^{-\mathrm{T}}\left(y_{2}\right)\right)_{k}-I\right\|_{S U M} \\
\text { subject to: } \\
Q_{i, i}=1 \text { for } i=1: n_{u} \\
\min _{j}\left\|\left(G_{p 1} G_{p 2}^{-1}\left(y_{2}\right) \otimes\left(G_{p 1} G_{p 2}^{-1}\left(y_{2}\right)\right)^{-\mathrm{T}}\right)_{j}-I\right\|_{S U M}
\end{gathered}
$$

\subsubsection{Posteriori Analysis}

After set selection, multiple sets of secondary controlled variables should be identified. To finalize the selection of a secondary controlled variable set, a posteriori analysis is required since a linear process model under nominal operation is used in the proposed approach. Therefore, the controllability of the sets obtained at the end of the second stage should be evaluated under offdesign operations. From the analysis undertaken during the primary controlled variable selection, the user should have identified the off-design operations, as shown in Jones et al. [2013]. The analysis of controllability consists of the evaluation of the servo and regulatory control performance of the regulatory control layer, an RGA analysis for the regulatory and supervisory control layers, and ensuring that no gain switching occurs between the pairings of $u$ $-y_{2}-y_{1}$. Some, or all, of these analyses may be implemented within the second stage where set selection is performed; however, specification of desired performance under off-design operation can be difficult and the problem can become overly constrained in case of very aggressive constraints. By applying these analyses posteriori, the user can intervene to make the final selection of the controlled variable set.

\subsection{Control Structure Design}

As discussed in Section 2.2.3.1, Gramian based interaction measures can be used to inform the design of the structure of the supervisory control layer. However, within the open literature, no work can be found that proposes a systematic means of using these measures for the design of 
the control structure. In this section, a systematic means of determining the optimal control structure is proposed.

\subsubsection{Gramian Interactions}

An important characteristic of the Gramian based interaction measures is that they are scaling dependent. Therefore, before these measures can be used in a systematic means for the design of the control structure, a systematic means of scaling must be defined. To this end, it is proposed that the Gramian interaction measures should be scaled in such a way that the sum of all elements of any row is the same as the sum of all elements of any column. Scaling in this way ensures that all output variables are considered of equal importance, i.e., one output variable is not considered more important than any other output variables. Additionally, it is assumed that the relative 'power' of all input variables are the same, specifically, all input variables have the same relative gain. This scaling method makes the properties of the Gramian matrices similar to that of the commonly used RGA.

Applying this scaling method to the Gramian interaction matrices now allows for a systematic means of determining the expected control performance of any number of possible control structures.

\subsubsection{Control Structure Complexity}

The Gramian interaction measures provide quantitative insight into the possible control benefits of moving from a simple, decentralized control structure, to a more complex control structures, such as MPC. However, to determine a truly optimal control structure, a quantitative measure of controller complexity is required. This is due to the need to balance control performance with control complexity. A few authors have proposed some measures of controller complexity. The measure proposed by (Skogestad, 2004) is based, largely, on the number of input variables, output variables, and tuning factors of the proposed control structure. However, this measure does not address the issue of computation time required to determine control actions. For this 
reason, the measure of computational expense proposed in this work is based upon the computational time required for the calculation of control actions. For simple PID control, it is assumed that the computation is completed instantaneously. For centralized, MPC based control, it is assumed the computational time required is defined as Equation 43. This measure is based upon the time complexity of the evaluation of an $n$ dimensional optimization problem (Karmarkar, 1984). Using this measure, it is possible to determine a set of Pareto optimal control structures which balance control performance with control complexity.

$$
O\left(n^{2} \ln (n)\right)
$$




\section{Application}

In this section, the application of the above described control system design procedure is applied to an acid gas removal (AGR) unit and the gasification section of the IGCC power plant with $\mathrm{CO}_{2}$ capture.

The IGCC power plant with $\mathrm{CO}_{2}$ capture that is considered in this work is based upon the work of Bhattacharyya et al, 2011. A simplified block flow diagram of the process is shown in Figure 6. The process begins when coal is gasified in the gasifier to generate a raw synthesis-gas stream. This syngas consists of carbon monoxide, carbon dioxide, hydrogen, water, hydrogen sulfide, and other impurities. This raw syngas is then sent to a series of water-gas shift reactors. These reactors promote the water-gas shift reaction, shown as Equation 44. The shifted syngas is then sent to the acid gas removal unit (AGR) where carbon dioxide, ammonia, and hydrogen sulfide are selectively removed from the syngas. The carbon dioxide is sent to the $\mathrm{CO}_{2}$ compressors where it is pressurized and sent for sequestration. The ammonia and hydrogen sulfide are sent to the Claus unit where ammonia is destroyed and hydrogen sulfide is converted to elemental sulfur. The cleaned syngas is then sent to the gas turbine (GT) for power production. The hot tail gas from the GT is then sent to a heat recovery steam generation unit (HRSG) where it is used to raise three pressures of steam for additional power production.

$$
\mathrm{H}_{2} \mathrm{O}+\mathrm{CO} \Leftrightarrow \mathrm{CO}_{2}+\mathrm{H}_{2}
$$




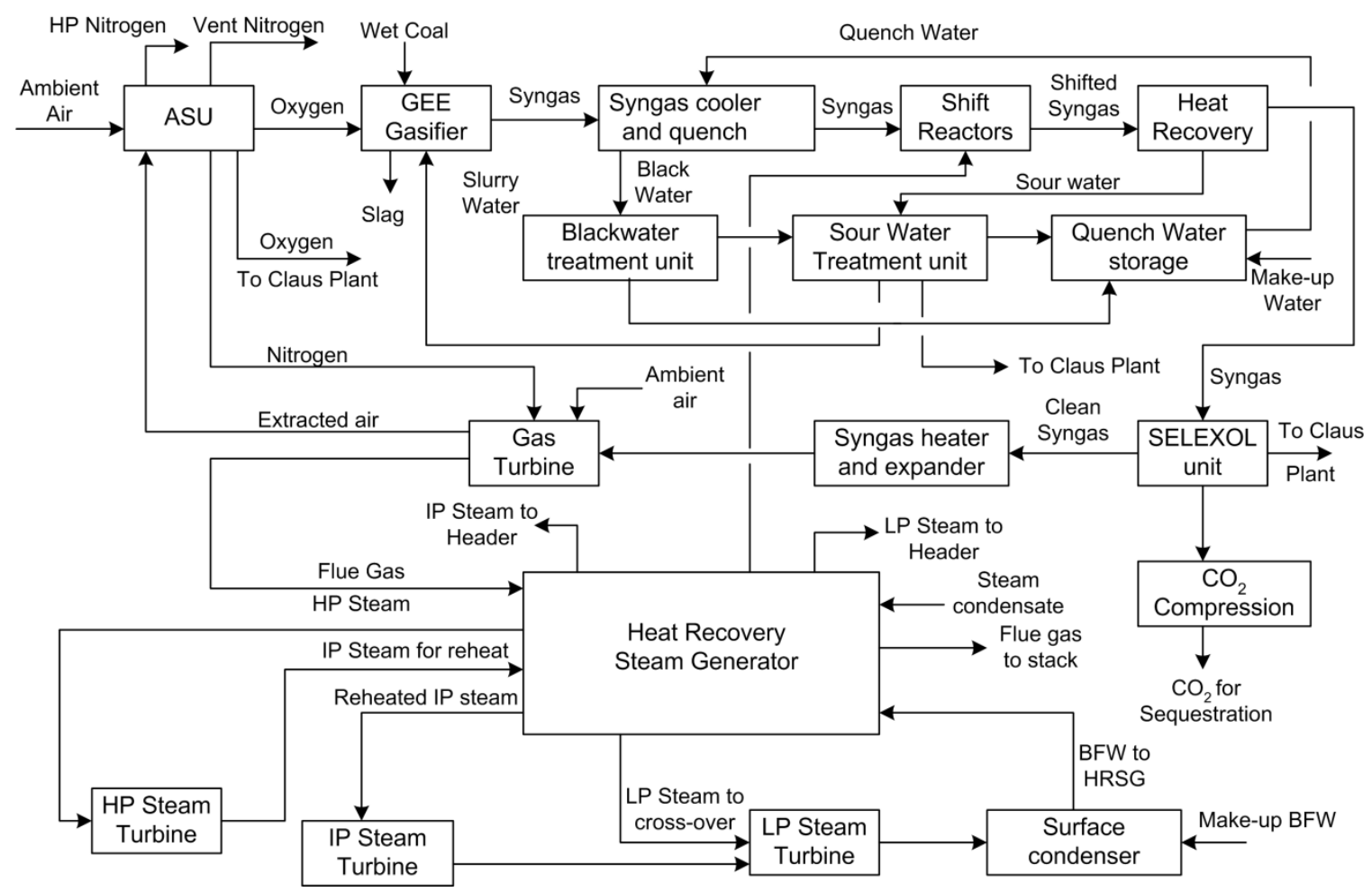

Figure 6: Block Flow Diagram of IGCC with Carbon Capture (Bhattacharyya et al, 2011)

\subsection{Acid Gas Removal (AGR) Unit}

The AGR considered in this work is a dual-stage SELEXOL unit based upon the work of

(Bhattacharyya et al, 2011). A dual-stage Selexol unit, as shown in Figure 7, is used to remove hydrogen sulfide (first stage) and carbon dioxide (second stage) contained in the shifted syngas.

Part of the loaded solvent from the $\mathrm{CO}_{2}$ absorber is sent to a series of four flash vessels. The first flash is for the recovery of most of the hydrogen dissolved in the solvent. The remaining three vessels are used to flash off the dissolved carbon dioxide which is subsequently compressed and sent for sequestration. This semi-lean solvent is then sent back to the $\mathrm{CO}_{2}$ absorber. The remainder of the loaded-solvent from the bottom of the $\mathrm{CO}_{2}$ absorber is sent to the $\mathrm{H}_{2} \mathrm{~S}$ absorber. From the $\mathrm{H}_{2} \mathrm{~S}$ absorber, the solvent is sent to the $\mathrm{H}_{2} \mathrm{~S}$ concentrator, where by means of a pressure swing and using nitrogen as a stripping gas, the majority of the dissolved carbon dioxide is released from the solvent while leaving the majority of the hydrogen sulfide. The stripped gas is recycled back to the $\mathrm{H}_{2} \mathrm{~S}$ absorber. From the concentrator, the solvent is sent to the Selexol stripper, where hydrogen sulfide is stripped from the solvent and sent to a Claus unit to recover elemental sulfur. This thermally regenerated solvent is then pumped, chilled, and sent back to the $\mathrm{CO}_{2}$ absorber. 


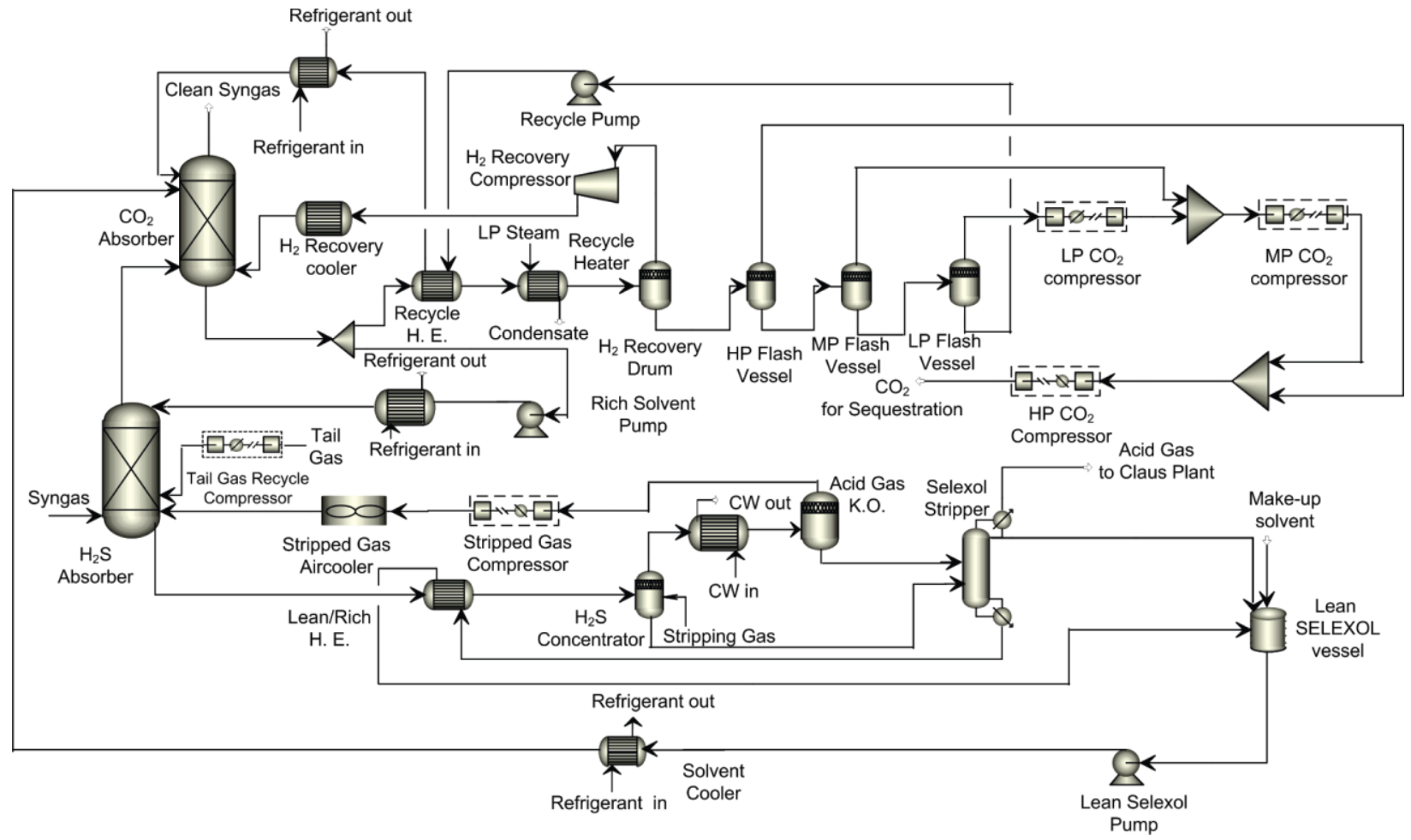

Figure 7: Configuration of Selexol Unit with $\mathrm{CO}_{2}$ Compressors (Bhattacharyya et al, 2011)

The plant model is developed using the PC-SAFT EOS thermodynamics package available in Aspen Engineering Suite (AES). As reported in the AspenTech knowledge base, the parameters used in the EOS are determined by regressing with the experimental data available in the open literature.

\subsubsection{Top-Down Analysis}

\subsubsection{A Priori Analysis}

\subsubsection{Optimization}

There exist two obvious operational objectives that could be considered for the AGR unit. These are the minimization of operational cost or minimization of auxiliary power consumption in the unit. For all the studies considered here, the operational objective considered was the minimization of the operational cost. For this operational objective, associated costs of utilities, feeds, and products are considered. The utilities used within the AGR unit are electricity, steam, cooling water, and refrigerant (ammonia). The steam utility is converted into an equivalent electrical power production for the determination of its cost. This was done by using a steam 
turbine to determine the electrical power generation possible from the steam used for heating duties. The cost for cooling water was also converted into an equivalent electrical cost. This was done by determining the electrical power required to pump the equivalent amount of water required for the heating duties. Ammonia refrigeration duty was likewise converted to an equivalent power requirement by determining the compressor power required to generate the equivalent heating duty. With all utilities converted into an equivalent electrical power requirement, these values were then converted into a cost per hour. The assumed cost of electricity used in these studies is $\$ 0.0943 / \mathrm{kWh}$ (http://www.eia.gov/electricity/monthly). There are three feeds to the AGR unit, the shifted syngas, makeup solvent, and tailgas from the tailgas treatment unit (Bhattacharyya, Turton, \& Zitney, 2011). No cost, or value, was taken for the shifted syngas and tailgas streams. The cost of the makeup solvent was found from (Bucklin and Schendel, 1984). This cost was adjusted based upon the inflation rate to arrive at an equivalent cost in 2011 . The assumed cost of the solvent is $\$ 6.28 / \mathrm{kg}$. There are three product streams from the AGR unit, the cleaned syngas, the Claus unit feed stream, and the $\mathrm{CO}_{2}$ stream. From these product streams, four costs were assumed to be incurred: carbon monoxide sent with the $\mathrm{CO}_{2}$ stream, carbon monoxide sent with the Claus feed, hydrogen sent with the $\mathrm{CO}_{2}$ stream, and hydrogen sent with the Claus feed. These are considered to be losses from the system because these species can be sent to the gas turbine (GT), and subsequently to the heat recovery steam generator (HRSG), for power generation (Bhattacharyya, Turton, \& Zitney, 2011). To determine this power loss, the electrical power output of the GT and steam turbines per unit of lower heating value (LHV) of the clean syngas was determined. This is a measure of the efficiency of the GT and steam turbines as a function of the LHV. This efficiency was used to determine the power output lost from hydrogen and carbon monoxide not being sent with the cleaned syngas based upon the LHV of hydrogen and carbon monoxide. From this analysis, a scalar cost function in terms of dollars per hour was derived and is shown as Equation 45. The units of mass flowrate, power, and heat duty are $\mathrm{kg} \mathrm{h}^{-1}, \mathrm{MW}$, and $\mathrm{MW}$, respectively. Operational constraints of the process are listed within Table 3. Maximum solvent temperature and water content values are taken from (Bucklin and Schendel, 1984). Table 4 shows the disturbances and the magnitudes of the disturbances that are considered likely to affect this AGR unit. The table is prepared considering the expected operational changes in the IGCC power plant. Changes in syngas composition are calculated from assumed variations in inlet temperature of the upstream water-gas shift reactors (Bhattacharyya, Turton, \& Zitney, 2011). 


$$
\begin{aligned}
& -J\left[\frac{\$}{\mathrm{hr}}\right]=6.28\left(\dot{m}_{\text {makeupsolvent }}\right)+0.00982\left(\dot{m}_{\text {steam }}\right) \\
& +17.9 \sum_{k}^{N_{\text {heaters }}} Q_{k}+13.7 \sum_{l}^{N_{\text {refrig }}} Q_{l}+0.836 \sum_{m}^{N_{\text {water }}} Q_{m} \\
& +94.3\left(\sum_{i}^{N_{\text {compressors }}} W_{i}+\sum_{j}^{N \text { pumps }} W_{j}\right) \\
& +3.03\left(\left(\dot{m}_{\mathrm{H}_{2}}\right)_{\mathrm{CO}_{2}}+\left(\dot{m}_{\mathrm{H}_{2}}\right)_{\mathrm{H}_{2} \mathrm{~S}}\right) \\
& +0.374\left(\left(\dot{m}_{\mathrm{CO}}\right)_{\mathrm{CO}_{2}}+\left(\dot{m}_{\mathrm{CO}}\right)_{\mathrm{H}_{2} \mathrm{~S}}\right)
\end{aligned}
$$

Table 3. Operational Constraints

\begin{tabular}{|l|l|}
\hline Constraint & Value \\
\hline Percentage $\mathrm{CO}_{2}$ Capture & $95 \%$ of Inlet $\mathrm{CO}_{2}$ \\
\hline Percentage $\mathrm{H}_{2} \mathrm{~S}$ Capture & $99.95 \%$ of Inlet $\mathrm{H}_{2} \mathrm{~S}$ \\
\hline $\begin{array}{l}\text { Solvent Temperature at the Outlet of } \\
\text { the Refrigeration Coolers }\end{array}$ & $4^{\circ} \mathrm{C}$ \\
\hline $\begin{array}{l}\text { Solvent Temperature at the Outlet of } \\
\text { the Water Coolers }\end{array}$ & $21^{\circ} \mathrm{C}$ \\
\hline $\begin{array}{l}\text { Solvent Temperature at the Outlet of } \\
\text { the Steam Heaters }\end{array}$ & $177^{\circ} \mathrm{C}$ \\
\hline Maximum Compressor Power & $+20 \%$ of nominal \\
\hline Maximum Heat Exchanger Duty & $+50 \%$ of nominal \\
\hline $\begin{array}{l}\text { Maximum Allowable Solvent } \\
\text { Temperature }\end{array}$ & $175^{\circ} \mathrm{C}$ \\
\hline $\begin{array}{l}\text { Maximum Allowable Water Content } \\
\text { of Solvent }\end{array}$ & $6 \mathrm{wt} \%$ \\
\hline Minimum Stripper Pressure & $276 \mathrm{kPa}$ \\
\hline Minimum Claus Feed Purity & \\
\hline
\end{tabular}


Table 4. Disturbances Considered

\begin{tabular}{|c|c|c|c|}
\hline Disturbance & Nominal & Low & High \\
\hline $\mathrm{CO}_{2}$ Capture & $95 \%$ & $91 \%$ & $97 \%$ \\
\hline Syngas Flow Rate & $100 \%$ & $65 \%$ & $105 \%$ \\
\hline $\begin{array}{l}\text { Syngas Composition } \\
\text { (mol fraction) }\end{array}$ & 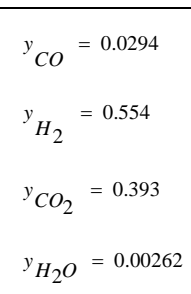 & $\begin{array}{l}{ }^{y}{ }_{C O}=0.0235 \\
y_{H_{2}}=0.556 \\
{ }^{y_{C O}}=0.396 \\
y_{H_{2} O}=0.00263\end{array}$ & $\begin{array}{l}y_{C O}=0.0334 \\
y_{H_{2}}=0.552 \\
y^{{ }^{C} O_{2}}=0.390 \\
y_{H_{2} \mathrm{O}}=0.00263\end{array}$ \\
\hline $\begin{array}{l}\text { Claus Feed Purity } \\
\text { Requirement (mol\% } \\
\mathrm{H}_{2} \mathrm{~S} \text { ) }\end{array}$ & $40 \%$ & $25 \%$ & $60 \%$ \\
\hline Tailgas Flow Rate & $100 \%$ & $75 \%$ & $105 \%$ \\
\hline $\begin{array}{l}\text { Sulfur Content of } \\
\text { Syngas }\end{array}$ & $100 \%$ & $80 \%$ & $110 \%$ \\
\hline
\end{tabular}

Using the 16 DOF available in the AGR unit, the process is optimized at nominal operation and at varying magnitudes of the disturbances as listed in Table 3. Due to the high degree of process nonlinearity and multiple degrees of freedom, this optimization problem is difficult to solve using the default sequential-modular (SM) solution method in Aspen Plus ${ }^{\circledR}$. Instead, the equation-oriented (EO) solver available in Aspen Plus ${ }^{\circledR}$ is used to solve this problem successfully.

With the optimization studies complete, a $27 \%$ reduction in hourly operational cost, at nominal conditions, is accomplished and 10 active constraints are identified. These active constraints are listed in Table 5. 
Table 5. Active Constraints and Pairings

\begin{tabular}{|l|l|}
\hline Active Constraint & Manipulated Variable \\
\hline $\mathrm{CO}_{2}$ Capture & Low Pressure Flash Pressure \\
\hline $\mathrm{H}_{2} \mathrm{~S}$ Capture & Stripper Reboiler Duty \\
\hline Water Content of Solvent & Steam Flow rate \\
\hline $\mathrm{H}_{2} \mathrm{~S}$ Purity to Claus Unit & $\mathrm{H}_{2} \mathrm{~S}$ Concentrator Pressure \\
\hline Stripper Pressure & Stripper Vapor Flow rate \\
\hline Stripper Top Temperature & Stripper Condenser Duty \\
\hline Semi-lean Solvent Cooler Outlet Temperature & Semi-lean Solvent Cooler Duty \\
\hline Loaded Solvent Heater Outlet Temperature & Loaded Solvent Heater Duty \\
\hline Lean Solvent Cooler Outlet Temperature & Lean Solvent Cooler Duty \\
\hline $\mathrm{H}_{2}$ Cooler Outlet Temperature & $\mathrm{H}_{2}$ Cooler Duty \\
\hline
\end{tabular}

\subsection{Control of Active Constraints}

With the active constraints identified, appropriate manipulated variables need to be identified for their control. From the 16 manipulated variables, there are a total of $\frac{16 !}{(16-10) !} \approx 2.906 \times 10^{10}$ possible pairings. However, many of the pairings are obvious, such as cooler heat duties with outlet cooler temperatures. If these obvious pairings are made, there are only $\frac{10 !}{(10-4) !}=5,040$ remaining pairings. To determine the optimal pairings, enumeration of the RGA number in Equation 23 was undertaken for all possible combinations using MATLAB ${ }^{\circledR}$. Since the number of possibilities here are small and the evaluation of the RGA number is computationally inexpensive, enumeration is feasible for all possible combinations using a linearized process model. The linearized model is generated from the nonlinear process model developed within Aspen Plus Dynamics ${ }^{\circledR}$ using a control design interface (CDI) script. The CDI script generates a state-space model and a steady state gain matrix at the current operational point of the nonlinear model. The operational point chosen to carry out this evaluation is the optimal nominal operation identified during the optimization stage of this study. The resulting pairings shown in Table 5 are found to provide the globally minimal RGA number for all the possibilities.

\subsection{Identification of the Candidate Sets of Controlled Variables}

Since the 10 active constraints in Table 5 are added to the controlled variable set, they are removed from the candidate set, reducing the size of the initial set from 282 to 272 . Considering 
the remaining $6 \mathrm{DOF}$, the additional primary controlled variables need to be selected from $\left(\begin{array}{c}272 \\ 6\end{array}\right) \approx 5.3207 \times 10^{11}$ candidate sets. This is a large reduction in the candidate sets from the earlier value of $4.9555 \times 10^{25}$, but direct evaluation in the nonlinear process model is still intractable. The list of the initial controlled variable set is shown in Table 6. The first step taken is the evaluation of Equations 25 and 26 to determine the controllability of each of the individual controlled variables. This analysis eliminates 109 controlled variables from the original list. This reduces the size of the initial list from 272 to 163, reducing the number of possible controlled variable sets from $\left(\begin{array}{c}272 \\ 6\end{array}\right) \approx 5.3207 \times 10^{11}$ to $\left(\begin{array}{c}163 \\ 6\end{array}\right) \approx 2.3734 \times 10^{10}$, a $95 \%$ reduction. 
Table 6. List of Initial Controlled Variable Set

\begin{tabular}{|c|}
\hline Temperature of stages in $\mathrm{CO}_{2}$ Absorber \\
\hline $\mathrm{H}_{2} \mathrm{~S}$ vapor fraction of stages in $\mathrm{CO}_{2}$ Absorber \\
\hline $\mathrm{CO}_{2}$ vapor fraction of stages in $\mathrm{CO}_{2}$ Absorber \\
\hline $\mathrm{H}_{2} \mathrm{~S}$ liquid fraction of stages in $\mathrm{CO}_{2}$ Absorber \\
\hline $\mathrm{CO}_{2}$ liquid fraction of stages in $\mathrm{CO}_{2}$ Absorber \\
\hline Temperature of stages in $\mathrm{H}_{2} \mathrm{~S}$ Absorber \\
\hline $\mathrm{H}_{2} \mathrm{~S}$ vapor fraction of stages in $\mathrm{H}_{2} \mathrm{~S}$ Absorber \\
\hline $\mathrm{CO}_{2}$ vapor fraction of stages in $\mathrm{H}_{2} \mathrm{~S}$ Absorber \\
\hline $\mathrm{H}_{2} \mathrm{~S}$ liquid fraction of stages in $\mathrm{H}_{2} \mathrm{~S}$ Absorber \\
\hline $\mathrm{CO}_{2}$ liquid fraction of stages in $\mathrm{H}_{2} \mathrm{~S}$ Absorber \\
\hline Pressure of $\mathrm{H}_{2}$ Recovery Flash \\
\hline Temperature of $\mathrm{H}_{2}$ Recovery Flash \\
\hline $\mathrm{H}_{2}$ vapor fraction of $\mathrm{H}_{2}$ Recovery Flash \\
\hline $\mathrm{CO}_{2}$ vapor fraction of $\mathrm{H}_{2}$ Recovery Flash \\
\hline $\mathrm{H}_{2}$ liquid fraction of $\mathrm{H}_{2}$ Recovery Flash \\
\hline $\mathrm{CO}_{2}$ liquid fraction of $\mathrm{H}_{2}$ Recovery Flash \\
\hline Pressure of High Pressure Flash \\
\hline Temperature of High Pressure Flash \\
\hline $\mathrm{H}_{2}$ vapor fraction of High Pressure Flash \\
\hline $\mathrm{CO}_{2}$ vapor fraction of High Pressure Flash \\
\hline $\mathrm{H}_{2}$ liquid fraction of High Pressure Flash \\
\hline $\mathrm{CO}_{2}$ liquid fraction of High Pressure Flash \\
\hline Pressure of Medium Pressure Flash \\
\hline Temperature of Medium Pressure Flash \\
\hline $\mathrm{H}_{2}$ vapor fraction of Medium Pressure Flash \\
\hline $\mathrm{CO}_{2}$ vapor fraction of Medium Pressure Flash \\
\hline $\mathrm{H}_{2}$ liquid fraction of Medium Pressure Flash \\
\hline $\mathrm{CO}_{2}$ liquid fraction of Medium Pressure Flash \\
\hline Pressure of Low Pressure Flash \\
\hline Temperature of Low Pressure Flash \\
\hline
\end{tabular}




\begin{tabular}{|l|}
\hline $\mathrm{H}_{2}$ vapor fraction of Low Pressure Flash \\
\hline $\mathrm{CO}_{2}$ vapor fraction of Low Pressure Flash \\
\hline $\mathrm{H}_{2}$ liquid fraction of Low Pressure Flash \\
\hline $\mathrm{CO}_{2}$ liquid fraction of Low Pressure Flash \\
\hline Temperature of stages in $\mathrm{H}_{2} \mathrm{~S}$ Concentrator \\
\hline $\mathrm{H}_{2} \mathrm{~S}$ vapor fraction of stages in $\mathrm{H}_{2} \mathrm{~S}$ Concentrator \\
\hline $\mathrm{CO}_{2}$ vapor fraction of stages in $\mathrm{H}_{2} \mathrm{~S}$ Concentrator \\
\hline $\mathrm{H}_{2} \mathrm{~S}$ liquid fraction of stages in $\mathrm{H}_{2} \mathrm{~S}$ Concentrator \\
\hline $\mathrm{CO}_{2}$ liquid fraction of stages in $\mathrm{H}_{2} \mathrm{~S}$ Concentrator \\
\hline $\mathrm{Temperature}$ of stages in Selexol Stripper \\
\hline $\mathrm{H}_{2} \mathrm{~S}$ vapor fraction of stages in Selexol Stripper \\
\hline $\mathrm{CO}_{2}$ vapor fraction of stages in Selexol Stripper \\
\hline $\mathrm{H}_{2} \mathrm{~S}$ liquid fraction of stages in Selexol Stripper \\
\hline $\mathrm{CO}_{2}$ liquid fraction of stages in Selexol Stripper \\
\hline $\mathrm{Split}_{\text {Fraction of Solvent at Bottom of } \mathrm{CO}_{2} \text { Absorber }}$ \\
\hline Ratio of Syngas - Solvent inlets to $\mathrm{CO}_{2}$ Absorber \\
\hline
\end{tabular}

\subsubsection{Selection of the Pareto-Optimal Controlled Variable Sets}

At this stage, the optimization problem defined in Equation 32 is solved. In the formulation of the optimization problem, the constraints shown within Equations 30 and 31 must be defined. An acceptable maximum degree of loop interactions in the active constraint controls was sought by using $\alpha_{i}=0.5, \beta_{i}=2: \forall i$ for the constraint defined by Equation 30 . Due to this, the evaluation of this constraint is only carried out at terminal nodes to ensure the found solution does not violate the constraint.

In the formulation of the constraint defined by Equation 31, process insight is required. The constraint accounts for the dead-time associated with the effect of a manipulated variable on controlled variables. The constraint that has been formulated for this application is shown as Equation 46. This constraint, as currently formulated, requires a degree of process insight to be applied. For this application, this constraint is formulated so that only a specified number of controlled variables may be selected from a subset of the candidate set. $Q$ is a set of $K$ logical vectors of size $1 \times n_{c v}$ where $n_{c v}$ is the number of candidate controlled variables. These logical 
vectors define whether a controlled variable is a member of the subset. $\chi$ is a set of natural numbers that defines how many controlled variables may be selected from the $K$ subsets. This constraint is similar to what has been proposed by (Yelchuru \& Skogestad, 2012) as a restriction on the number of measurements used from different process sections. However, in that case, the constraint is a formulated as an equality constraint, for this case, a more generically applicable version of this constraint is proposed. This constraint does not necessarily require the selection of a fixed number of controlled variables from a subset. A benefit of this constraint, in addition to ensuring good control performance by addressing issues of large dead-times, is the reduction in number of feasible solutions within the search space.

$$
\chi_{k} \geq \sum_{Q_{k}} P_{n}: \forall k \in K
$$

A means of using the constraint, shown as Equation 46, for the pruning of nodes within the branch and bound algorithm will now be formulated. For all nodes that have been upwardly branched it is checked to determine whether Equation 47 is true. If Equation 47 is true, $C_{n}$ is redefined as $C_{n} \backslash\left(Q_{k} \cap \neg\left(\left(\bigcup_{p \subseteq K} Q_{p}\right) \backslash Q_{k}\right)\right)$. This eliminates all controlled variables that are exclusive to the $k^{\text {th }}$ subset. This can be done because if a controlled variable is exclusively a member of a subset that already has the maximum number of its members in the fixed set, it is not possible to obtain a solution within that node that includes an exclusive variable. That is due to the fact that it will violate Equation 46. The inclusion of this pruning method does not change the monotonicity of any objective function nor constraints that may be used for pruning. This is because when $j \supset i$ and $\left(\sum F_{n}+\sum\left(C_{n} \backslash i\right)\right) \geq\left(\sum F_{n}+\sum\left(C_{n} \backslash j\right)\right)>n_{u}$, where $n_{u}$ is the number of inputs, it must be that $\underline{T}\left(\left(F_{n} \cup C_{n}\right) \backslash j\right) \geq \underline{T}\left(\left(F_{n} \cup C_{n}\right) \backslash i\right) \geq \underline{T}\left(F_{n} \cup C_{n}\right)$ if the function $\underline{T}$ is monotonic. As this pruning method is only applied to the node that has been upwardly branched, it has no effect on the node branched downwardly. If it is found that after applying this pruning method that $\sum F_{n}+\sum C_{n} \leq n_{u}$ the node is pruned as it contains no feasible solutions.

$$
\sum Q_{k}\left(F_{n}\right)=\chi_{k}: \forall k \in K
$$

A degree of freedom analysis is undertaken for the process units within the AGR unit so as to implement the constraint defined within Equation 46. For the $\mathrm{CO}_{2}$ absorber section, there are 3 
degrees of freedom available: the $\mathrm{H}_{2}$ recovery flash pressure, the semi-lean solvent flow, and the lean solvent flow. For the $\mathrm{H}_{2} \mathrm{~S}$ absorber, one degree of freedom is available: the lean solvent flow. For the $\mathrm{H}_{2} \mathrm{~S}$ concentrator, there is one degree of freedom available: the nitrogen flow. The $\mathrm{H}_{2} \mathrm{~S}$ concentrator pressure is no longer a degree of freedom for control of a variable within the $\mathrm{H}_{2} \mathrm{~S}$ concentrator as it has already been used for the control of the $\mathrm{H}_{2} \mathrm{~S}$ purity of the off-gas from the Selexol stripper. For the Selexol stripper, there are no degrees of freedom available for additional controlled variables from it. The three degrees of freedom associated with the stripper are already used for the control of three of the four active constraints: stripper top temperature, stripper pressure, water content of solvent at the bottom of the stripper, and the $\mathrm{H}_{2} \mathrm{~S}$ capture of the process. For the $\mathrm{H}_{2}$ recovery flash vessel, there are two degrees of freedom available: the $\mathrm{H}_{2}$ recovery flash pressure and the semi-lean solvent flow. For the remaining three $\mathrm{CO}_{2}$ flash vessels, the HP flash, MP, and $\mathrm{LP} \mathrm{CO}_{2}$ flash vessels, there is one primary degree of freedom, the respective flash vessel pressures. With the models and constraints required to undertake the optimization defined within Equation 32 calculated and formulated, the optimization problem is solved using the parallelized bi-directional BB algorithm that has been developed. Two disturbances are considered in the optimization. These are changes in the syngas throughput and $\mathrm{CO}_{2}$ capture requirement, as these two disturbances are expected to frequently and continuously affect the process. For this reason, the control system was designed such that near optimal operation will be maintained under these two disturbance conditions, even at the expense of the less frequent and non-sustained disturbances. It should be noted that this formulation does not restrict the number of disturbances considered.

While the parallelized BB algorithm can be solved on a large computer cluster, the current multiobjective optimization problem was run on a dual core $2.6 \mathrm{GHz}$ processor and was solved in approximately 1 hour. The 28 Pareto solutions found from this optimization are shown in Figure 8. However, several of these solutions are only minor variations of one another, e.g., controlling a temperature at the $3^{\text {rd }}$ stage or the $4^{\text {th }}$ stage of a tower. If one examines the solutions that are significantly different from one another, 17 controlled variable sets remain from the original set of 28 . The index of these 17 solutions is shown in Table 7 with the variable corresponding to each index being shown in Table 8. The sets in Table 7 are arranged in descending cost performance and, thus, increasing controllability performance. This means that sets nearer the top of Table 7, and further to the right in Figure 8, are more sensitive to changes in the input variables, implying better control performance. Additionally, sets nearer the bottom 
of Table 6, and further to the left in Figure 8, have better self-optimizing control performance, implying better economic performance.

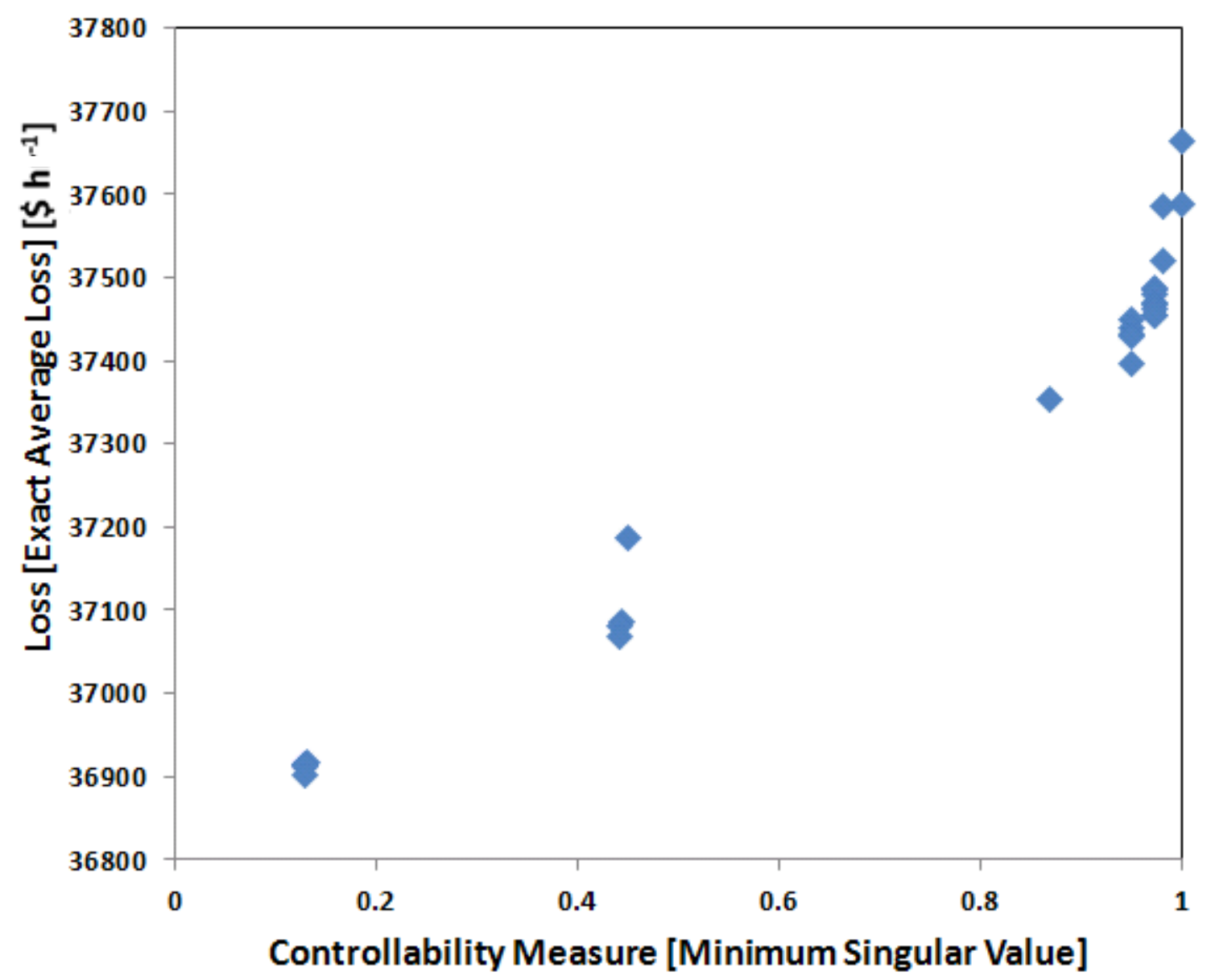

Figure 8. Pareto Solutions for Controlled Variable Selection Problem 
Table 7. Controlled Variable Indices

\begin{tabular}{|c|c|}
\hline Index & Controlled Variable \\
\hline 1 & $\mathrm{H}_{2}$ Recovery Vessel Pressure \\
\hline 2 & High Pressure Vessel Pressure \\
\hline 3 & Medium Pressure Vessel Pressure \\
\hline 4 & Nitrogen Flowrate \\
\hline 5 & Semi-lean Solvent Flowrate \\
\hline 6 & Lean Solvent Flowrate \\
\hline 7 & $\left(\mathrm{y}_{\mathrm{CO} 2}\right)_{8} \mathrm{CO}_{2}$ Absorber $^{\mathrm{a}}$ \\
\hline 8 & $\left(\mathrm{y}_{\mathrm{CO} 2}\right)_{11} \mathrm{CO}_{2}$ Absorber $^{\mathrm{a}}$ \\
\hline 9 & $\left(\mathrm{y}_{\mathrm{CO} 2}\right)_{16} \mathrm{CO}_{2}$ Absorber $^{\mathrm{a}}$ \\
\hline 10 & $\left(\mathrm{x}_{\mathrm{CO} 2}\right)_{8} \mathrm{CO}_{2}$ Absorber $^{\mathrm{a}}$ \\
\hline 11 & $\left(\mathrm{x}_{\mathrm{CO} 2}\right)_{11} \mathrm{CO}_{2}$ Absorber $^{\mathrm{a}}$ \\
\hline 12 & $\mathrm{~T}_{11} \mathrm{CO}_{2}$ Absorber $^{\mathrm{a}}$ \\
\hline 13 & $\left(\mathrm{y}_{\mathrm{CO} 2}\right)_{21} \mathrm{H}_{2} \mathrm{~S}$ Absorber ${ }^{\mathrm{a}}$ \\
\hline 14 & $\left(\mathrm{x}_{\mathrm{CO} 2}\right)_{9} \mathrm{H}_{2} \mathrm{~S}$ Absorber ${ }^{\mathrm{a}}$ \\
\hline 15 & $\left(\mathrm{x}_{\mathrm{H} 2 \mathrm{~S}}\right)_{8} \mathrm{H}_{2} \mathrm{~S}$ Absorber ${ }^{\mathrm{a}}$ \\
\hline 16 & $\left(\mathrm{x}_{\mathrm{H} 2 \mathrm{~S}}\right)_{24} \mathrm{H}_{2} \mathrm{~S}$ Absorber ${ }^{\mathrm{a}}$ \\
\hline 17 & $\left(\mathrm{x}_{\mathrm{H} 2 \mathrm{~S}}\right)_{25} \mathrm{H}_{2} \mathrm{~S}$ Absorber ${ }^{\mathrm{a}}$ \\
\hline 18 & $\left(\mathrm{y}_{\mathrm{CO} 2}\right)_{1} \mathrm{H}_{2} \mathrm{~S}$ Concentrator ${ }^{\mathrm{a}}$ \\
\hline 19 & $\left(\mathrm{y}_{\mathrm{CO} 2}\right)_{4} \mathrm{H}_{2} \mathrm{~S}$ Concentrator ${ }^{\mathrm{a}}$ \\
\hline 20 & $\left(\mathrm{y}_{\mathrm{CO} 2}\right)_{6} \mathrm{H}_{2} \mathrm{~S}$ Concentrator ${ }^{\mathrm{a}}$ \\
\hline 21 & $\left(\mathrm{x}_{\mathrm{CO} 2}\right)_{2} \mathrm{H}_{2} \mathrm{~S}$ Concentrator ${ }^{\mathrm{a}}$ \\
\hline 22 & $\left(\mathrm{x}_{\mathrm{CO} 2}\right)_{6} \mathrm{H}_{2} \mathrm{~S}$ Concentrator ${ }^{\mathrm{a}}$ \\
\hline 23 & $\begin{array}{l}\text { Ratio of Semi-lean:Lean Solvent } \\
\text { Flow to } \mathrm{CO}_{2} \text { Absorber }\end{array}$ \\
\hline 24 & $\begin{array}{l}\text { Ratio of Total Solvent Flow : Gas } \\
\text { Flow to CO2 Absorber }\end{array}$ \\
\hline
\end{tabular}

a. Subscript denote stage number 
Table 8. Controlled Variable Sets Arranged in Descending Cost Performance

\begin{tabular}{|l|l|}
\hline Controlled Variable Sets & Controlled Variable \\
\hline C0 & $1,2,3,4,5,6$ \\
\hline C1 & $2,3,9,20,23,24$ \\
\hline C2 & $2,3,4,9,23,24$ \\
\hline C3 & $1,2,3,4,5,16$ \\
\hline C4 & $1,2,3,10,16,18$ \\
\hline C5 & $1,2,3,4,10,16$ \\
\hline C6 & $1,2,3,7,16,18$ \\
\hline C7 & $1,2,3,4,7,16$ \\
\hline C8 & $1,2,3,7,13,20$ \\
\hline C9 & $1,2,3,4,12,14$ \\
\hline C10 & $1,2,3,4,8,14$ \\
\hline C11 & $1,2,3,4,11,14$ \\
\hline C12 & $1,2,3,17,21,22$ \\
\hline C13 & $2,3,17,21,22,23$ \\
\hline C14 & $2,3,17,19,21,23$ \\
\hline C15 & $2,3,4,5,15,23$ \\
\hline C16 & $2,3,5,15,20,23$ \\
\hline
\end{tabular}

\subsubsection{A Posteriori Analysis}

With the 17 identified candidate controlled variable sets, evaluations using the nonlinear process model are required to finalize the selection. The first nonlinear model evaluation that is undertaken is the economic performance of the controlled variable sets. This is required because the process and the model are nonlinear and the above methods relied upon linearized process models. However, as can be seen within Figures 9 and 10, the economic loss of the process is not linear. This is undertaken within Aspen Plus using the EO solver. With the EO solver within Aspen Plus, it is possible to make specification changes within the system of equations describing the process. In this way, it is possible to fix the controlled variables and to calculate the values of the manipulated variables required to hold the controlled variables constant. With these variable specifications changes implemented for all the controlled variable sets, the disturbances considered are applied and the system of equations is solved. With each magnitude of the disturbances considered, the objective function is evaluated and stored. These evaluations were undertaken for all the controlled variable sets and the results of these examinations are shown in Table 9. If, due to the implementation of the specification changes, no solution exists for the system of equations, the controlled variable set is not feasible as no values of the manipulated variables within the given bounds will be capable of holding the controlled variables at their setpoints. However, it should be noted, that just because no solution exists for a 
controlled variable set, this does not necessarily imply that the controlled variable set is infeasible. For example, controlled variable sets $\mathrm{C} 5, \mathrm{C} 7, \mathrm{C} 9, \mathrm{C} 10$, and $\mathrm{C} 11$ would require a change in the controlled variables when the syngas flow is $75 \%$ lower than the nominal as the $\mathrm{H}_{2} \mathrm{~S}$ concentration in the off-gas from the stripper is no longer an active constraint. Instead, the pressure of the $\mathrm{H}_{2} \mathrm{~S}$ concentrator becomes the new active constraint as the purity of the off-gas becomes greater than what is desired. This does not mean these controlled variable sets are infeasible, simply that at some operating points, the active constraints change. However, other controlled variable sets, for example C15 and C16, are infeasible, as at some operations, specifically when syngas flow is below approximately $90 \%$ of the nominal value, no value of the manipulated variables exist that can hold these controlled variable sets constant while satisfying the process constraints. It is important to note that although the loss of these controlled variable sets appear small, these losses are in terms of dollars per hour. The economic loss of C0, C2, and $\mathrm{C} 10$ at $65 \%$ syngas throughput is $\$ 15,849,000 / \mathrm{yr}, \$ 605,000 / \mathrm{yr}$, and $\$ 815,000 / \mathrm{yr}$, respectively. This calculation is made assuming no down-time in a year.

Table 9. Economic Loss $\left[\$ \mathrm{~h}^{-1}\right]$ of Controlled Variable Sets

\begin{tabular}{|l|l|l|l|}
\hline $\begin{array}{l}\text { Controlled Variable } \\
\text { Set }\end{array}$ & $\begin{array}{l}\text { 65\% Syngas } \\
\text { Throughput }\end{array}$ & $91 \% \mathrm{CO}_{2}$ Capture & $97 \% \mathrm{CO}_{2}$ Capture \\
\hline C0 & 1808 & 88 & 399 \\
\hline C1 & 74 & 62 & 165 \\
\hline C2 & 69 & 62 & 165 \\
\hline C3 & 1159 & 93 & 230 \\
\hline C4 & 101 & 148 & 47 \\
\hline C5 & 106 & 144 & 48 \\
\hline C6 & 101 & 108 & 47 \\
\hline C7 & 106 & 106 & 47 \\
\hline C8 & 92 & 109 & 45 \\
\hline C9 & 93 & 34 & 19 \\
\hline C10 & 93 & 42 & 23 \\
\hline C11 & 93 & 37 & 23 \\
\hline C12 & 138 & 46 & 32 \\
\hline C13 & 56 & 67 & 53 \\
\hline C14 & 209 & & 79 \\
\hline
\end{tabular}




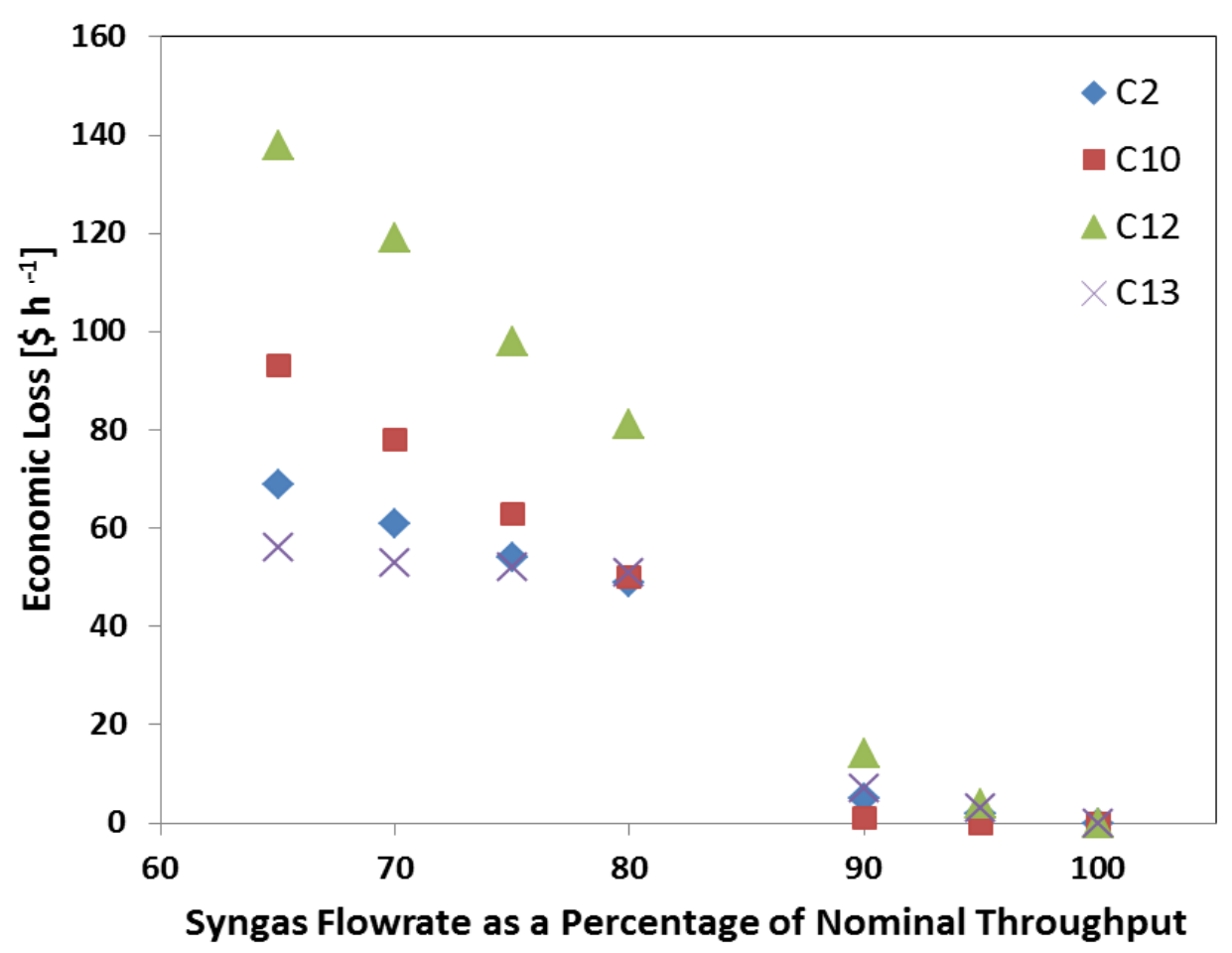

Figure 9. Effect of Syngas Flowrate on Economic Loss of Selected Controlled Variables

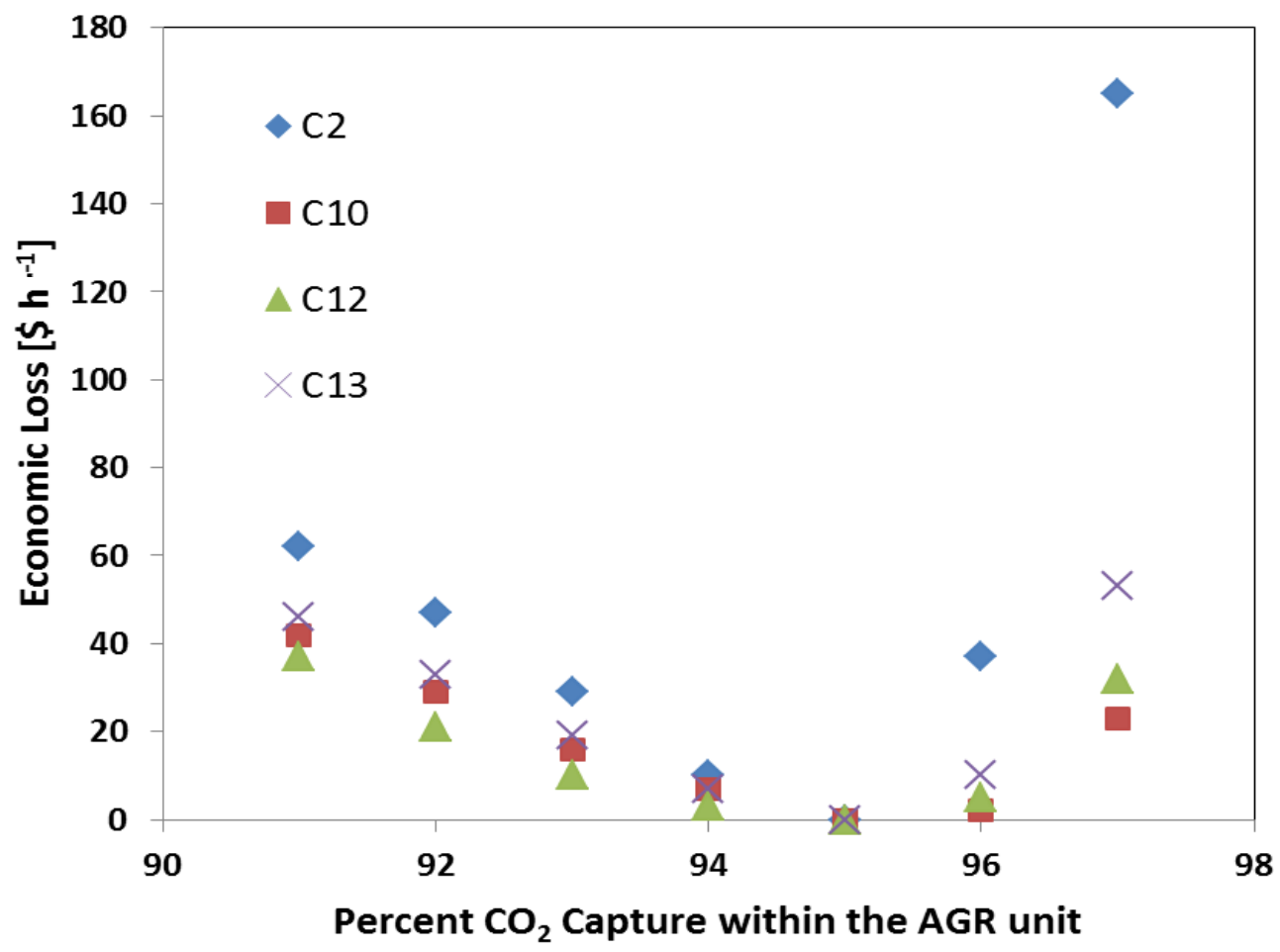

Figure 10. Effect of $\mathrm{CO}_{2}$ Capture Requirement on Economic Loss of Selected Controlled Variables 
With the economic performance of the controlled variable sets determined from the nonlinear model, the control performance must be determined next. This is first done by considering the presence of an RTO. Therefore, the optimal inputs, as determined from the optimization studies, are implemented in the Aspen Plus Dynamics model. From the Aspen Plus Dynamics model, a linear process model of the process is generated, via a CDI script, at that operating point. This is undertaken at various identified optimal operating points of the process. From these linearized process models, the minimum singular value of the appropriately scaled gain matrix is examined for all the feasible controlled variable sets at the extreme disturbance conditions. The results of this analysis are shown in Table 10. From this analysis, it is clear that several of the controlled variable sets lose control performance drastically at the possible extreme possible operations of the process. Also important to note is that the gains of several of the identified controlled variables change direction with deviation from the nominal conditions. As an example, the scaled gain of the lean solvent flow on the $\mathrm{H}_{2} \mathrm{~S}$ content of the solvent on the $24^{\text {th }}$ stage of the $\mathrm{H}_{2} \mathrm{~S}$ absorber is shown in Figure 11 at various syngas flowrates. It should be noted that this inputoutput pairing is suggested by the RGA analysis at nominal operation for all controlled variable sets that contain this particular controlled variable. In general, the controlled variables that are selected from the bottom of the $\mathrm{H}_{2} \mathrm{~S}$ absorber have gain switches associated with them. This is due to the effects of the $\mathrm{H}_{2} \mathrm{~S}$ concentrator pressure and lean solvent flow upon the $\mathrm{H}_{2} \mathrm{~S}$ purity in the stripper off-gas. As lean solvent flow is decreased, the $\mathrm{H}_{2} \mathrm{~S}$ purity in the stripper off-gas increases, causing an increase in the $\mathrm{H}_{2} \mathrm{~S}$ concentrator pressure, resulting in less gas being recycled back to the $\mathrm{H}_{2} \mathrm{~S}$ absorber. Inversely, if lean solvent flow is increased, the $\mathrm{H}_{2} \mathrm{~S}$ purity in the stripper off-gas decreases, causing a decrease in the $\mathrm{H}_{2} \mathrm{~S}$ concentrator pressure, resulting in an increase in gas being recycled back to the $\mathrm{H}_{2} \mathrm{~S}$ absorber. The controlled variables at the bottom of the $\mathrm{H}_{2} \mathrm{~S}$ absorber are sensitive to this recycled flow and also to the lean solvent flow and due to these interactions, the gain of the lean solvent flow may switch on variables at the bottom of the $\mathrm{H}_{2} \mathrm{~S}$ absorber. This does not necessarily imply that the controlled variable sets that contain controlled variables that switch gain are uncontrollable; however, it does imply the need for a more complex control configuration for the control of these controlled variable sets. Additionally, as these gains approach zero, the controllability of the process will, obviously, be sacrificed. 
Table 10. Effect of Disturbances on Controllability of Controlled Variable Sets [minimum singular value]

\begin{tabular}{|l|l|l|l|l|}
\hline $\begin{array}{l}\text { Controlled } \\
\text { Variable Set }\end{array}$ & $\begin{array}{l}\text { Nominal }(95 \% \\
\left.\mathrm{CO}_{2} \text { Capture }\right)\end{array}$ & $\begin{array}{l}\text { 65\% Syngas } \\
\text { Throughput }\end{array}$ & $\begin{array}{l}91 \% \mathrm{CO}_{2} \\
\text { Capture }\end{array}$ & $\begin{array}{l}97 \% \mathrm{CO}_{2} \\
\text { Capture }\end{array}$ \\
\hline $\mathrm{C} 0$ & 1.0000 & 1.0000 & 1.0000 & 1.0000 \\
\hline $\mathrm{C} 1$ & 0.9999 & 0.5672 & 1.0000 & 0.9984 \\
\hline $\mathrm{C} 2$ & 0.9815 & 0.5037 & 0.9884 & 0.9719 \\
\hline $\mathrm{C} 3$ & 0.9815 & 0.6658 & 0.9958 & 0.9496 \\
\hline $\mathrm{C} 4$ & 0.9747 & 0.5278 & 0.9459 & 0.9851 \\
\hline $\mathrm{C} 5$ & 0.9747 & 0.6109 & 0.9459 & 0.9839 \\
\hline $\mathrm{C} 6$ & 0.9745 & 0.5188 & 0.9460 & 0.9840 \\
\hline $\mathrm{C} 7$ & 0.9744 & 0.6071 & 0.9459 & 0.9830 \\
\hline $\mathrm{C} 8$ & 0.9739 & 0.9985 & 0.9456 & 0.9838 \\
\hline $\mathrm{C} 9$ & 0.9513 & 0.9912 & 0.9074 & 0.9728 \\
\hline $\mathrm{C} 10$ & 0.9513 & 0.9906 & 0.9072 & 0.9729 \\
\hline $\mathrm{C} 11$ & 0.9513 & 0.9904 & 0.9071 & 0.9729 \\
\hline $\mathrm{C} 12$ & 0.8698 & 0.2757 & 0.1657 & 0.9294 \\
\hline $\mathrm{C} 13$ & 0.4499 & 0.1551 & 0.4554 & 0.3858 \\
\hline
\end{tabular}

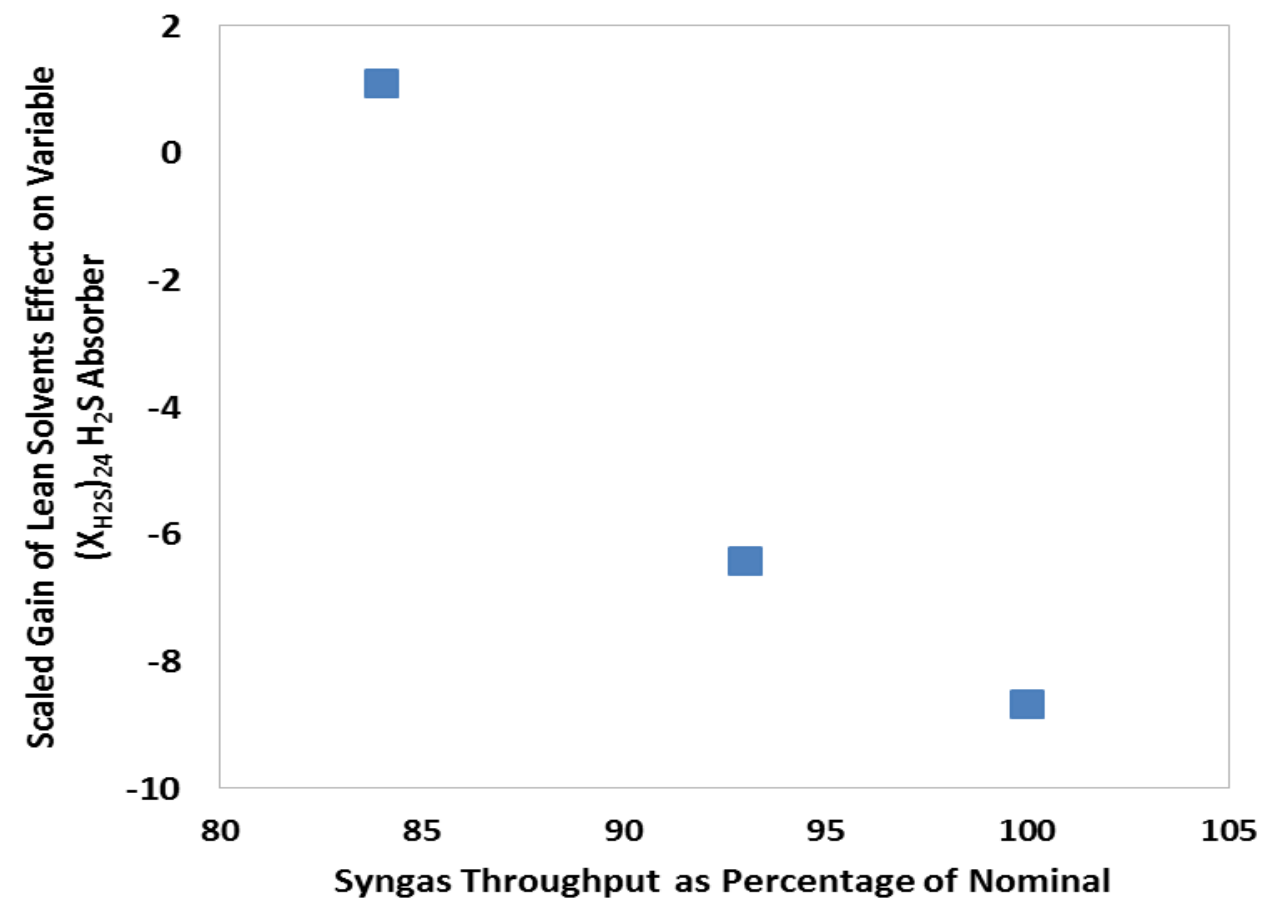

Figure 11. Effect of Syngas Throughput on the Gain of Controlled Variable With these analyses complete, the controlled variable sets $\mathrm{C} 9, \mathrm{C} 10$, and $\mathrm{C} 11$ appear to be the superior sets, in terms of both controllability and economics, and are thus selected for further consideration. At this point, further analysis can be performed to reduce the size of the controlled variable sets. C11 contains a variable associated with the solvent composition within the $\mathrm{H}_{2} \mathrm{~S}$ absorber. In comparison, controlled variable sets $\mathrm{C} 9$ and $\mathrm{C} 10$ contain a variable 
associated with temperature and a vapor composition. With the exception of these differences, these controlled variable sets are the same. As the measurement of liquid compositions is more expensive and more susceptible to errors, the selection of a vapor phase composition or temperature would be preferred. Given these process insights, the controlled variable sets C9 and $\mathrm{C} 10$ are considered for further evaluation.

To determine the controllability of controlled variable sets $\mathrm{C} 9$ and $\mathrm{C} 10$, the maximum magnitude of a disturbance is applied to the model in the absence of a RTO. This is undertaken for all disturbances. The process is then linearized at the new operational point, yielding six linearized models of the process. The gain matrices generated are then scaled and the minimum singular values are calculated. This provides insight into the control performance of these controlled variables when either no RTO is present or no updated setpoints from the RTO is available. The results of these analyses are shown in Table 11. These results show that even if no RTO is present or no updated setpoints from an RTO are available the controllability of controlled variable sets C9 and C10 are still satisfactory. For these reasons, controlled variable sets C9 and C10 may be considered as primary controlled variable sets for the design of the control system of the AGR unit as part of an IGCC power plant. For future evaluations, controlled variable set C10 is considered.

Table 11. Controllability [minimum singular value] of C9 and C10 with Constant Setpoint Policy

\begin{tabular}{|l|c|c|}
\hline Disturbance & $\mathrm{C} 9$ & $\mathrm{C} 10$ \\
\hline $65 \%$ Syngas Throughput & 0.9997 & 0.9718 \\
\hline $91 \% \mathrm{CO}_{2}$ Capture & 0.9665 & 0.9314 \\
\hline $97 \% \mathrm{CO}_{2}$ Capture & 0.9312 & 0.9665 \\
\hline
\end{tabular}

\subsubsection{Bottom-Up Design}

In this section, the selection of secondary controlled variables, control structure of the supervisory control layer, and the design of the MPCs used for control of the AGR unit will be discussed.

\subsubsection{Secondary Controlled Variable Selection}

\subsection{A Priori Analysis}

To begin the analysis, a set of candidate secondary controlled variables needs to be identified. For this application, the set of secondary controlled variables is the same as previously used for the primary controlled variable selection, shown in Table 12, unless they were selected as 
primary controlled variables. It should be noted that one would not usually include compositions as candidate secondary controlled variables because of time-delay in the measurement. However, as not all such variables are evident in a process unit, the motivation for including the composition variables in the candidate set of sensors is to see the efficacy of the proposed algorithm in removing them. Next, local disturbances likely to affect the process are identified. These are listed in Table 13. 
Table 12. Candidate Set of Secondary Controlled Variables

\begin{tabular}{|c|c|}
\hline Controlled Variable & $\begin{array}{c}\text { Number of Controlled } \\
\text { Variable }\end{array}$ \\
\hline Temperature of stages in $\mathrm{CO}_{2}$ Absorber & 16 \\
\hline $\mathrm{H}_{2} \mathrm{~S}$ vapor fraction of stages in $\mathrm{CO}_{2}$ Absorber & 15 \\
\hline $\mathrm{CO}_{2}$ vapor fraction of stages in $\mathrm{CO}_{2}$ Absorber & 15 \\
\hline $\mathrm{H}_{2} \mathrm{~S}$ liquid fraction of stages in $\mathrm{CO}_{2}$ Absorber & 16 \\
\hline $\mathrm{CO}_{2}$ liquid fraction of stages in $\mathrm{CO}_{2}$ Absorber & 16 \\
\hline Temperature of stages in $\mathrm{H}_{2} \mathrm{~S}$ Absorber & 27 \\
\hline $\mathrm{H}_{2} \mathrm{~S}$ vapor fraction of stages in $\mathrm{H}_{2} \mathrm{~S}$ Absorber & 27 \\
\hline $\mathrm{CO}_{2}$ vapor fraction of stages in $\mathrm{H}_{2} \mathrm{~S}$ Absorber & 27 \\
\hline $\mathrm{H}_{2} \mathrm{~S}$ liquid fraction of stages in $\mathrm{H}_{2} \mathrm{~S}$ Absorber & 27 \\
\hline $\mathrm{CO}_{2}$ liquid fraction of stages in $\mathrm{H}_{2} \mathrm{~S}$ Absorber & 27 \\
\hline Temperature of $\mathrm{H}_{2}$ Recovery Flash & 1 \\
\hline $\mathrm{H}_{2}$ vapor fraction of $\mathrm{H}_{2}$ Recovery Flash & 1 \\
\hline $\mathrm{CO}_{2}$ vapor fraction of $\mathrm{H}_{2}$ Recovery Flash & 1 \\
\hline $\mathrm{H}_{2}$ liquid fraction of $\mathrm{H}_{2}$ Recovery Flash & 1 \\
\hline $\mathrm{CO}_{2}$ liquid fraction of $\mathrm{H}_{2}$ Recovery Flash & 1 \\
\hline Temperature of HP Flash & 1 \\
\hline $\mathrm{H}_{2}$ vapor fraction of HP Flash & 1 \\
\hline $\mathrm{CO}_{2}$ vapor fraction of HP Flash & 1 \\
\hline $\mathrm{H}_{2}$ liquid fraction of HP Flash & 1 \\
\hline $\mathrm{CO}_{2}$ liquid fraction of HP Flash & 1 \\
\hline Temperature of MP Flash & 1 \\
\hline $\mathrm{H}_{2}$ vapor fraction of MP Flash & 1 \\
\hline $\mathrm{CO}_{2}$ vapor fraction of MP Flash & 1 \\
\hline $\mathrm{H}_{2}$ liquid fraction of MP Flash & 1 \\
\hline $\mathrm{CO}_{2}$ liquid fraction of MP Flash & 1 \\
\hline Temperature of LP Flash & 1 \\
\hline $\mathrm{H}_{2}$ vapor fraction of LP Flash & 1 \\
\hline $\mathrm{CO}_{2}$ vapor fraction of LP Flash & 1 \\
\hline $\mathrm{H}_{2}$ liquid fraction of LP Flash & 1 \\
\hline $\mathrm{CO}_{2}$ liquid fraction of LP Flash & 1 \\
\hline Temperature of stages in $\mathrm{H}_{2} \mathrm{~S}$ Concentrator & 6 \\
\hline $\mathrm{H}_{2} \mathrm{~S}$ vapor fraction of stages in $\mathrm{H}_{2} \mathrm{~S}$ Concentrator & 6 \\
\hline $\mathrm{CO}_{2}$ vapor fraction of stages in $\mathrm{H}_{2} \mathrm{~S}$ Concentrator & 6 \\
\hline $\mathrm{H}_{2} \mathrm{~S}$ liquid fraction of stages in $\mathrm{H}_{2} \mathrm{~S}$ Concentrator & 6 \\
\hline $\mathrm{CO}_{2}$ liquid fraction of stages in $\mathrm{H}_{2} \mathrm{~S}$ Concentrator & 6 \\
\hline Temperature of stages in Selexol Stripper & 9 \\
\hline $\mathrm{H}_{2} \mathrm{~S}$ vapor fraction of stages in Selexol Stripper & 10 \\
\hline $\mathrm{CO}_{2}$ vapor fraction of stages in Selexol Stripper & 11 \\
\hline $\mathrm{H}_{2} \mathrm{~S}$ liquid fraction of stages in Selexol Stripper & 11 \\
\hline $\mathrm{CO}_{2}$ liquid fraction of stages in Selexol Stripper & 11 \\
\hline Split Fraction of Solvent at Bottom of $\mathrm{CO}_{2}$ Absorber & 1 \\
\hline Ratio of Syngas - Solvent inlets to $\mathrm{CO}_{2}$ Absorber & 1 \\
\hline
\end{tabular}


Table 13. Identified Disturbances

\begin{tabular}{|l|c|}
\hline \multicolumn{1}{|c|}{ Disturbance } & Disturbance Magnitude \\
\hline Water Cooler Outlet Temperatures & $+16.7{ }^{\circ} \mathrm{C}$ \\
\hline Refrigeration Cooler Outlet Temperatures & $+2.8{ }^{\circ} \mathrm{C}$ \\
\hline Syngas Composition (percent deviation in & ${ }^{y}{ }^{2}= \pm 20 \%$ \\
mol fraction) & ${ }^{y}{ }_{\mathrm{H}_{2}}= \pm 0.36 \%$ \\
& ${ }^{y_{C O}}= \pm 0.76 \%$ \\
& ${ }^{y_{H_{2} O}}= \pm 0.38 \%$ \\
\hline $\mathrm{H}_{2} \mathrm{~S}$ Flowrate in the Syngas & $\pm 35 \mathrm{kmols} \mathrm{h}^{-1}$ \\
\hline
\end{tabular}

With the candidate set and disturbance variables identified, linearized process models are calculated from the nonlinear Aspen Plus Dynamics ${ }^{\circledR}$ model using a control design interface (CDI) script. Using the CDI script, the linearized process gain matrices for the primary and secondary controlled variables, $G_{p 1}$ and $G_{p 2}$, respectively, and disturbance gain matrices for the primary and secondary controlled variables, $G_{d 1}$ and $G_{d 2}$, respectively, are calculated. Using these matrices, Equations 1 and 2 are used to formulate the subset selection constraint.

Next, the identification of input-output models must be completed. To accomplish this, the nonlinear process model is perturbed from its steady-state, nominal operating conditions. Perturbations are applied to the inputs of interest, listed in Table 14 and the disturbances defined in Table 15. The outputs of the primary and candidate secondary controlled variables are stored. With this data, transfer functions are identified within MATLAB ${ }^{\circledR}$. Any number of forms of the transfer function can be considered. For this application, three forms are fitted in MATLAB ${ }^{\circledR}$. The first is a simple first-order model plus time-delay, Equation 48. Here, $\Theta_{p}$ is the process dead-time and $\tau$ is the process time constant. The second is a second-order model plus timedelay, Equation 49. Here, $\Theta_{p}$ is the process dead-time, $\omega_{n}$ is the undamped natural frequency, and $\zeta$ is the damping ratio. The third is a first-order model plus pure gain, Equation 50. Here, $\tau$ is the process time constant and $\alpha$ is the fraction of the total gain that applies instantaneously. Also, for all these transfer functions, $K$ is the gain. Within MATLAB, the parameters of these equations are used as degrees of freedom to minimize the sum of squared errors between the model output and the output from the nonlinear process model. Next, the Akaike information criterion (AIC) (Akaike,1974) is calculated for each of the models, defined as Equation 51, and the model which has the lowest value is used for the next stage of analysis. Here, TSE is the total 
squared error, $n_{\text {data }}$ is the number of data points, and $n_{p}$ is the number of fitting parameters associated with the model.

Table 14. Manipulated Variables for Control of Secondary Controlled Variables

\begin{tabular}{|l|}
\hline Low Pressure Flash Vessel Pressure \\
\hline Temperature at Bottom of Stripper \\
\hline Steam Flowrate to Stripper \\
\hline $\mathrm{H}_{2} \mathrm{~S}$ Concentrator Pressure \\
\hline Semi-lean Solvent Flowrate \\
\hline Lean Solvent Flowrate \\
\hline
\end{tabular}

Table 15. Disturbance Variables Considered during Secondary Controlled Variable Selection

\begin{tabular}{|l|c|}
\hline \multicolumn{1}{|c|}{ Disturbance } & Disturbance Magnitude \\
\hline Water Cooler Outlet Temperatures & $+16.7^{\circ} \mathrm{C}$ \\
\hline Refrigeration Cooler Outlet Temperatures & $+2.8^{\circ} \mathrm{C}$ \\
\hline Syngas Composition (extent of WGS reaction) & $\pm 272 \mathrm{kmols}$ \\
\hline $\mathrm{H}_{2} \mathrm{~S}$ Content of Syngas & $\pm 35 \mathrm{kmols} \mathrm{h}^{-1}$ \\
\hline
\end{tabular}

$$
\begin{gathered}
g_{1}(s)=\frac{K e^{-\Theta_{p} s}}{\tau^{-1} s+1} \\
g_{2}(s)=\frac{K \omega_{n}^{2} e^{-\Theta_{p} s}}{s^{2}+2 \xi \omega_{n} s+\omega_{n}^{2}} \\
g_{3}(s)=\frac{K(\alpha s+\tau)}{s+\tau} \\
A I C=n_{\text {data }} \ln \left(\frac{T S E}{n_{\text {data }}}\right)+2 n_{p}
\end{gathered}
$$

Finally, the IAEs of all feasible pairings, as determined by the subset selection constraint, of $u-$ $y_{2}-y_{1}$ are calculated. For the dynamics of the measurement devices, pure time-delay is assumed. The time-delay applied is based upon the type of measurement. If the secondary controlled variable is a temperature, flowrate, or pressure, it is assumed that there is no timedelay. For vapor compositions, measurement delay was assumed to be $2 \mathrm{~min}$. For liquid compositions, measurement delay was assumed to be $5 \mathrm{~min}$. In addition, the IAE calculations rely upon the design of the controllers $G_{C 1}$ and $G_{C 2}$. For this application, both controllers are assumed to be PI controllers and are tuned using the Ciancone correlations (Ciancone and 
Marlin, 1990). This tuning method is defined in Tables 16 and 17. For a PI controller of the form $K_{C}\left(1+\frac{1}{\tau_{I} s}\right)$, the controller gain, $K_{C}$, is determined from Table 16, and the integral time constant $\tau_{I}$, is determined from Table 17. First, the inner, secondary, controller is designed, $G_{C 2}$. This is done by providing a step to $G_{2} H_{2}$ and fitting the response to a first-order system plus time-delay. From this, Tables 5 and 6 are used to design the controller. For the design of the outer, primary, controller, $G_{C 1}$, a step is provided to $\frac{G_{C 2} G_{1} H_{1}}{1+G_{C 2} G_{2} H_{2}}$ and the response is fitted to a first-order system plus time-delay. Again, like with the secondary controller, Tables 16 and 17 are used to design the controller. The results of all of these calculations are stored in a three dimensional matrix which is used during set selection.

Table 16. Controller Gain Table

\begin{tabular}{|l|l|l|l|l|l|l|l|l|l|}
\hline$\frac{\Theta_{p}}{\tau+\Theta_{p}}$ & 0.0 & 0.1 & 0.2 & 0.3 & 0.4 & 0.5 & 0.6 & 0.7 & 0.8 \\
\hline$K K c$ & 1.1 & 1.1 & 1.8 & 1.1 & 1.0 & 0.8 & 0.54 & 0.42 & 0.32 \\
\hline
\end{tabular}

Table 17. Integral Time Constant Table

\begin{tabular}{|l|l|l|l|l|l|l|l|l|l|}
\hline$\frac{\Theta_{p}}{\tau+\Theta_{p}}$ & 0.0 & 0.1 & 0.2 & 0.3 & 0.4 & 0.5 & 0.6 & 0.7 & 0.8 \\
\hline$\frac{\tau_{I}}{\tau+\Theta_{p}}$ & 0.23 & 0.23 & 0.23 & 0.72 & 0.72 & 0.70 & 0.67 & 0.60 & 0.53 \\
\hline
\end{tabular}

\subsection{Set Selection}

With the a priori analysis complete, the parallelized, bi-directional branch and bound algorithm described Appendix A is used to solve the constrained mixed integer optimization problem. Two cases are considered here, the first has no constraint on the servo control performance of the supervisory control layer. In the second, the servo control constraint on the supervisory control layer may only deteriorate the performance by $20 \%$ from the case with no secondary controlled variables. That is $\varepsilon=1.2\left(\operatorname{IAE}_{\text {SERVO }}\right)_{\text {basis }}$, as defined in Equation 40, where $\left(I A E_{\text {SERVO }}\right)_{\text {basis }}$ is the 
IAE value of the closed loop system with no secondary controlled variables. The deterioration of the servo control performance was set to $20 \%$ from the case with no secondary controlled variables because if the constraint is tighter no solutions exist and if looser does not change the solution set. The scaling applied to the servo IAEs are the same as used for the regulatory IAEs. For both of these cases, ten solutions were desired; however, for Case 2, because of the servo constraint, the fifth best solution is the trivial case where no secondary controlled variables are selected. This means that all solutions that follow the fifth solution in Case 2, though not violating any constraints, result in a deterioration of control performance from the case with no secondary controlled variables. Therefore, only five solutions were found for Case 2 that did not result in worse control performance from the base case. It should be noted that as this is a single objective optimization, the user must specify the number of solutions desired. However, if a large number of solutions are desired, the computation time required for solving the optimization problem will become high. However, enough solutions should be acquired to ensure that a viable solution is found after completing the posteriori analysis. The results of Cases 1 and 2 are shown in Tables 18 and 19, respectively, where the indices of the controlled variables are shown in Table 20. It should be noted that little difference exists in the solutions found in Case 1; however, more substantial differences between the solutions exist within Case 2. For this application, no servo constraint is required because the setpoints of the primary controlled variables are not expected to change and, therefore, the remaining discussions will focus on Case 1. Within the solutions in Case 1, there are two categories of sets that were observed. Each of these categories are outlined within Table 21. The first category, containing sets 1, 2, 3, 4, 5, 6, 7, and 9, use the LP flash vessel pressure to control the $\mathrm{CO}_{2}$ capture, via a secondary controlled variable, and the second category, containing Sets 8 and 10, use the semi-lean solvent flowrate, via a secondary controlled variable, to control the $\mathrm{CO}_{2}$ capture. Another important distinction between these two categories is related to the primary controlled variables whose regulatory control performance is improved. In the case of the first category, the primary controlled variables whose performance is improved are $\mathrm{CO}_{2}$ capture and solvent composition in the $\mathrm{H}_{2} \mathrm{~S}$ absorber. The improvements in $\mathrm{CO}_{2}$ capture and solvent composition in the $\mathrm{H}_{2} \mathrm{~S}$ absorber are $69 \%$ and $48 \%$ reductions in the IAE values, respectively. $\mathrm{CO}_{2}$ capture control, for all ten secondary controlled variable sets, is a controlled variable whose performance is always improved upon. This is due to the large economic role that the $\mathrm{CO}_{2}$ capture plays within the AGR unit. Therefore, secondary controlled variables are found that will provide superior 
performance in $\mathrm{CO}_{2}$ capture. The solvent composition within the $\mathrm{H}_{2} \mathrm{~S}$ absorber, although it does not have as large of an impact on the economics of the process as other primary controlled variables, does suffer from poor control performance. This is due to the large distance, and thus large dead-time, of the manipulated variable, lean solvent flow, from the controlled variable. Additionally, as this primary controlled variable is a liquid composition, measurement delays also contribute to the poor control performance. Therefore, the controlled variable sets associated with the first category seek to improve both the control performance of the $\mathrm{CO}_{2}$ capture and the solvent composition of the $\mathrm{H}_{2} \mathrm{~S}$ absorber. However, in the case of the controlled variables associated with the second category, only $\mathrm{CO}_{2}$ capture loop performance is improved. The improvements in $\mathrm{CO}_{2}$ capture for this category result in a $99 \%$ reduction in the IAE value. In this case, the secondary controlled variables result in a restructuring of the ultimate pairing of manipulated variable to primary controlled variables. The result is a structure that provides superior control performance improvements of the $\mathrm{CO}_{2}$ capture than that used within the second category. In this case, however, there are no secondary controlled variables selected to improve the control performance associated with the control of the $\mathrm{H}_{2} \mathrm{~S}$ absorber composition.

Table 18. Results of optimization with no constraint on servo performance

\begin{tabular}{|c|c|l|l|l|l|l|l|}
\hline $\begin{array}{c}\text { Objective Function } \\
\text { Value }\end{array}$ & $\begin{array}{c}\text { Set } \\
\text { ID }\end{array}$ & \multicolumn{6}{|c|}{ Secondary Controlled Variables } \\
\hline 16.08034 & 1 & 1 & 2 & 3 & 4 & 5 & 6 \\
\hline 16.10541 & 2 & 1 & 2 & 4 & 5 & 6 & 7 \\
\hline 16.12042 & 3 & 1 & 2 & 4 & 5 & 6 & 8 \\
\hline 16.17628 & 4 & 1 & 2 & 4 & 5 & 6 & 9 \\
\hline 16.26826 & 5 & 1 & 3 & 4 & 5 & 6 & 10 \\
\hline 16.29332 & 6 & 1 & 4 & 5 & 6 & 7 & 10 \\
\hline 16.29506 & 7 & 1 & 2 & 4 & 5 & 6 & 11 \\
\hline 16.33530 & 8 & 5 & 9 & 10 & 12 & 13 & 16 \\
\hline 16.33962 & 9 & 1 & 4 & 5 & 6 & 7 & 18 \\
\hline 16.36419 & 10 & 5 & 6 & 7 & 10 & 13 & 16 \\
\hline
\end{tabular}


Table 19. Results of optimization with constraint on servo performance

\begin{tabular}{|c|l|l|l|l|l|l|}
\hline $\begin{array}{c}\text { Objective } \\
\begin{array}{c}\text { Function } \\
\text { Value }\end{array}\end{array}$ & \multicolumn{7}{|c|}{$\begin{array}{c}\text { Secondary Controlled Variables } \\
\text { Indices }\end{array}$} \\
\hline 16.60080 & 1 & 4 & 5 & 6 & 10 & 13 \\
\hline 16.79399 & 4 & 5 & 6 & 10 & 13 & 17 \\
\hline 26.46106 & 4 & 5 & 6 & 13 & 14 & 16 \\
\hline 26.60726 & 4 & 5 & 6 & 13 & 14 & 15 \\
\hline 26.67772 & 4 & 5 & 6 & 10 & 13 & 14 \\
\hline
\end{tabular}

Table 20. Controlled variables indices

\begin{tabular}{|c|c|}
\hline Index & Controlled Variables \\
\hline 1 & $\left(\mathrm{x}_{\mathrm{CO} 2}\right)_{6} \mathrm{CO}_{2}$ Absorber $^{\mathrm{a}}$ \\
\hline 2 & $\mathrm{~T}_{1} \mathrm{H}_{2} \mathrm{~S}$ Absorber ${ }^{\mathrm{a}}$ \\
\hline 3 & $\left(\mathrm{y}_{\mathrm{CO} 2}\right)_{1} \mathrm{H}_{2} \mathrm{~S}_{\text {Concentrator }}{ }^{\mathrm{a}}$ \\
\hline 4 & $\mathrm{~T}_{11}$ Selexol Stripper ${ }^{\mathrm{a}}$ \\
\hline 5 & Steam Flowrate to Stripper \\
\hline 6 & $\mathrm{H}_{2} \mathrm{~S}$ Concentrator Operating Pressure \\
\hline 7 & $\left(\mathrm{y}_{\mathrm{CO} 2}\right)_{25} \mathrm{H}_{2} \mathrm{~S}$ Absorber ${ }^{\mathrm{a}}$ \\
\hline 8 & $\left(\mathrm{y}_{\mathrm{CO} 2}\right)_{6} \mathrm{H}_{2} \mathrm{~S}_{\text {Absorber }}{ }^{\mathrm{a}}$ \\
\hline 9 & $\mathrm{~T}_{22} \mathrm{H}_{2} \mathrm{~S}$ Absorber ${ }^{\mathrm{a}}$ \\
\hline 10 & Semi-lean Solvent Flowrate \\
\hline 11 & $\left(\mathrm{y}_{\mathrm{H} 2 \mathrm{~S}}\right)_{20} \mathrm{H}_{2} \mathrm{~S}$ Absorber ${ }^{\mathrm{a}}$ \\
\hline 12 & $\left(\mathrm{x}_{\mathrm{CO} 2}\right)_{6} \mathrm{H}_{2} \mathrm{~S}_{\text {Concentrator }}^{\mathrm{a}}$ \\
\hline 13 & Lean Solvent Flowrate \\
\hline 14 & LP Flash Vessel Pressure \\
\hline 15 & $\left(\mathrm{x}_{\mathrm{CO} 2}\right)_{3} \mathrm{H}_{2} \mathrm{~S}$ Absorber ${ }^{\mathrm{a}}$ \\
\hline 16 & $\mathrm{~T}_{16} \mathrm{CO}_{2}$ Absorber ${ }^{\mathrm{a}}$ \\
\hline 17 & $\left(\mathrm{y}_{\mathrm{CO} 2}\right)_{6} \mathrm{CO}_{2}$ Absorber $^{\mathrm{a}}$ \\
\hline 18 & $\left(\mathrm{x}_{\mathrm{H} 2 \mathrm{~S}}\right)_{6} \mathrm{H}_{2} \mathrm{~S}$ Concentrator ${ }^{\mathrm{a}}$ \\
\hline
\end{tabular}

a. Subscript denote stage number 
Table 21. Ultimate Pairings of Manipulated Variables to Primary Controlled for each Category of Secondary Controlled Variable

\begin{tabular}{|l|l|l|l|}
\hline Category 1 & Category 2 \\
\hline Manipulated Variable & Controlled Variable & Manipulated Variable & Controlled Variable \\
\hline $\begin{array}{l}\text { LP Flash Vessel } \\
\text { Pressure }\end{array}$ & $\mathrm{CO}_{2}$ Capture & $\begin{array}{l}\text { Semi-lean Solvent } \\
\text { Flowrate }\end{array}$ & $\mathrm{CO}_{2}$ Capture \\
\hline $\mathrm{T}_{11}$ Selexol Stripper & \\
\hline $\begin{array}{l}\text { Steam Flowrate to } \\
\text { Stripper }\end{array}$ & $\begin{array}{l}\mathrm{H}_{2} \mathrm{~S} \text { Capture } \\
\text { Sater Content of }\end{array}$ & $\begin{array}{l}\text { Steam Flowrate to } \\
\text { Stripper }\end{array}$ & $\begin{array}{l}\text { Water Content of } \\
\text { Solvent }\end{array}$ \\
\hline $\begin{array}{l}\mathrm{H}_{2} \mathrm{~S} \text { Concentrator } \\
\text { Operating Pressure }\end{array}$ & $\mathrm{H}_{2} \mathrm{~S}$ Purity to Claus & $\begin{array}{l}\mathrm{H}_{2} \mathrm{~S} \text { Concentrator } \\
\text { Operating Pressure }\end{array}$ & $\mathrm{H}_{2} \mathrm{~S}$ Purity to Claus \\
\hline $\begin{array}{l}\text { Semi-lean Solvent } \\
\text { Flowrate }\end{array}$ & $\begin{array}{l}\text { Vapor Composition in } \\
\mathrm{CO}_{2} \text { Absorber }\end{array}$ & $\begin{array}{l}\text { LP Flash Vessel } \\
\text { Pressure }\end{array}$ & $\begin{array}{l}\text { Vapor Composition in } \\
\mathrm{CO}_{2} \text { Absorber }\end{array}$ \\
\hline $\begin{array}{l}\text { Lean Solvent } \\
\text { Flowrate }\end{array}$ & $\begin{array}{l}\text { Solvent Composition } \\
\text { in } \mathrm{H}_{2} \mathrm{~S} \text { Absorber }\end{array}$ & $\begin{array}{l}\text { Lean Solvent } \\
\text { Flowrate }\end{array}$ & $\begin{array}{l}\text { Solvent Composition } \\
\text { in } \mathrm{H}_{2} \mathrm{~S} \text { Absorber }\end{array}$ \\
\hline
\end{tabular}

a. Subscript denote stage number

\subsection{Posteriori Analysis}

With the set selection step of the procedure complete, the posteriori analysis is required to finalize the selection of the secondary controlled variable set. This includes an analysis of the regulatory and servo control performance of the regulatory control layer at off-design operating conditions. These off-design operations are $65 \%$ throughput, $91 \% \mathrm{CO}_{2}$ capture, and $97 \% \mathrm{CO}_{2}$ capture. These operations were identified in Section 4.2.1.1.1 and are used here for the analysis of the performance of the secondary controlled variable sets. The measure for these control performances are the minimum singular value of the process gain matrix for a measure of the servo control performance, and the maximum singular value of $G_{d 2} G_{p 2}^{-1}$ for a measure of the regulatory control performance. The requirement is that the servo control performance measure should be greater than 1 and the regulatory control measure should be less than 1 . The results of this off-design analysis are shown in Table 4. These are the same constraints applied during the set selection procedure; however, in that instance the constraint is applied only at nominal operations. As can be seen in Table 22, the only controlled variable sets that satisfy all the constraints under off-design operation are sets 5, 6, and 9. Finally, these sets need to be examined to ensure that there is no gain switching that occurs within the regulatory control layer. None of these sets are found to contain pairings whose gains will switch in the operational regions examined. 
Table 22. Off-design control performance regulatory control layer

\begin{tabular}{|l|l|l|l|l|l|l|}
\hline \multirow{2}{*}{$\begin{array}{l}\text { Controlled } \\
\text { Variable Set }\end{array}$} & \multicolumn{4}{|l|}{} & \multicolumn{2}{l|}{$91 \% \mathrm{CO}_{2}$} \\
\cline { 2 - 7 } & $65 \%$ Throughput & \multicolumn{2}{l|}{\begin{tabular}{l}
\multicolumn{2}{l|}{$\mathrm{CO}_{2}$ Capture } \\
Capture
\end{tabular}} \\
\cline { 2 - 7 } & Servo & Regulatory & Servo & Regulatory & Servo & Regulatory \\
\hline 1 & 2.3014 & 0.8788 & 1.1242 & 2.351 & 2.896 & 0.9686 \\
\hline 2 & 2.7322 & 0.9164 & 1.1309 & 2.3382 & 2.9781 & 0.9868 \\
\hline 3 & 2.6676 & 0.9392 & 1.1327 & 2.3345 & 2.9489 & 1.0041 \\
\hline 4 & 2.5456 & 0.9573 & 1.1300 & 2.3787 & 2.6538 & 0.9735 \\
\hline 5 & 2.8695 & 0.0087 & 3.8224 & 0.0084 & 3.1984 & 0.0121 \\
\hline 6 & 2.8239 & 0.0015 & 7.0058 & 0.0012 & 3.2608 & 0.0073 \\
\hline 7 & 0.3451 & 1.338 & 1.1211 & 2.3577 & 2.8434 & 0.98 \\
\hline 8 & 0.4412 & 13.4544 & 5.6637 & 0.6574 & 1.0577 & 18.0605 \\
\hline 9 & 2.7803 & 0.0007877 & 11.081 & 0.0005928 & 2.7666 & 0.0087 \\
\hline 10 & 1.5753 & 3.3046 & 42.564 & 0.1283 & 3.9609 & 2.8914 \\
\hline
\end{tabular}

\subsubsection{Control Structure Design}

In this section, it will be discussed how the optimal control structure of the supervisory control layer of the AGR unit was determined. To begin, the linear state space model of the AGR unit was required. This was obtained from Aspen Plus Dynamics using the CDI script. From this state space model, the controllability and observability Gramians are calculated for each of the individual subsystems, i.e., each of the pairings of input to output. From these calculations, the three unscaled Gramian interaction matrices are attained. Next, each of these Gramian interaction matrices are scaled, according to the methodology discussed in Section 3.3.1.

With these matrices, we determine the optimal control structure for the process. To accomplish this, the optimal pairings of inputs and outputs are determined for both centralized and decentralized control structures. One of the three Gramian interaction matrices is used to determine the optimal pairings of the structure. These Gramian interaction measures may lead to the same or different control structures. However, it is important to note that the Gramian interaction measures will sometimes suggest unstable structures for a decentralized control case. Therefore, a constraint is imposed on the optimization that ensures that the pairings used for the decentralized controls are always in agreement with those suggested by the RGA. The optimization is solved for all possible control structures that involve either decentralized or centralized, or any combination thereof. With these results, it is possible to determine the expected control performance of each of the possible structures. Next, the controller complexity 
for each of these structures is determined and with that a Pareto set of possible control structures is obtained. Using these measures for control performance and control complexity, the optimization problem shown as Equation 52 is solved where $J_{\text {control }}$ is calculated from one of the Gramian interaction measures. The results of these optimization are shown in Figures 12, 13, and 14 for the HIIA, PM and $\Sigma_{2}$ interactions measures, respectively. To determine the actual structure to be used the numerical derivative of the control performance criteria with respect to the controller complexity is calculated for the HIIA, PM and $\Sigma_{2}$ interactions measures, listed in Table 23. From this tables, it can be seen that a large change in expected control performance improvement with increasing controller complexity is seen for the case beyond one 4 by 4 and one 2 by 2 centralized controllers. Therefore, for this application, that is the control structure that was used.

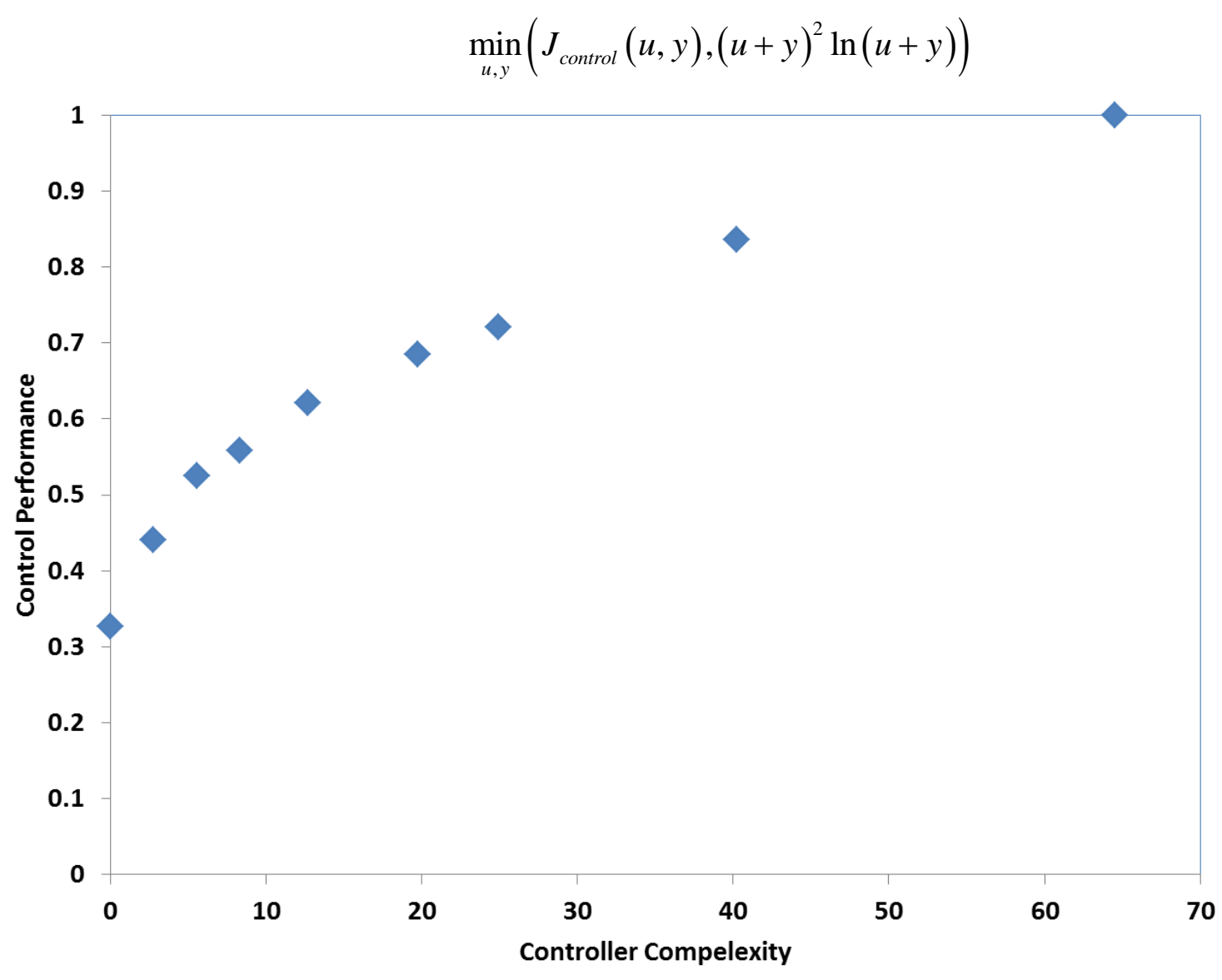

Figure 12. Pareto Set for Control Structure using HIIA Interaction Measure 


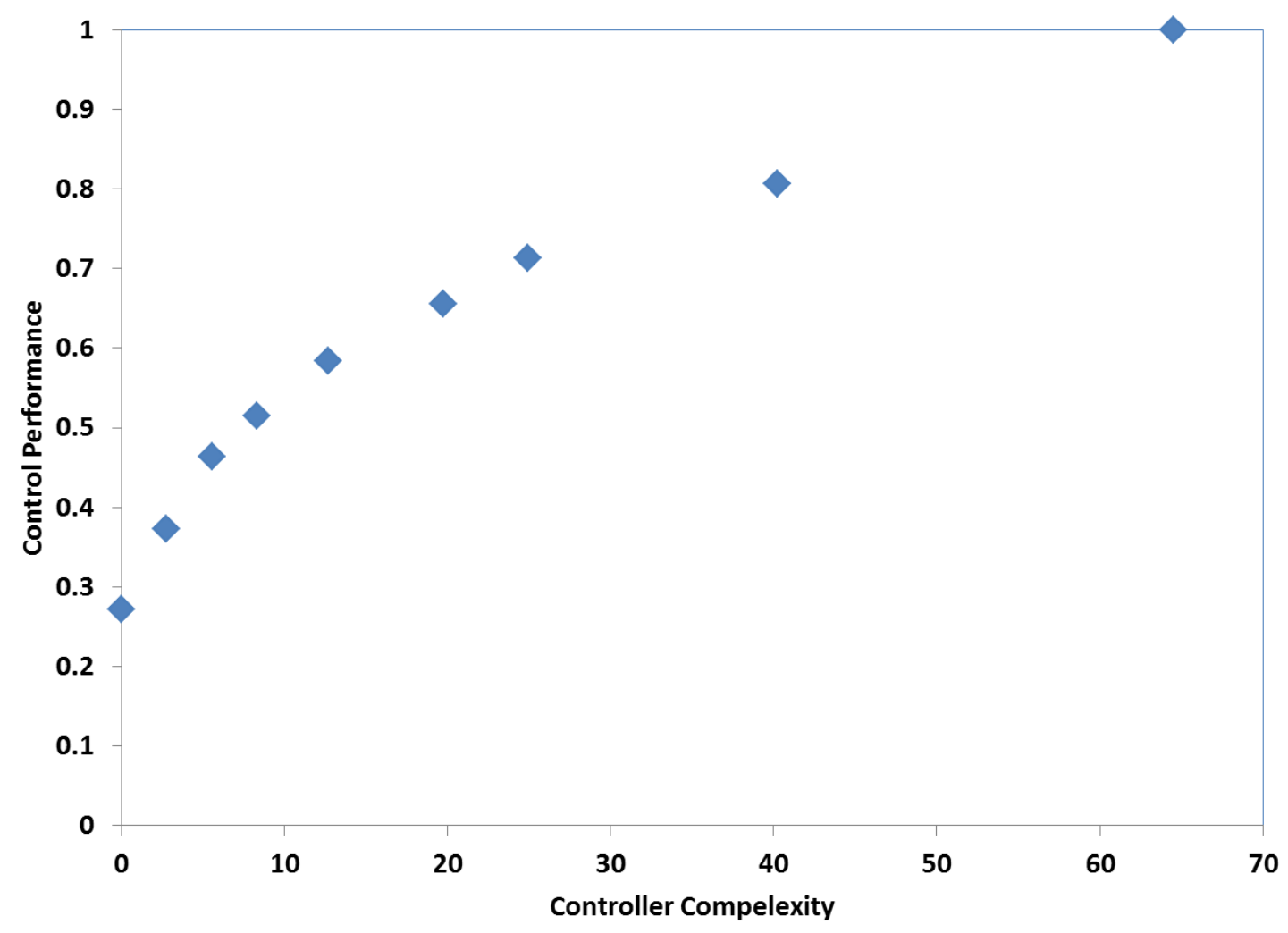

Figure 13. Pareto Set for Control Structure using PM Interaction Measure

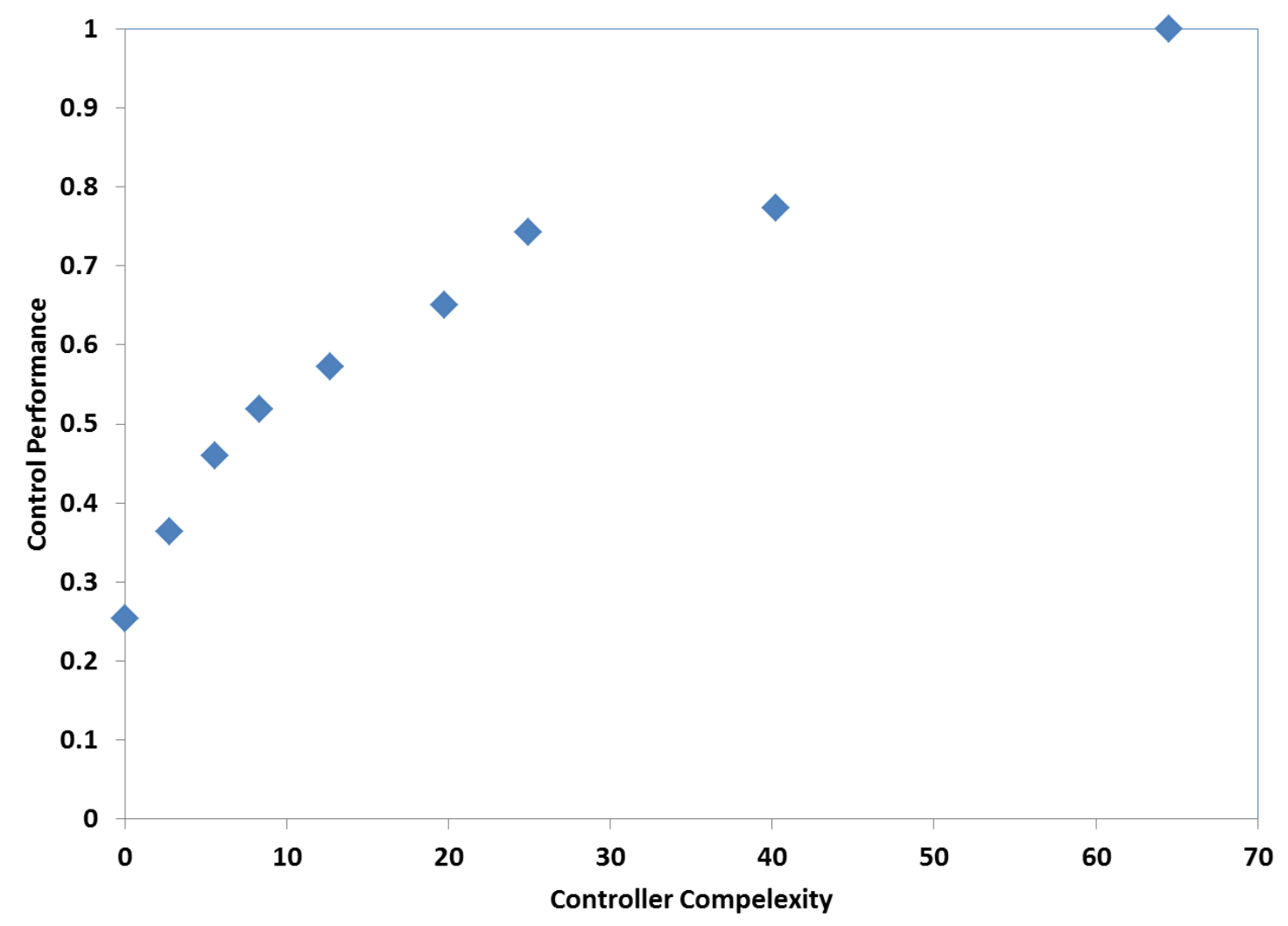

Figure 14. Pareto Set for Control Structure using $\Sigma_{2}$ Interaction Measure 
Table 23. Numerical Derivative of Control Performance with respect to Controller Complexity [listed in increasing controller complexity]

\begin{tabular}{|c|c|c|c|}
\hline Controller Form & Sig & PM & HIIA \\
\hline Decentralized & - & - & - \\
\hline One 2 by 2 Centralized & 0.039638 & 0.036428 & 0.040864 \\
\hline Two 2 by 2 Centralized & 0.034841 & 0.033038 & 0.030405 \\
\hline Three 2 by 2 Centralized & 0.021244 & 0.018322 & 0.012263 \\
\hline $\begin{array}{c}\text { One } 2 \text { by 2 Centralized } \\
\text { One } 3 \text { by } 3 \text { Centralized }\end{array}$ & 0.012298 & 0.015821 & 0.014232 \\
\hline One 4 by 4 Centralized & 0.010963 & 0.010176 & 0.009009 \\
\hline Two 3 by 3 Centralized & 0.01767 & 0.011027 & 0.006856 \\
\hline $\begin{array}{c}\text { One } 4 \text { by 4 Centralized } \\
\text { One } 2 \text { by 2 Centralized }\end{array}$ & 0.002028 & 0.00617 & 0.007597 \\
\hline One 6 by 6 Centralized & 0.009358 & 0.007953 & 0.006746 \\
\hline
\end{tabular}

\subsubsection{Supervisory Controller Design}

With the structure of the supervisory control determined, the design of the control system is next completed. As mentioned in Section 4.2.2.2, the optimal structure that balances control performance and controller complexity are two centralized controllers. One $4 \times 4$ centralized control of $\mathrm{CO}_{2}$ capture, vapor composition in the $\mathrm{CO}_{2}$ absorber, $\mathrm{H}_{2} \mathrm{~S}$ purity to the Claus unit, and solvent composition in the $\mathrm{H}_{2} \mathrm{~S}$ absorber using the LP flash pressure, semi-lean solvent flowrate, lean solvent flowrate, and $\mathrm{H}_{2} \mathrm{~S}$ concentrator pressure. The second centralized controller controls $\mathrm{H}_{2} \mathrm{~S}$ capture and water content of the solvent using the stripper bottom temperature and steam flow to the stripper. For the purposes of this work, the forms used for these centralized controls are linear model predictive controls (LMPC).

Models were identified by applying a pseudorandom binary sequence (PRBS) input signal to the nonlinear process model in Aspen Plus Dynamics. The PRBS input signal used for the semilean solvent flow rates can be seen in Figure 15. The corresponding output data for $\mathrm{CO}_{2}$ capture, $\mathrm{CO}_{2}$ vapor fraction in $\mathrm{CO}_{2}$ absorber, $\mathrm{CO}_{2}$ liquid fraction in $\mathrm{H}_{2} \mathrm{~S}$ absorber, $\mathrm{H}_{2} \mathrm{~S}$ capture, water composition of the solvent, and the purity of the off-gas sent to the Claus unit from the nonlinear process model as well as the predictions from the linear, identified transfer functions are shown as Figures 16, 17, 18, 19, 20, and 21, respectively. Using the MATLAB system identification toolbox, the output data and the PRBS input data were used to identify linear transfer functions. Using these identified models, the LMPCs for the process are designed. 


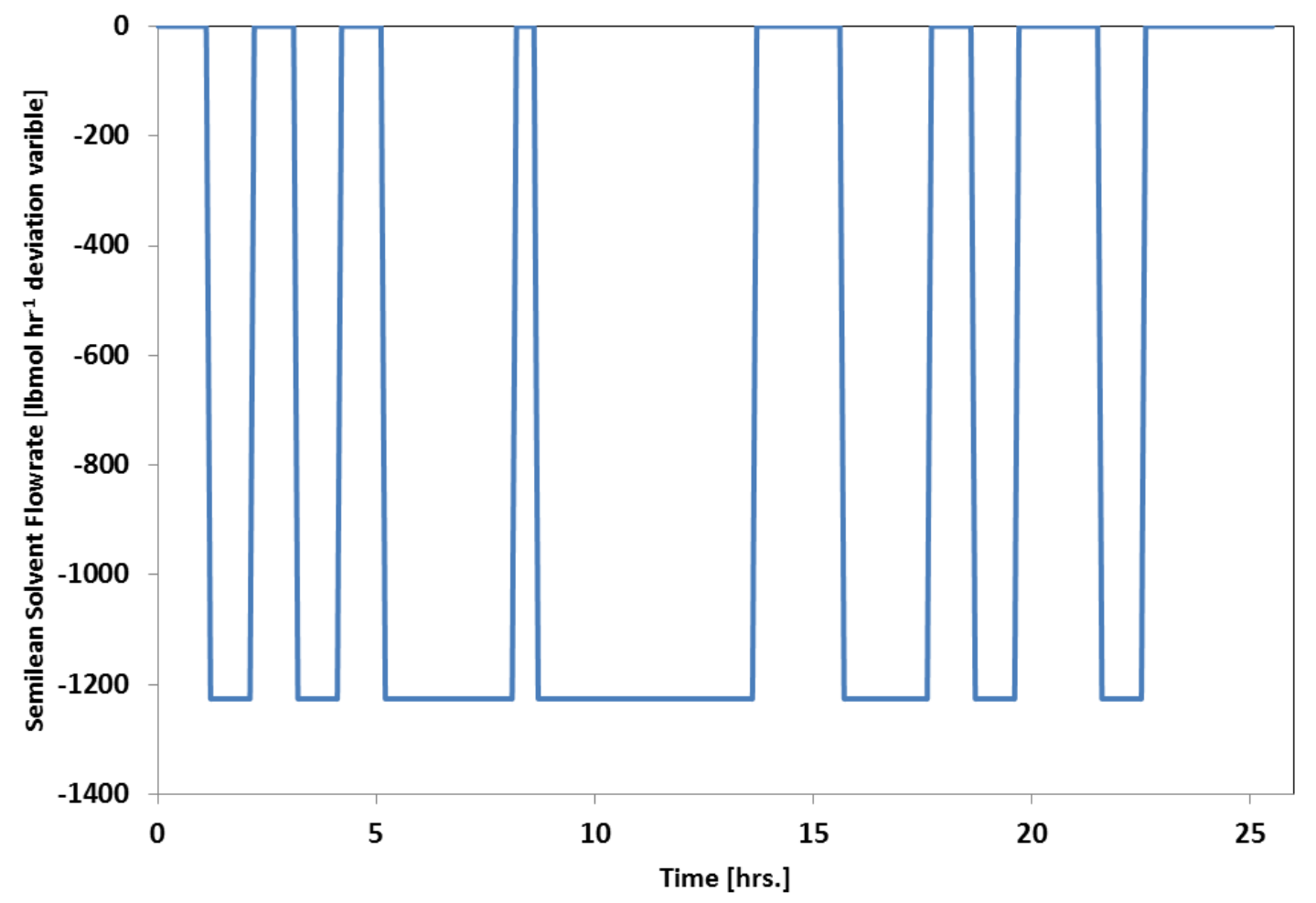

Figure 15. PRBS Input used for Semi-lean Solvent Flow

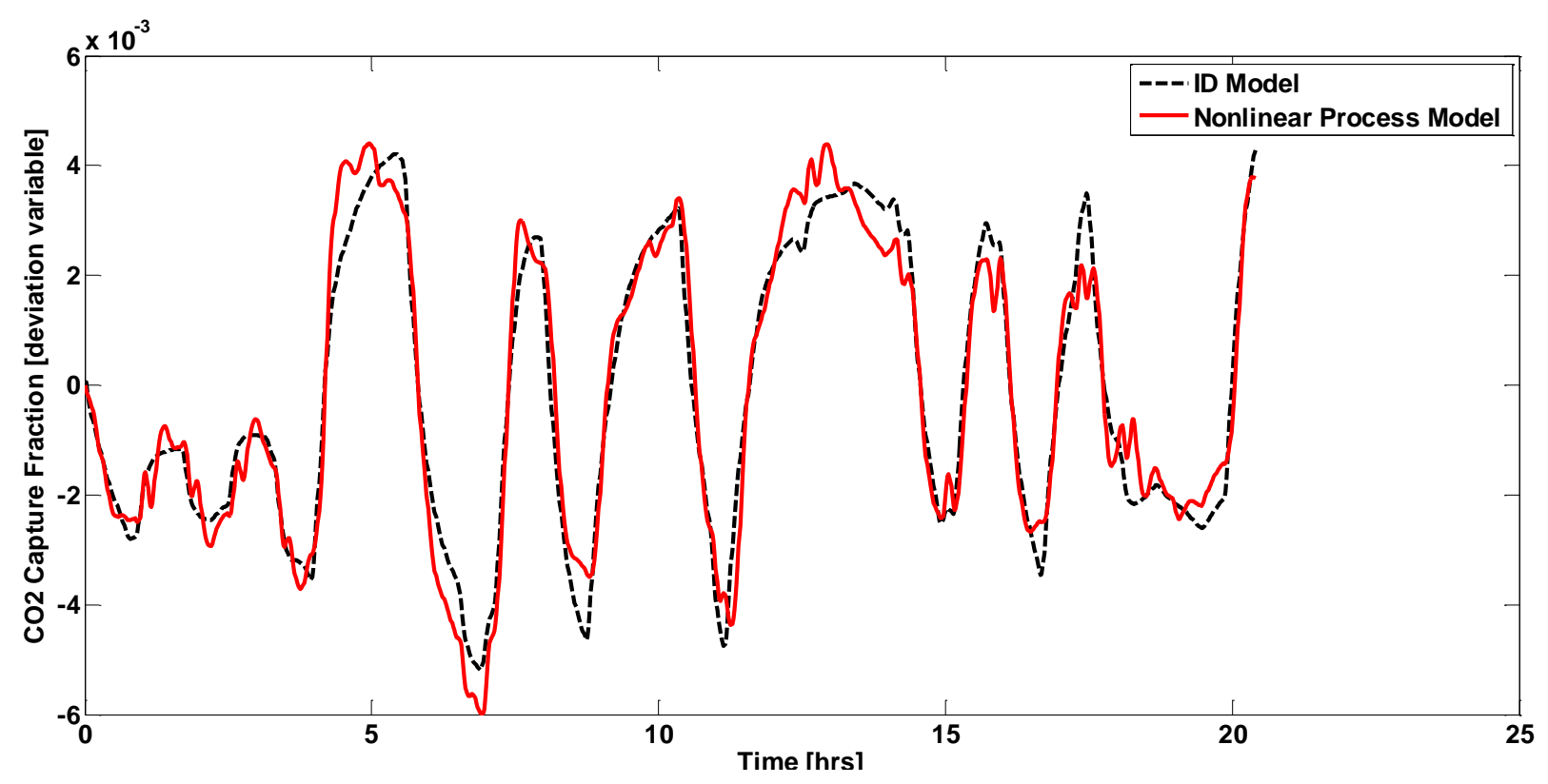

Figure 16. Effect of PRBS input signal on $\mathrm{CO}_{2}$ Capture and Comparison with Identified Model 


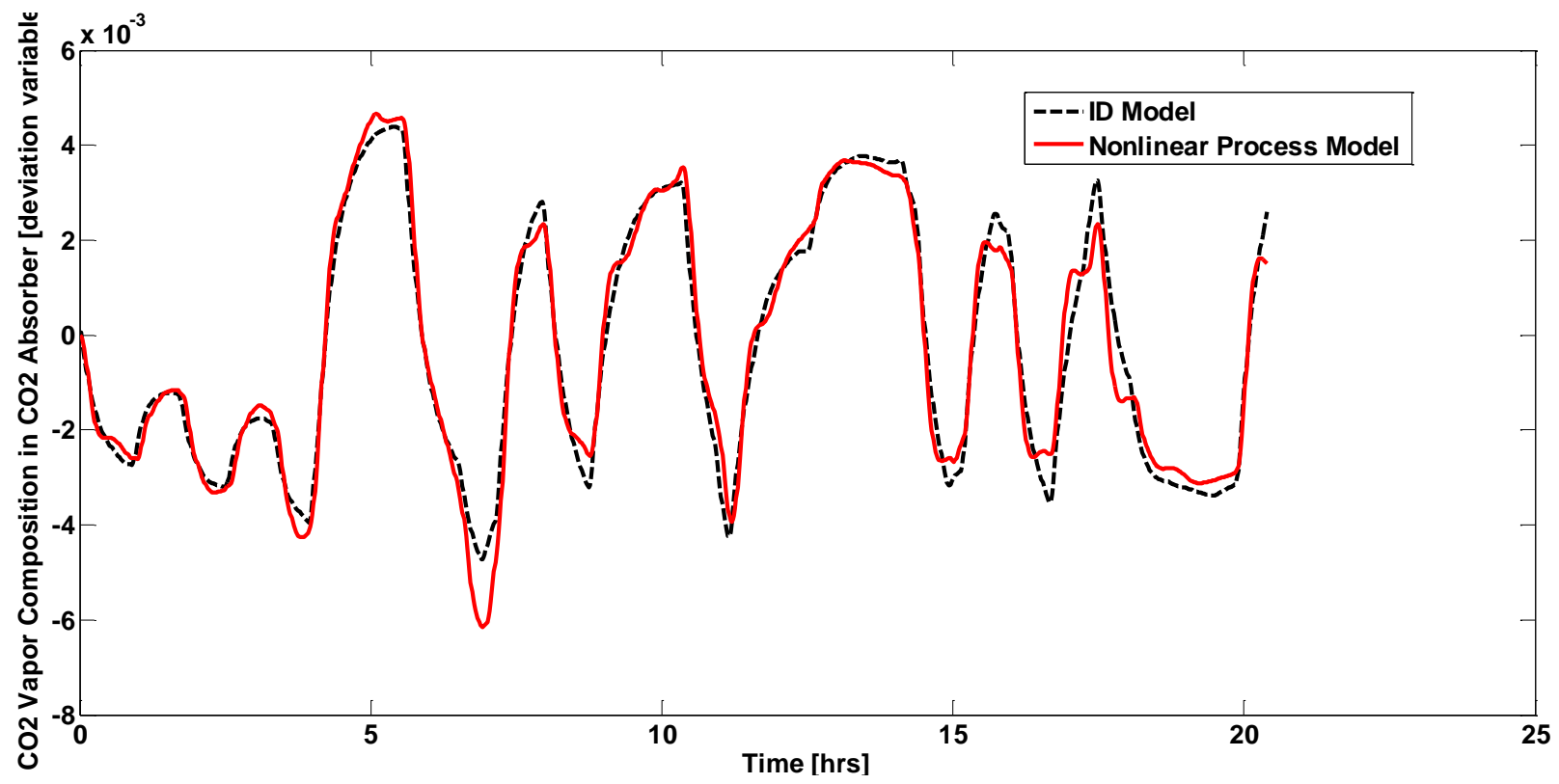

Figure 17. Effect of PRBS input signal on $\mathrm{CO}_{2}$ Vapor Composition in $\mathrm{CO}_{2} \mathrm{Absorber}$ and Comparison with Identified Model

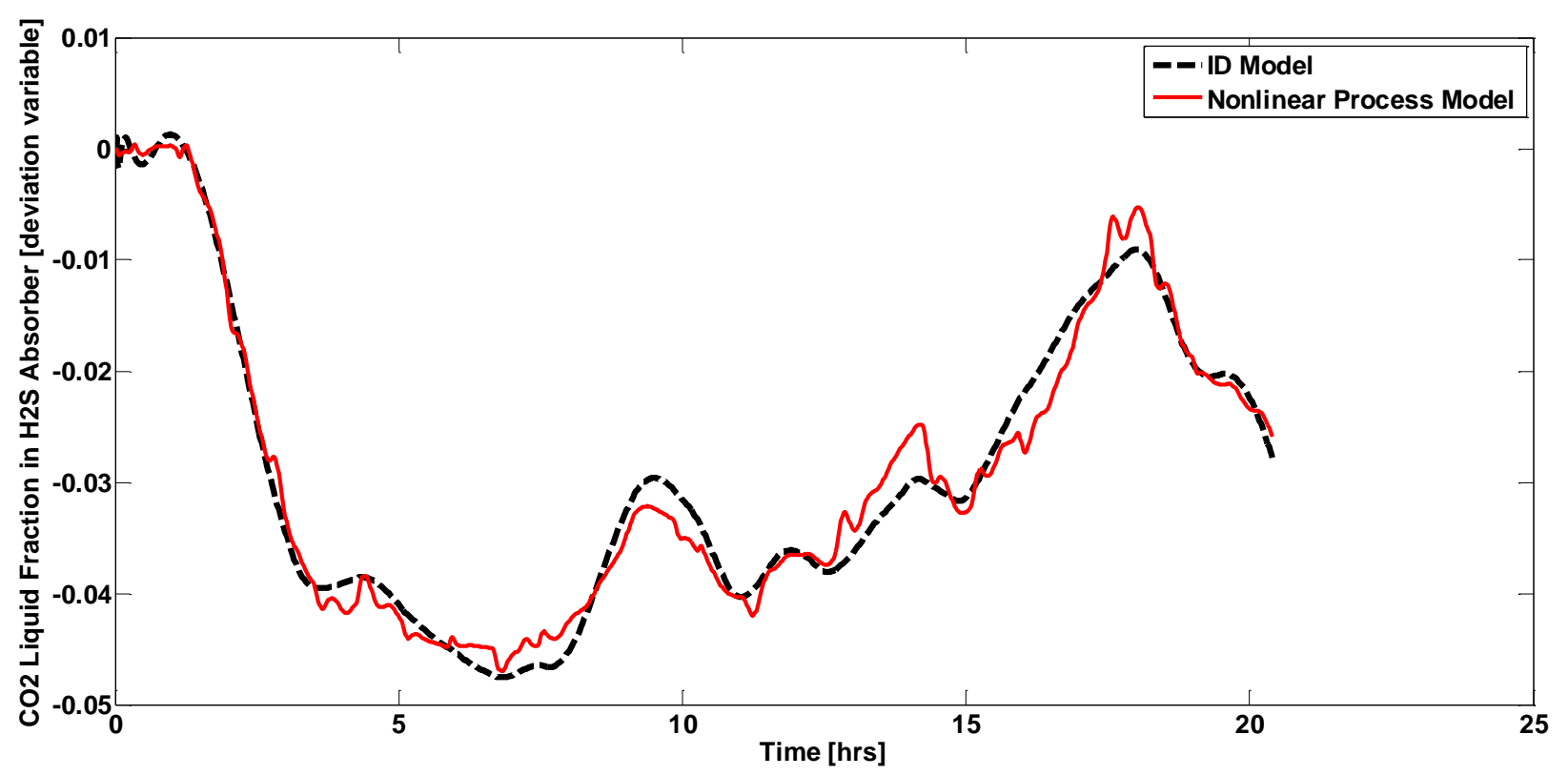

Figure 18. Effect of PRBS input signal on Solvent Composition in $\mathrm{H}_{2} \mathrm{~S}$ Absorber and Comparison with Identified Model 


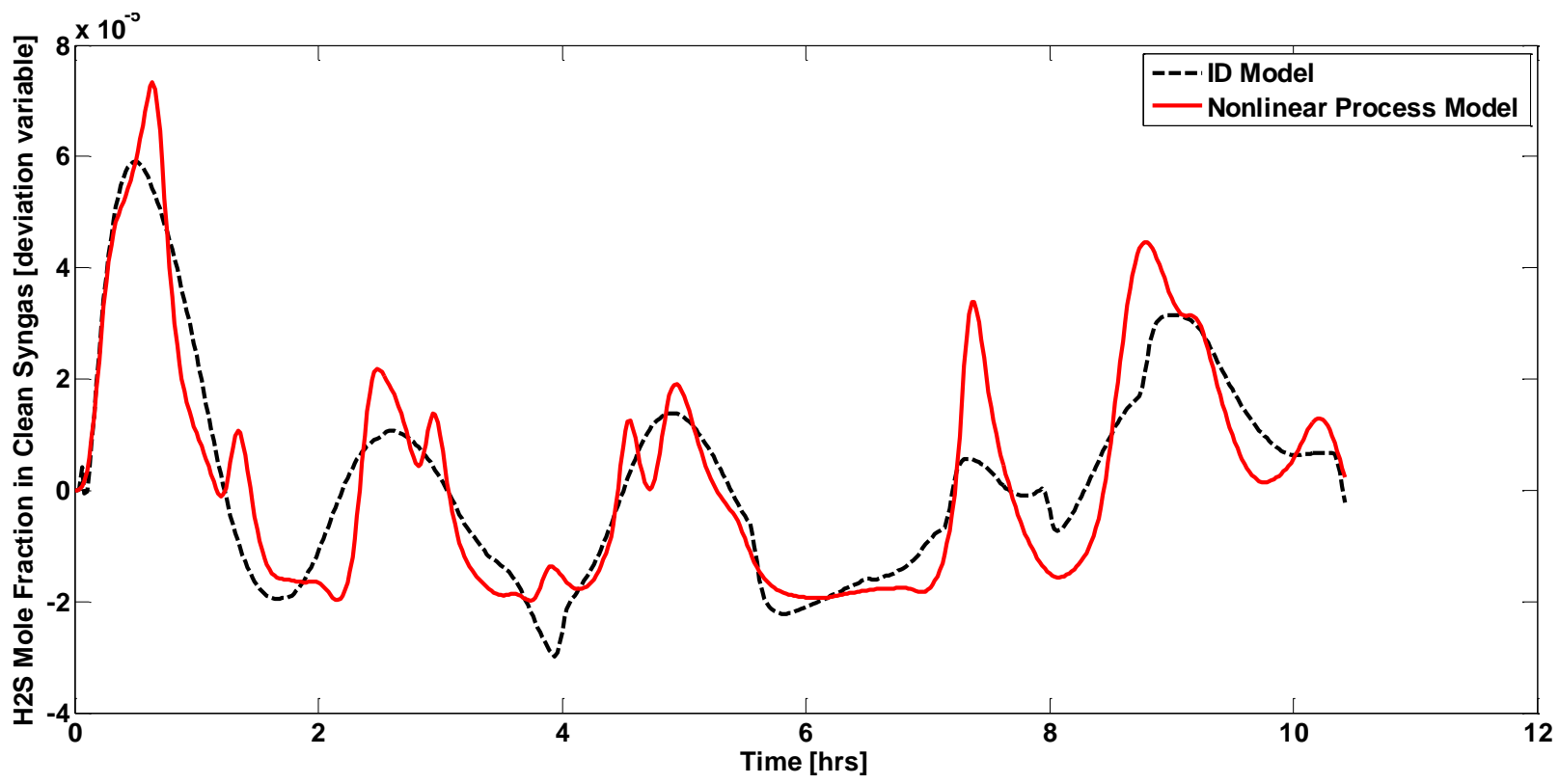

Figure 19. Effect of PRBS input signal on $\mathrm{H}_{2} \mathrm{~S}$ Capture and Comparison with Identified Model

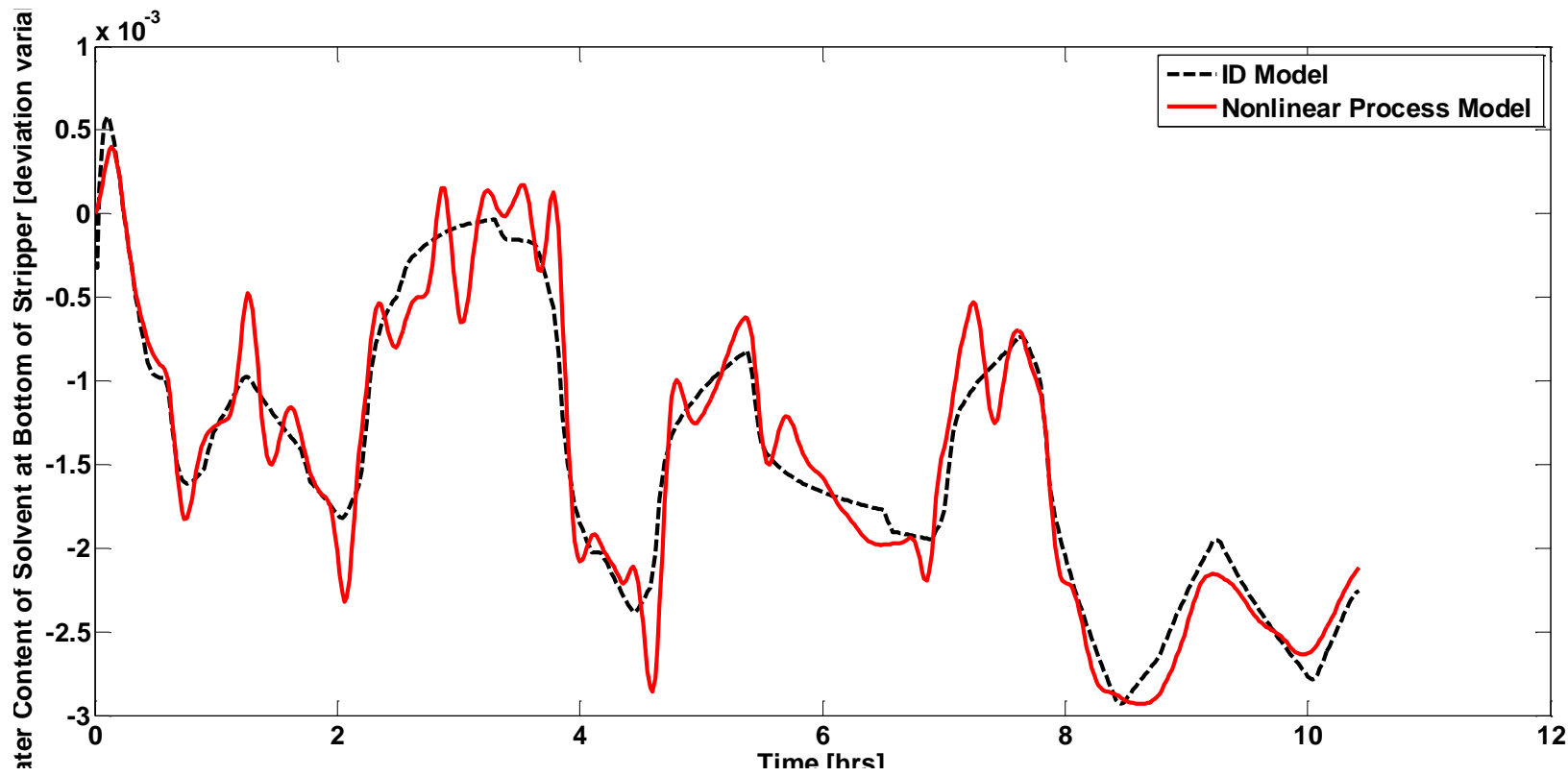

Figure 20. Effect of PRBS input signal on Water Composition of Solvent and Comparison with Identified Model 


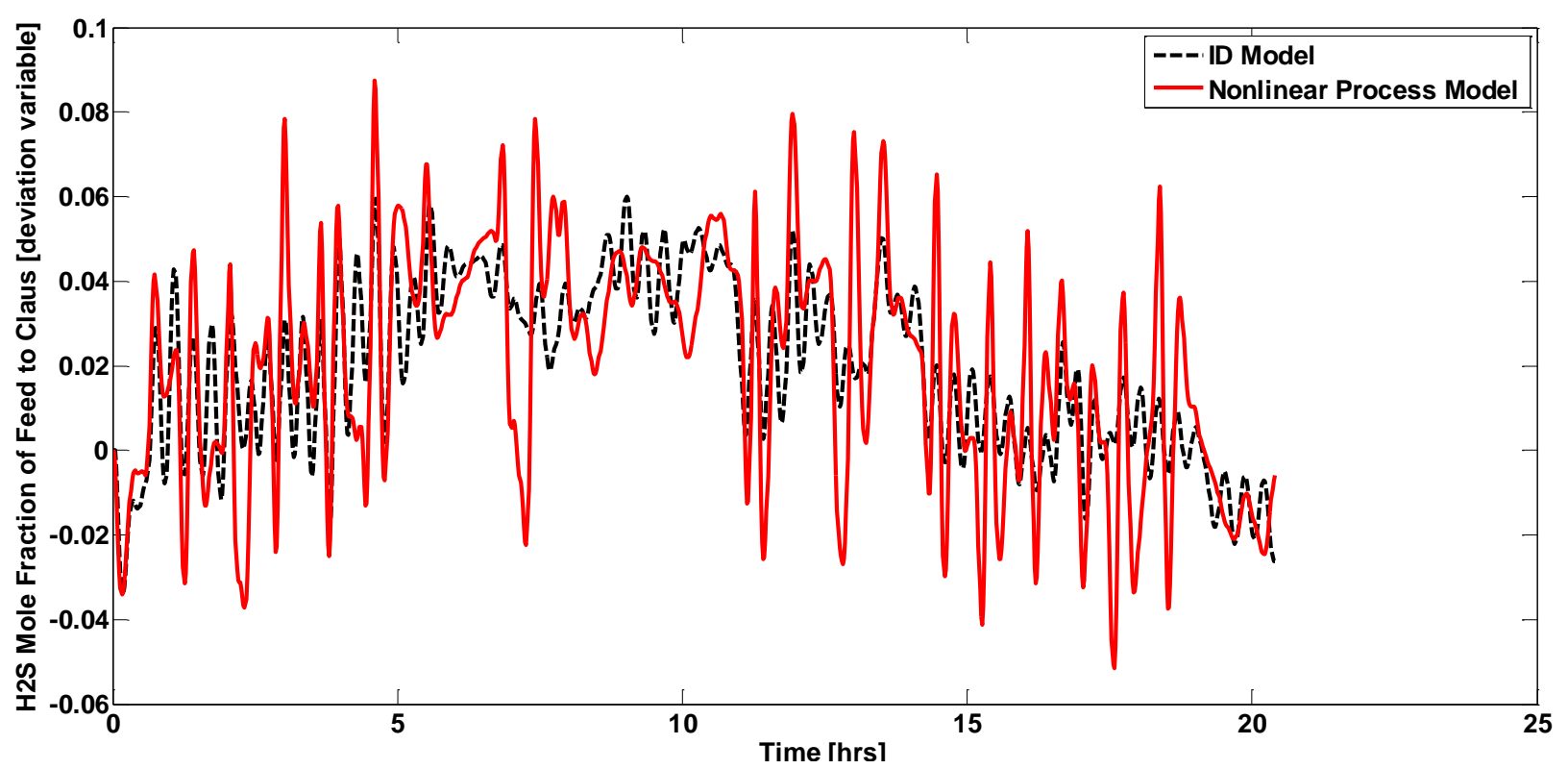

Figure 21. Effect of PRBS input signal on $\mathrm{H}_{2} \mathrm{~S}$ Purity to Claus Unit and Comparison with Identified Model

For the tuning of the LMPCs, the economic information obtained during primary controlled variable selection is introduced. From the analysis completed during primary controlled variable selection, it is known how a particular controlled variable affects the economics of the process. For example, if it is known that the absolute deviation of variable $A$ affects the economics twice as much as the absolute deviation of variable $B$, it could be said that the control of variable $A$ is twice as important. It is from this perspective that the tuning of the LMPCs is undertaken. The objective of the optimization is the minimization of Equation 53. Here, $n_{u}$ is the number of primary controlled variables, ISE is the integral squared error of the primary controlled variable $i$, and $\Theta_{i}$ is the scaling factor based upon the economics of the process. Table 24 shows comparison of the objective function values of the initial, non-optimized tuning used for the two LMPCs and that of the PID controls. Using these tuning parameters, superior performance, as compared to PID control, is attained. From this initial case, an optimization is carried out to minimize Equation 53. Equation 53 is linear representation of the economic losses incurred due to errors in the primary controlled variables. The assumption made in the formulation of Equation 53 is that self-optimizing controlled variables are 'perfect' self-optimizing controlled variables, i.e., their current setpoint is the optimal setpoint, and that the economics of the process are linear functions of the errors in the controlled variable loops. Therefore, the result of Equation 53 is economic loss due to error in controlled variables. The optimization problem to 
be solved for this case is shown as Equation 54. This optimization is undertaken using a quasiNewton method. Five iterations were carried out to solve Equation 54 considering a -20\% step change in syngas flow as the disturbance. This optimization improved the performance of the LMPC from \$222.7 to \$177.1, a 20\% improvement from the base case. These values are based upon the entire time range of operation till the process settles. The time series result is shown in Figure 22. Figure 23, 24, and 25 show the comparison of the dynamic responses of the LMPC controls with that of the PID controls for a $-20 \%$ step change in syngas flow for $\mathrm{CO}_{2}$ capture, $\mathrm{H}_{2} \mathrm{~S}$ purity to Claus unit, and $\mathrm{CO}_{2}$ vapor fraction in $\mathrm{CO}_{2}$ absorber, respectively. Table 25 shows the corresponding ISE values of the LMPC control and that of the PID control. It is clear from Table 25 that the disturbance rejection characteristics for some primary controlled variables are improved while others are made worse. This is to be expected as the goal of the optimization carried out for tuning of the LMPC was to improve the economics of the process. Certain variables, like water composition of the solvent, do not have a large impact on the economics of the process, so its performance was sacrificed for the improvement of variables that have a larger importance on the economics, e.g., fraction $\mathrm{CO}_{2}$ in the vapor of the $\mathrm{CO}_{2}$ absorber.

$$
\begin{gathered}
\sum_{i=0}^{n_{u}} \Theta_{i} I S E_{y_{i}} \\
\min _{Q, R} \sum_{i=0}^{n_{u}} \Theta_{i} I S E_{y_{i}} \\
\min _{u}\left(\sum_{p=1}^{T} Q\left(r_{p}-y_{p}\right)^{2}+\sum_{p=1}^{T} R \Delta u_{p}^{2}\right)
\end{gathered}
$$

Table 24. Comparison of Initial LMPC to PID for Three Disturbances

\begin{tabular}{|c|c|c|c|}
\hline Disturbance & PID & LMPC & Percent Improvement \\
\hline$-20 \%$ Step in syngas flow & 701.4 & 222.7 & $68.25 \%$ \\
\hline$+2 \%$ Step in $\mathrm{CO}_{2}$ Capture & 116.9 & 43.0 & $63.20 \%$ \\
\hline$-2 \%$ Step in $\mathrm{CO}_{2}$ Capture & 103.7 & 49.3 & $52.43 \%$ \\
\hline
\end{tabular}




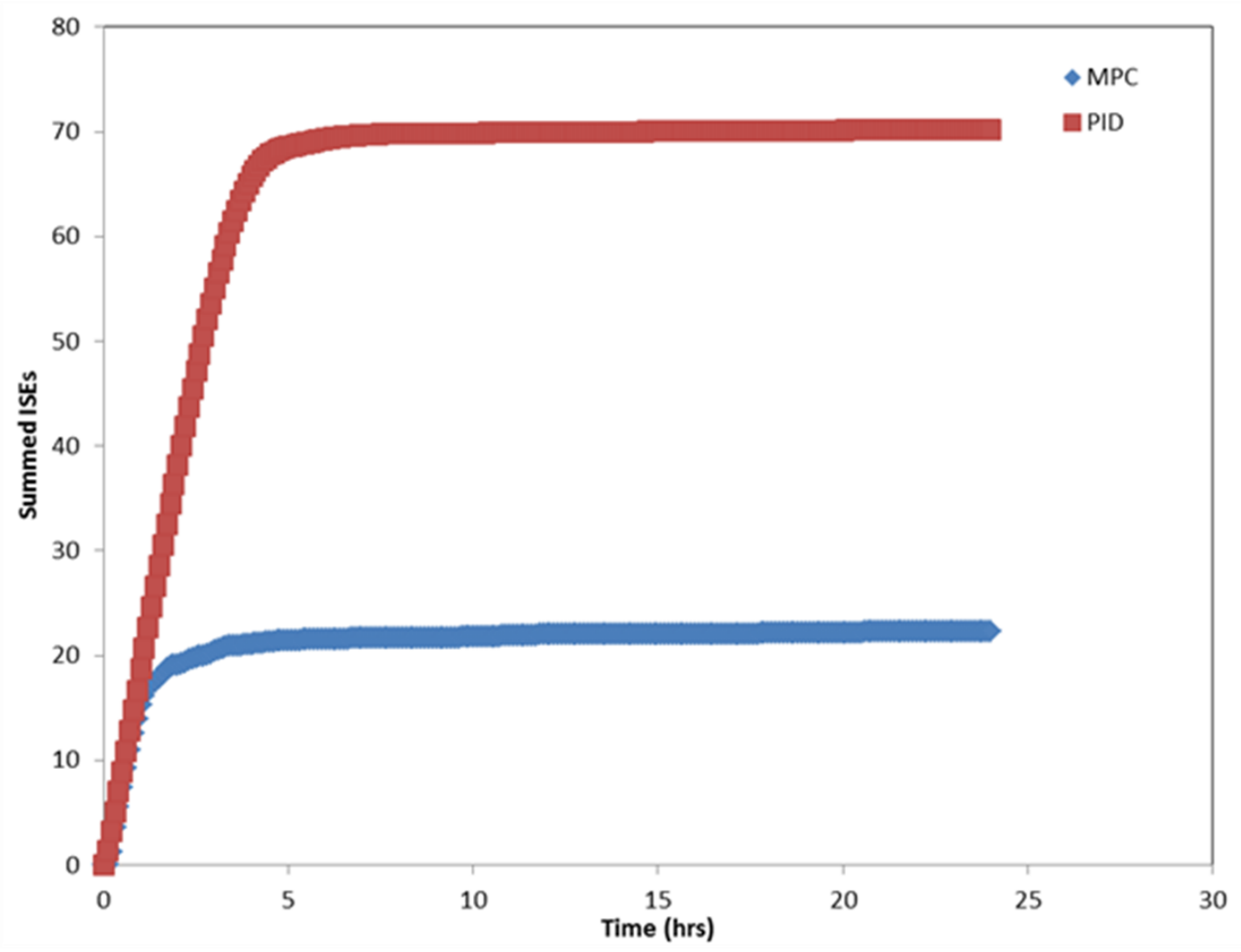

Figure 22. Economic Loss as a Function of Time due to -20\% Step in Syngas Flow

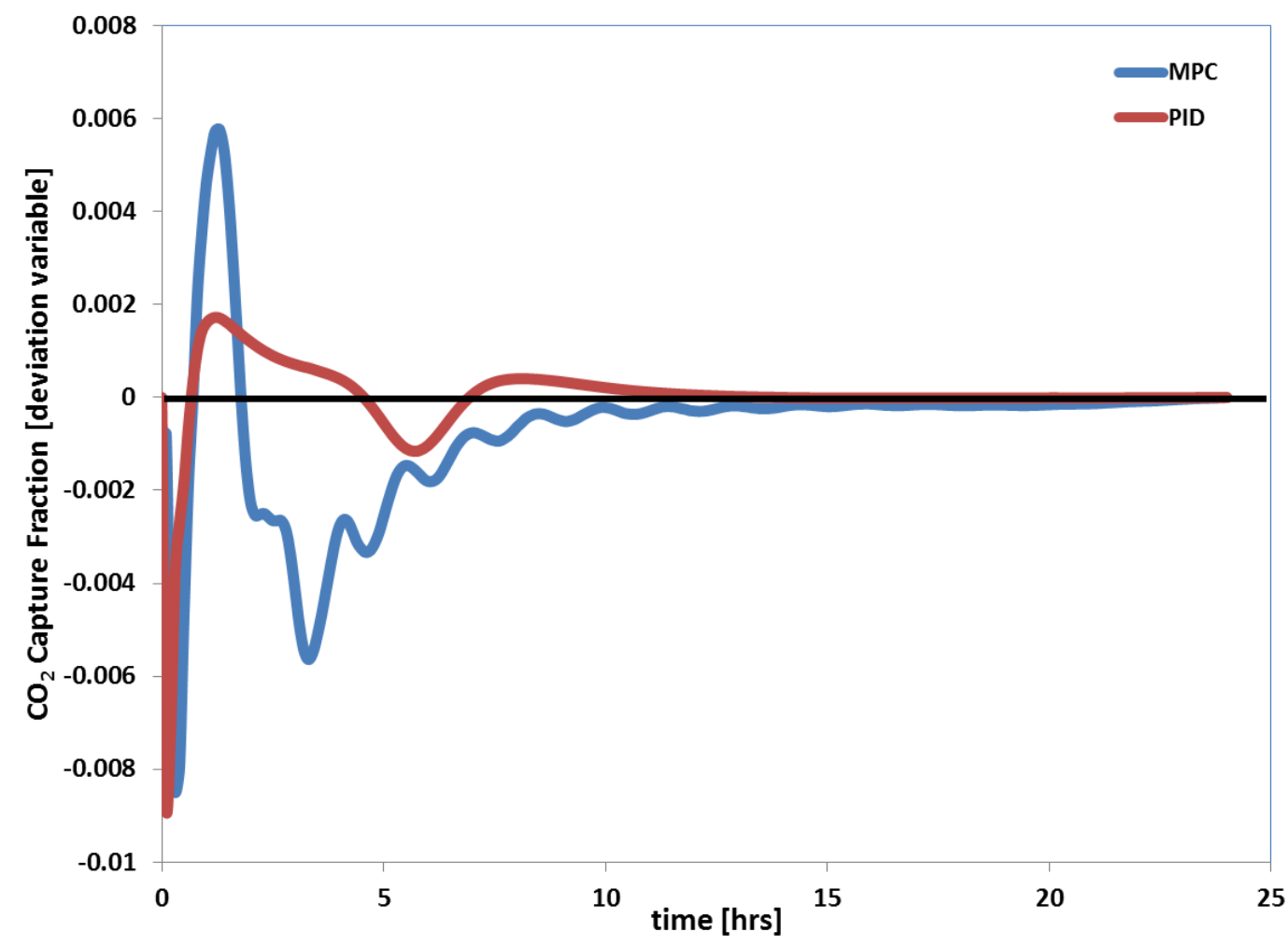

Figure 23. $\mathrm{CO}_{2}$ Capture Fraction after -20\% Step Change in Syngas Flow 


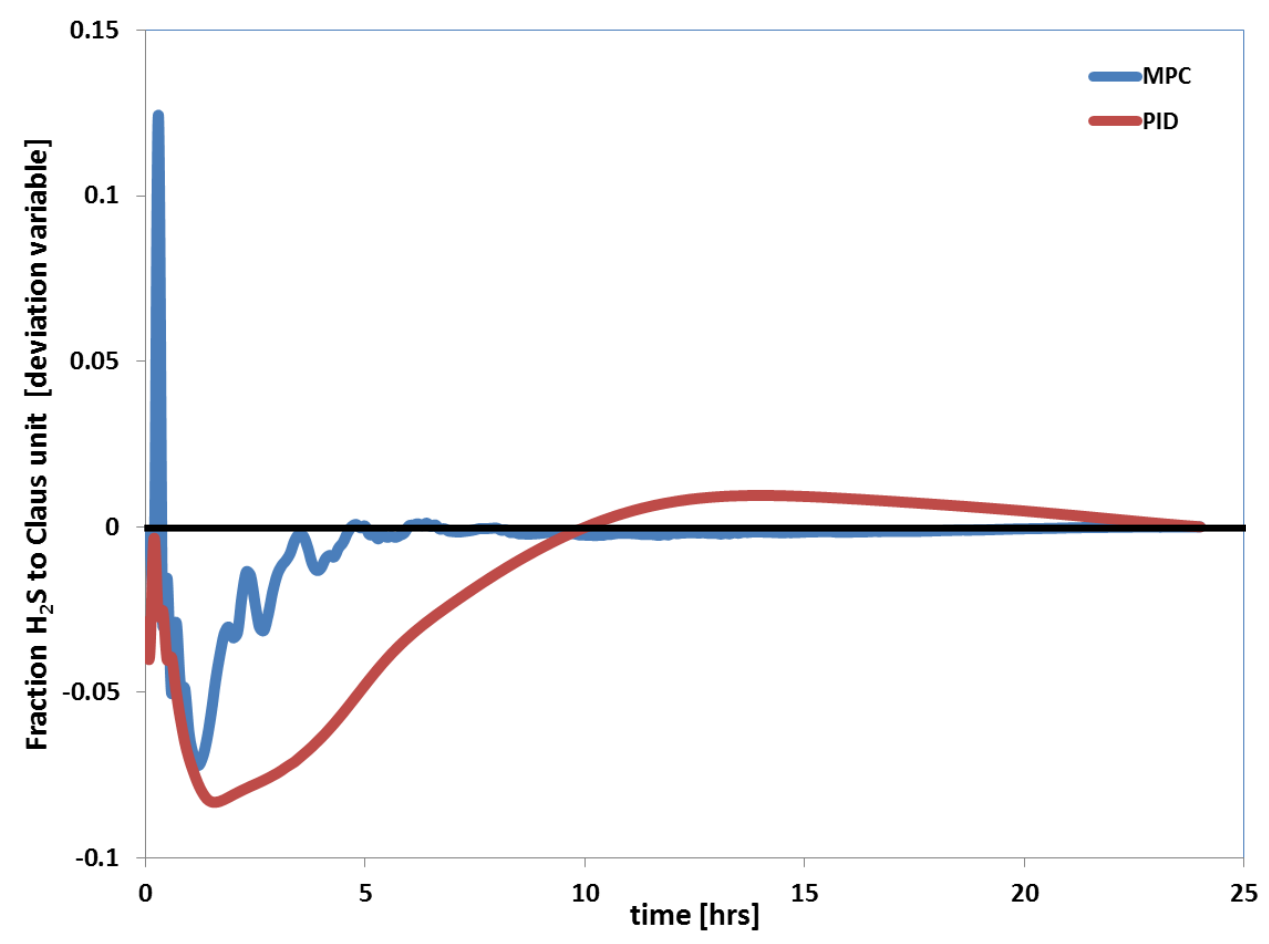

Figure 24. $\mathrm{H}_{2} \mathrm{~S}$ Purity to Claus after $-20 \%$ Step Change in Syngas Flow

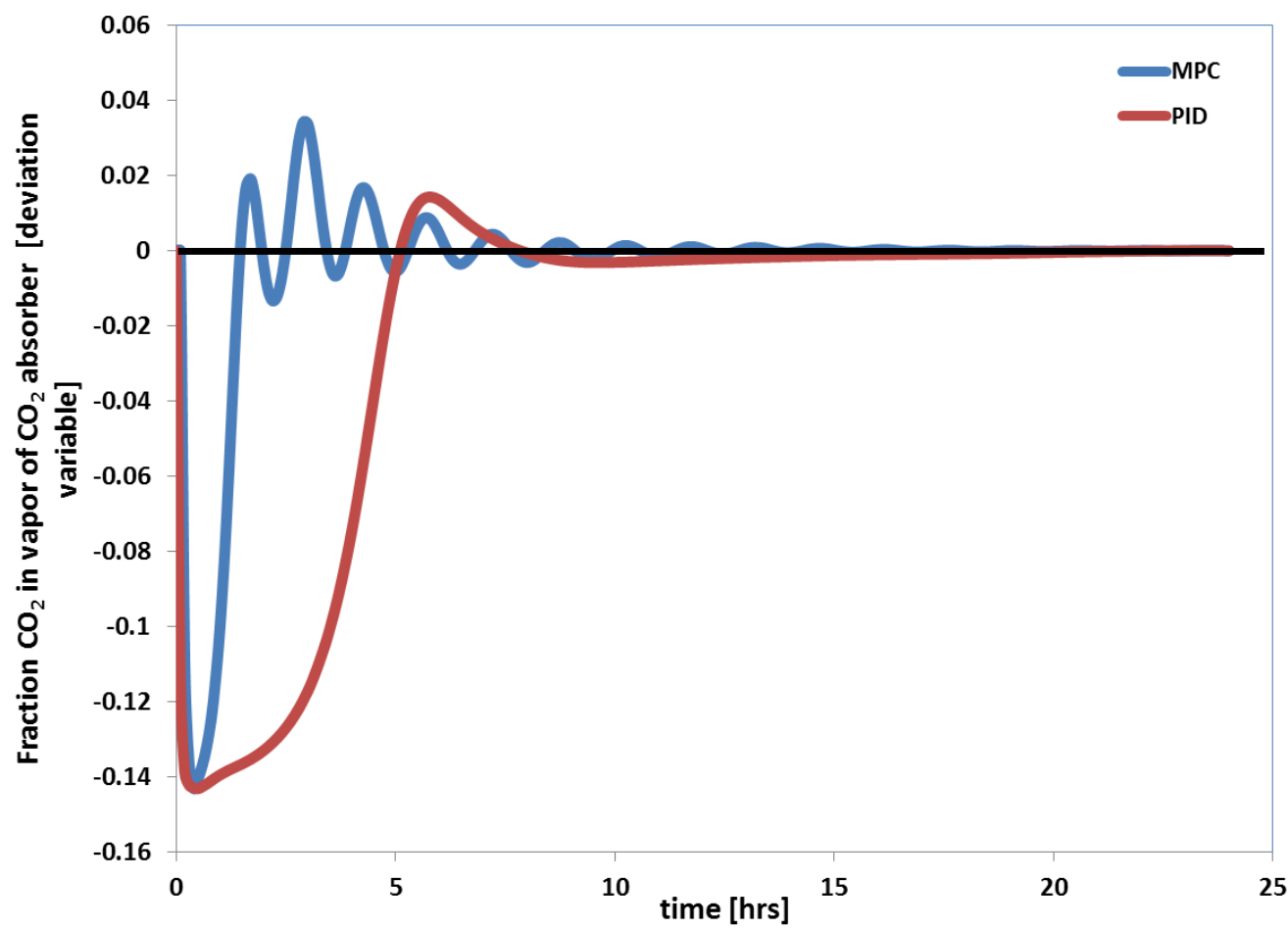

Figure 25. $\mathrm{CO}_{2}$ Vapor Fraction in $\mathrm{CO}_{2}$ Absorber after -20\% Step Change in Syngas Flow 
Table 25. Comparison of ISE Values for a -20\% Step Change in Syngas Flow

\begin{tabular}{|l|c|c|c|}
\hline & PID & MPC & $\begin{array}{c}\text { Percent } \\
\text { Improvement }\end{array}$ \\
\hline $\mathrm{CO}_{2}$ Capture & 0.089128 & 0.267443 & -200 \\
\hline $\mathrm{H}_{2} \mathrm{~S}$ Capture & 0.000892 & 0.000649 & 27 \\
\hline Water Content of Solvent & 0.018939 & 0.054999 & -190 \\
\hline $\mathrm{H}_{2} \mathrm{~S}$ Purity to Claus & 7.379743 & 1.401381 & 81 \\
\hline $\begin{array}{l}\mathrm{CO}_{2} \text { Vapor Fraction in } \mathrm{CO}_{2} \\
\text { Absorber }\end{array}$ & 5.743467 & 1.929164 & 66 \\
\hline $\begin{array}{l}\mathrm{CO}_{2} \text { Liquid Fraction in } \mathrm{H}_{2} \mathrm{~S} \\
\text { Absorber }\end{array}$ & 0.638438 & 1.704903 & -167 \\
\hline
\end{tabular}

By applying this procedure, an optimal control system has been designed for the AGR unit of an IGCC power plant with $\mathrm{CO}_{2}$ capture. The steady state economics of the process have been improved by $27 \%$ from the base case. The primary controlled variables identified reduce economic loss due to off-design operations by as much as $94 \%$ from the case were only active constraints are controlled. Secondary controlled variables were identified that improve the control performance of the primary control loops by reducing loop dead-times and undesired dynamics. Finally, a supervisory controller consisting of two LMPCs have been designed to optimally control the process with minimal control complexity.

\subsection{Gasification Section of IGCC}

In this section, the application of the design procedure to the gasification section of the IGCC power plant will be discussed. It should be noted that the gasification section is less complex than the AGR unit, due primarily to the number of manipulated variables.

\subsubsection{Optimization}

As with the AGR unit, the design procedure begins by optimizing the process at nominal and offdesign conditions. For this optimization, the AGR unit, along with its control structure, is included. In this way, the effects of the gasification section on the AGR unit will be accounted for within the optimization. The objective function used for the optimization of the gasification section is given as Equation 53. Like the objective function used for the AGR unit, this objective function represents the economic cost of operations $\left(\$ \mathrm{~h}^{-1}\right)$. The value of the syngas or generated electricity is not considered within this optimization as the production rate is considered as a constraint during optimization. This optimization reduced the operational cost of the gasification 
section by $12 \%$. In addition to nominal operation, this optimization was carried out at various production rates, carbon capture percentage, and variations in coal type. Table 26 shows the offdesign conditions investigated.

$$
J\left[\frac{\$}{\mathrm{hr}}\right]=47.52\left(\dot{m}_{\text {coal }}\right)\left[\frac{\text { short ton }}{h r}\right]+0.04161\left(\dot{n}_{\text {oxygen }}\right)\left[\frac{\mathrm{kmol}}{\mathrm{hr}}\right]+0.03957\left(\dot{m}_{\text {steam }}\right)+94.3\left(W_{\text {slurrypump }}\right)+J_{A G R}
$$

Table 26. Disturbances Considered

\begin{tabular}{|l|c|c|c|}
\hline Disturbance & Nominal & Low & High \\
\hline Carbon Capture & $90 \%$ & $88 \%$ & $92 \%$ \\
\hline Syngas Flow Rate & $100 \%$ & $80 \%$ & $100 \%$ \\
\hline
\end{tabular}

These optimizations identified 3 active constraints: water-to-coal ratio, gasifier outlet temperature, and syngas production rate. The manipulated variables used for the control of these active constraints are shown in Table 27. After the control of the active constraints is complete, there exists only one unpaired manipulated variable, the shift steam flowrate.

Table 27. Active Constraints and Pairings

\begin{tabular}{|l|l|}
\hline \multicolumn{1}{|c|}{ Active Constraint } & \multicolumn{1}{c|}{ Manipulated Variable } \\
\hline Carbon Capture & Low Pressure Flash Pressure \\
\hline Water to Coal Ratio & Slurry Water Flowrate \\
\hline Gasifier Outlet Temperature & Oxygen Flowrate \\
\hline Syngas Flowrate & Coal Flowrate \\
\hline
\end{tabular}

\subsubsection{Primary Controlled Variable Selection}

Unlike the AGR unit, there are very few possible controlled variable sets for the gasification section of the IGCC power plant. For this reason, enumeration of all possible controlled variable sets was undertaken for this process. Table 28 shows all the candidate controlled variables considered for the shift steam flowrate. After enumeration of all these possibilities, only the carbon monoxide conversion was found to be a member of the Pareto set. This makes intuitive sense as the gain of the steam flow on the other candidate variables, e.g., temperatures, is not as large as it is on carbon monoxide conversion. Additionally, carbon monoxide conversion is a 
rather intuitive primary controlled variable for the process as the primary function of the water gas shift reactors and the shift steam is conversion of carbon monoxide.

Table 28. List of Candidate Controlled Variables

\begin{tabular}{|l|}
\hline $\mathrm{CO}$ conversion in water gas shift reactors \\
\hline $\mathrm{CO}$ fraction of gas entering $1^{\text {st }}$ water gas shift reactor \\
\hline $\mathrm{CO}$ fraction of gas entering $2^{\text {nd }}$ water gas shift reactor \\
\hline $\mathrm{CO}$ fraction of gas exiting $2^{\text {nd }}$ water gas shift reactor \\
\hline Temperature of gas entering $1^{\text {st }}$ water gas shift reactor \\
\hline Temperature of gas entering $2^{\text {nd }}$ water gas shift reactor \\
\hline Temperature of gas exiting $2^{\text {nd }}$ water gas shift reactor \\
\hline $\mathrm{CO}_{2}$ fraction of gas entering $1^{\text {st }}$ water gas shift reactor \\
\hline $\mathrm{CO}_{2}$ fraction of gas entering $2^{\text {nd }}$ water gas shift reactor \\
\hline $\mathrm{CO}_{2}$ fraction of gas exiting $2^{\text {nd }}$ water gas shift reactor \\
\hline $\mathrm{H}_{2} \mathrm{O}$ fraction of gas entering $1^{\text {st }}$ water gas shift reactor \\
\hline $\mathrm{H}_{2} \mathrm{O}$ fraction of gas entering $2^{\text {nd }}$ water gas shift reactor \\
\hline $\mathrm{H}_{2} \mathrm{O}$ fraction of gas exiting $2^{\text {nd }}$ water gas shift reactor \\
\hline
\end{tabular}

\subsubsection{Secondary Controlled Variable Selection}

For the selection of secondary controlled variables for the gasification section, it is assumed that the control structure of the process is that of a gas turbine lead, gasifier follow configuration. This configuration uses the coal feed to the gasifier to control the system pressure. The other configuration possible is the gasifier lead, gas turbine follow. In this configuration, syngas flow to the gas turbine is used to control system pressure. Additionally, it is assumed that the gasifier outlet temperature is controlled by the oxygen flowrate to the gasifier via the secondary controlled variable of the oxygen-to-coal ratio.

Secondary controlled variables are selected for the primary controlled variables of carbon capture and carbon monoxide conversion. The candidate secondary controlled variables considered are listed in Table 29. It is important to note that during the control system design for the AGR unit that $\mathrm{CO}_{2}$ capture is a constraint. However, in reality the true constraint for the IGCC power plant is carbon capture, not $\mathrm{CO}_{2}$ capture. Therefore, $\mathrm{CO}_{2}$ capture is a candidate secondary controlled variable for the process. Previously, it was assumed that the $\mathrm{CO}_{2}$ capture 
would be selected as a secondary controlled variable. Here it is determined whether or not this assumption is correct.

Table 29. List of Candidate Secondary Controlled Variables

\begin{tabular}{|l|}
\hline $\mathrm{CO}_{2}$ capture in AGR \\
\hline $\mathrm{CO}$ fraction of gas entering $1^{\text {st }}$ water gas shift reactor \\
\hline $\mathrm{CO}$ fraction of gas entering $2^{\text {nd }}$ water gas shift reactor \\
\hline $\mathrm{CO}$ fraction of gas exiting $2^{\text {nd }}$ water gas shift reactor \\
\hline Temperature of gas entering $1^{\text {st }}$ water gas shift reactor \\
\hline Temperature of gas entering $2^{\text {nd }}$ water gas shift reactor \\
\hline Temperature of gas exiting $2^{\text {nd }}$ water gas shift reactor \\
\hline $\mathrm{CO}_{2}$ fraction of gas entering $1^{\text {st }}$ water gas shift reactor \\
\hline $\mathrm{CO}_{2}$ fraction of gas entering $2^{\text {nd }}$ water gas shift reactor \\
\hline $\mathrm{CO}_{2}$ fraction of gas exiting $2^{\text {nd }}$ water gas shift reactor \\
\hline $\mathrm{H}_{2} \mathrm{O}$ fraction of gas entering $1^{\text {st }}$ water gas shift reactor \\
\hline $\mathrm{H}_{2} \mathrm{O}$ fraction of gas entering $2^{\text {nd }}$ water gas shift reactor \\
\hline $\mathrm{H}_{2} \mathrm{O}$ fraction of gas exiting $2^{\text {nd }}$ water gas shift reactor \\
\hline
\end{tabular}

As with the primary controlled variable selection for the gasification section, the number of possible secondary controlled variable sets here is relatively small, and enumeration of all these possibilities is tractable. For this process, the optimal secondary controlled variables found are the outlet temperature of the first water gas shift reactor and the $\mathrm{CO}_{2}$ capture of the AGR. This is an intuitive set of secondary controlled variables. Most of the carbon monoxide conversion occurs within the first water gas shift reactor and a reasonable measure of that conversion is the outlet temperature as there is no associated delay with the temperature measurements as compared to the compositions. For the carbon capture, using the secondary controlled variable of $\mathrm{CO}_{2}$ capture reduces the IAE of that controlled variable loop by $60 \%$ from the case with no secondary controlled variable. 


\section{Conclusions}

A methodical, three-stage, model-based approach for primary controlled variable section has been developed. In the first stage, the focus is on a priori analysis in which the process is optimized with respect to an operational objective at the nominal operating point as well as under the identified disturbances subject to the operational and other constraints. The active constraints identified by the optimization studies are then paired with appropriate manipulated variables. A candidate set of controlled variables is then identified such that each individual candidate controlled variable is controllable in both respects, servo and regulatory. At the second stage, the Pareto-optimal controlled variable sets are selected by considering maximization of economic, i.e., self-optimizing, performance and control performance, as well as addressing issues of loop interactions and dead-time. The measures taken for self-optimizing control performance and controllability are the exact local average loss and the minimum singular value, respectively. Loop interactions are addressed by the inclusion of a constraint based upon an RGA analysis. A constraint is included to address the issue of dead-times within the loops. To solve the large-scale, constrained, multi-objective mixed integer optimization problem, a parallelized, bidirectional BB algorithm has been developed so that the optimization problem can be run in parallel on a large computer cluster to decrease the computation time. This new BB algorithm implements multiple, dynamically selected search strategies to maximize the potential gains from being run on a large computer cluster. At the last stage, a posteriori analysis is performed to ensure the economic and control performance of the controlled variable sets by using the nonlinear process model. Two possible situations are evaluated: presence or absence of optimal setpoints from a RTO.

A methodical, three-stage, model-based approach for secondary controlled variable selection has been developed. In the first stage, the focus is on a priori analysis. At this stage, an initial set of candidate secondary controlled variables and local disturbance likely to affect the process are identified. A subset selection constraint is then formulated to ensure the control performance of the resulting set of secondary controlled variables. Next, input-output models are identified for all feasible pairings, as defined by the subset selection constraint, of input to secondary controlled variable. Finally, from the identified input-output models, the IAE of the primary controlled variables for all feasible pairings of $u-y_{2}-y_{1}$ are calculated for regulatory and servo control and scaled according to the economics of the process. At the second stage, secondary controlled variable sets are selected. This is done by solving a mixed-integer constrained 
optimization problem. This optimization problem seeks to minimize economic loss associated with the control performance of the supervisory control layer while not violating controllability constraints. The objective function used in this optimization problem is the minimization of the summation of the scaled IAEs associated with a secondary controlled variable set. The IAEs calculated in the a priori analysis are used as a lookup table so as to minimize the computational expense of the optimization problem. To determine the particular pairings of the regulatory and supervisory control layers, an RGA analysis is applied. The RGA analysis, in addition to determining appropriate control pairings, is also used to apply a constraint on loop interactions associated with the regulatory and supervisory control layers. Finally, constraints are imposed to ensure the servo and regulatory control performance of the regulatory control layer. At the last stage, a posteriori analysis is performed to ensure the control performance of the controlled variable sets at off-design operations. Linearized process models are generated from the nonlinear process model at these extreme operations and the servo and regulatory control performance of the secondary controlled variables are ensured. Additional evaluations can be undertaken as needed, for example to ensure that no gain switching occurs in the regulatory control layer. From these analyses, a secondary controlled variable set that ensures good control and economic performance will be identified.

The proposed methodology for primary and secondary controlled variable selection has been applied to the large-scale, highly nonlinear AGR unit of an IGCC power plant with $\mathrm{CO}_{2}$ capture. It has been found that the proposed methodology for solving the primary controlled variable selection problem yields good results. Applying the a priori analysis to the initial set of controlled variables reduces the size of the optimization problem by over $95 \%$ and allows for the consideration of a large initial set. The search for the Pareto-optimal primary controlled variables yields 17 distinct controlled variable sets. During the a posteriori analysis, first the economic performance of the controlled variable sets is evaluated by considering a number of disturbances of various magnitudes. It is observed that during the off-design operation of the plant, some sets of controlled variables may be infeasible because the active constraints can change or there may be no manipulated variable that can control one or more controlled variables in those sets while satisfying the process constraints. While evaluating the control performance in presence and absence of an RTO, it is observed that several of the controlled variable sets lose control performance significantly at extreme operations of the process and gains of several of the identified controlled variables change direction in comparison to the nominal conditions. 
Finally two sets of optimal primary controlled variables are obtained. These sets will be used for control system design in our future work.

The proposed method for secondary controlled variable selection has been found to identify good sets that balance economic and control performance. Ten sets are desired from the set selection. Users may specify the number of desired sets. However, if too few are selected during optimization, none may be found to be feasible during the posteriori analysis. If too many solutions are desired from the optimization, the computation may become intractable. From the ten selected sets, two distinct categories are identified. In the first category, $\mathrm{CO}_{2}$ capture is controlled, via a secondary controlled variable, by manipulating the pressure of the low-pressure flash vessel. Additionally, the sets under the first category contain variables between the lean solvent flow and solvent composition within the $\mathrm{H}_{2} \mathrm{~S}$ absorber. For the controlled variables under the second category, a change in the structure of the supervisory control layer in comparison to the base case is needed. Here, the secondary controlled variables selected require the control of the $\mathrm{CO}_{2}$ capture, via a secondary controlled variable, by manipulating the semi-lean solvent flow. This particular structure provides superior control performance for $\mathrm{CO}_{2}$ capture; however, it does not improve the control performance of the lean solvent loop. Finally, theses ten controlled variable sets are examined at off-design operations to finalize the selection. Regulatory and servo control performance are investigated as well as ensuring that no gain switching occurs within the control loops. From these off-design analyses, three secondary controlled variable sets were found to be viable at all expected operational conditions of the AGR. 


\section{Suggestions for Future Work}

In this section, suggestions will be offered to help further advance the field of plantwide control.

\subsection{Primary Controlled Variable Selection}

More rigorous measures of controllability should be applied to the primary controlled variable selection procedure. The method applied in this work, minimum singular value of gain matrix, address only one issue of control performance, i.e., the worst-case control performance. Though worst-case control performance is an important consideration in the design of the control structure, it should not be the only consideration. It is suggested that for future work in this field that a partially controlled plant analysis be undertaken to determine process controllability. The benefits of the partially controlled plant analysis are that several operational conditions can be examined. In addition to the considerations of the worst-case control scenario, as currently considered, regulatory control performance can also be examined as well as the control structures sensitivities to measurement errors. These additional considerations could provide better insight into the design of the control structure rather than the examination of only the worst-case scenario.

Through the course of this work, the subset selection constraint that had been applied to primary controlled variable selection was advanced to the form that was used in the secondary controlled variable selection. It would be suggested that future work in the area of primary controlled variable selection use this new, more rigorous constraint. As the constraint used in this work for primary controlled variable selection relies primarily on process insight of the user, it is possible that valid solutions may be defined as infeasible due to lack of user insight. The subset selection constraint used here for secondary controlled variable selection can just as easily be applied to primary controlled variable selection, requiring less process insight, reducing the possibilities of eliminating possible solutions. In addition, the general form of the subset selection constraint used in secondary controlled variable selection is more flexible, allowing for many new possible applications in addition to the ones considered in this work.

\subsection{Secondary Controlled Variables}

The major assumption taken in the work on the secondary controlled variable selection procedure is that secondary controlled variables do not interact with one another. This assumption is enforced by applying tight constraints on the RGA. However, it is important to 
note that the RGA is a steady-state analysis. Therefore, even though the secondary controlled variables may not interact significantly at steady-state, transient operations may show some significant interactions. For this reason, future work in the area of secondary controlled variable selection should include the effect of loop interactions. This is expected to be computationally expensive; however, it would provide superior insights when selecting secondary controlled variables for a process.

\subsection{Control Structure Design}

The way in which the control structure design algorithm has been implemented is based upon the user defined control structures of interest to be considered. The algorithm will then find the optimal form of the desired structure. The drawback of this method is that superior control structures not examined by the user will, inevitably, be overlooked. For a more rigorous means of design of the control structure, the optimization problem must be formulated in such a way that all control structures are examined, not only those defined by the user. There are many challenges to overcome to accomplish this. The first is in the programing of an algorithm to 'understand' physically meaningful structures from those that are meaningless. As was discussed in Section 2.2.3, not all selections of elements from the Gramian interaction measures have physical meaning. For example, if we consider a diagonal pairing in the Gramian interaction matrix, that is a decentralized control structure, and add one off diagonal element, this represents the addition of a feed-forward element to the control structure, specially that the effect of one control loop is considered within another. However, if one considers the opposite case, that is we consider all elements in the Gramian interaction matrix (fully centralized control) and remove one element, the structure has no relevant physical meaning. Second, a more robust measure of controller complexity than what has been used in this work is required. The measure of controller complexity used in this work is applicable only to MPC controls. For a more rigorous control structure design procedure, a more rigorous definition of controller complexity is required. In addition, the control complexity measure used in this work does not address the complexity related to using either a LMPC or a NMPC. This is a very difficult metric to attain in particular to NMPC. Such a metric would have to be dependent upon the nonlinearity of the NMPC, the solver used to solve either the LMPC or the NMPC, etc. Furthermore, determining rather a LMPC or a NMPC would be used for a particular controlled/manipulated variable set would add a new dimension to the control structure design problem, possibly increasing the 
solution time considerably. Finally, the formulated optimization problem must be solved. In this work, mixed integer optimization problem have been solved using a branch and bound algorithm; however, it is not guaranteed that a formulated optimization problem which addresses the above raised issues can be solved via this method.

The control structure design methodology should also address issues related to process economics. The methodology currently as currently applied considers the control performance of all controlled variables to be of equal importance and the relative gains of all the input variables to be of the same magnitude. This is similar to the analysis that is attained by applying an RGA analysis. However, from an economic point of view, all controlled variables are not of equal importance, indeed, nor are the manipulated variables. A more rigorous methodology should be devised which addresses the relative importance, from an economic perspective, of the primary controlled and manipulated variables.

In relation to the issue of MPC tuning, a nonlinear approach should be undertaken. In this work, the economics of the process is assumed to be a linear function of the controlled variables. This is not the case for real world processes. For the tuning of the MPC, this nonlinearity should be considered. In the case presented in this work, $\Theta_{i}$ within Equation 52 will be a nonlinear function of the state of the process rather than a static, linear relation.

Finally, a major issue related to the field of plantwide control, not yet investigated in any work known to the author, is related to the issue of sequential vs. simultaneous control design. To illustrate the point, consider the work present in this paper. A rigorous, methodological approach to the development of a control structure for the AGR unit and gasification section of an IGCC power plant has been presented. However, many other units exist within in the IGCC power plant. Obviously, the ideal solution would be to simultaneously design the entire control structure for the IGCC power plant; however, given the size of the process, the number of candidate controlled variables, manipulated variables, measured variables, secondary controlled variables, etc., makes such an undertaking impractical, if not impossible. Therefore, a systematic and rigorous means of designing such a control structure must be addressed. This method would have to rely on an application of both a sequential and simultaneous design methodology to be applied to real world, large scale processes. All works that have been found within the open literature, including this work, focus entirely on the simultaneous design method. However, as a sequential method must be developed to allow the plantwide control methodology to become applicable to large scale systems. The sequential method must address how subsets of an entire 
process will be defined, how the controls between the processes will be defined, and how the boundaries of the subset processes will be reconciled. In addition, the integration of the sequential and simultaneous needs to be developed. It may be found that after the control structure is designed simultaneously for each of the subsystems that the integrated system does not perform satisfactorily. Methods need to be investigated to address these future concerns for plantwide control design. 


\section{References}

Akaike, H. A new look at the statistical model identification. IEEE Trans. Auto. Control 1974, 19, 716723

Alonso, A.A. and Ydstie, B.E., "Stabilization of distributed systems using irreversible thermodynamics," Automatic, 37, 2001, pp. 1739-1755

Aspen Plus Dynamics. AspenTech. < http://www.aspentech.com/products/aspen-dynamics.aspx>

Araujo, Antonio C. B., Eduardo S. Hori, and Sigurd Skogestad. "Application of Plantwide Control to the HDA Process. II - Regulatory Control." Ind. Eng. Chem. Res., 2007: 5159-5174.

Araujo, Antonio C.B., Marius Govastsmark, and Sigurd Skogestad. "Application of plantwide control to the HDA process. I - steady-state optimization and self-optimizing control." Control Engineering Practice, 2007: 1222-1237.

Bhattacharyya, D., R. Turton, and S. Zitney. "Steady-state simulation and optimization of an integrated gasification combined cycle (IGCC) power plant with CO2 Capture." Ind. Eng. Chem. Res., 2011: 1674-1690.

Birk, W., and A. Medvedev. "A note on Gramian-based interaction measures." Proceedings of European Control Conference. Cambridge, UK, 2003.

Bristol, E.H. "On a new measure of interaction for multivariable process control." IEEE Trans. Automatic Control, 1966: 133-134.

Bucklin, R.W., and R.L. Schendel. "Comparison of Fluor Solvent and Selexol Processes." Energy Progress, 1984: 137-142.

Cao, Yi, and Vinay Kariwala. "Bidirectional branch and bound for controlled variable selection Part I. Principles and minimum singular value criterion." Computers and Chemical Engineering, 2008: 2306-2319.

Chen, X.-W. "An improved branch and bound algorithm for feature selection." Pattern Recognition Letters, 2003: 1925-1933.

Conley, A., and M.E. Salgado. "Gramian based interation measure." Proceedings of the 39th IEEE Conference on Decision and Control. Sydney, Australia, 2000. 5020-5022.

Crainic, Teodor Gabriel, Bertrand Le Cun, and Catherine Roucairol. "Parallel Branch-and-Bound Algorithms." In Parallel Combinatorial Optimization, by El-Ghazali Talbi, 1-28. John Wiley and Sons, Inc., 2006.

DOE/NETL. "Cost and performance baseline for fossil energy power plants, Volume 1: Bituminous coal and natural gas to electricity." Morgantown, WV, 2007.

Foss, A.S. "Critigue of chemical process control theory." AIChE Journal, 1973: 209-214.

Halvarsson, Bjorn. Interaction Analysis in Multivariable Control Systems Applications to Bioreactors for Nitrogen Removal. Uppsala: Uppsala University, 2010.

Halvorsen, I.J, S. Skogestad, J. Morud, and V. Alstad. "Optimal selection of control variables." Ind. Eng. Chem. Res., 2003: 3273-3284.

Havre, K., and S. Skogestad. "Selection of variables for regulatory control using pole vectors." Proceedings of the IFAC Symposium. Corgu, Greec, 1998. 614-619.

Jones, D., D. Bhattacharyya, R. Turton, and S.E. Zitney. "Rigorous Kinetic Modeling and Optimization Study of a Modified Claus Unit for an Integrated Gasification Combined Cycle (IGCC) Power Plant with CO2 Capture." Ind. Eng. Chem. Res., 2012: 2362-2375.

Jones, D., Bhattacharyya, D., Turton, R., and Zitney, S. E. "Optimal Selection of Primary Controlled Variables for an Acid Gas Removal Unit as part of an IGCC Plant with CO2 Capture" Proceedings of American Controls Conference, 2013

Kariwala, V., and Y. Cao. "Local self-optimizing control with average loss minimization." Ind. Eng. Chem. Res., 2008: 1150-1158.

Kariwala, Vinay, and Yi Cao. "Bidirectional Branch and Bound for Controlled Variable Selection Part III: Local Average Loss Minimization." IEEE Transactions on Industrial Informatics, 2010: 1-2. 
Kariwala, Vinay, and Yi Cao. "Bidirectional branch and bound for controlled variable selection. Part II: Exact local method for self-optimizing control." Computers and Chemical Engineering, 2009: 1402-1412.

Kariwala, Vinay, and Yi Cao. "Branch and bound method for multiobjective pairing selection." Automatica, 2010: 932-936.

Karmarkar, N. "A New Polynomial-Time Algorithm for Linear Programming.” Combinatorica 4 (4), 1984, 373-395

Kinnaert, M. "Interaction measures and pairing of controlled and manipulated variables for multiple-input and multiple-output systems: A survey." Journal A, 1995: 15-23.

Konda, N. V. S. N. Murthy, Rangaiah, G.P., and Krishnaswamy, P. R., "Plant-wide control of industrial process: An integrated framework of simulation and heuristics" in Ind. Eng. Chem. Res., 2005, pp. 8300-8313

Kreindler, E., and P.E. Sarachik. "On the concepts of controllability and observability of linear systems." IEEE Transactions on Automatic Control, 1964: 129-136.

Kothare, M.V., Shinnar, R., Rinard, I., and Morari, M., "On Defining the Partial Control Problem: Concepts and Examples," AIChE Journal, 46, 12, 2000, pp. 2456-2474

Larsson, T., M.S. Govatsmark, S. Skogestad, and C.C. Yu. "Control Structure Selection for Reactor, Separator, and Recycle Processes." Ind. Eng. Chem. Res, 2003: 1225-1234.

Luyben, W., Tyreus, B., and Luyben, M., Plantwide Process Control. New York, USA: McGraw-Hill, 1998

Maarleveld, A., and J.E. Rijnsdrop. "Constraint control of distillation columns." Automatica, 1970: 51-58.

Morari, M., G. Stephanopoulos, and Y. Arkun. "Studies in the synthesis of control structures for chemcial processes. Part I. Formulation of the problem. Process decomposition and the classification of the control task. Analysis of the optimizing control structures." AIChE Journal, 1980: 220-232.

Narendra, P., and K. Fukunaga. "A branch and bound algorithm for feature subset selection." IEEE Transactions on Computers, 1977: 917-922.

Nett, C.N. "A quantitative approach to the selection and partitioning of measurements and manipulations for the control of complex systems." Caltech Control Workshop. Pasadena, 1989.

Panahi, M., and S. Skogestad. "Economically efficient operation of CO2 capturing process part I: Selfoptimizing procedure for selecting the best controlled variables." Chemical Engineering and Processing, 2011: 247-253.

Salgado, M.E., and A. Conley. "MIMO interaction measure and controller structure selection." Int. J. Control, 2004: 367-383.

Samuelsson, Par, Bjorn Halvarsson, and Bengt Carlsson. "Interaction Analysis and Control Structure Selection in a Wastewater Treatment Plant Model." IEEE Transactions on Control Systems Technology, 2005: 955-965.

Shinnar, R., "Chemical Reactor Modeling for purposes of controller design," Chem. Eng. Com., 9, 73, 1981

Skogestad, S. "Plantwide Control: The search for the self-optimizing control structure." Journal of Process Control, 2000: 487-507.

Skogestad, S., and I. Postlethwaite. Multivariable Feedback Control. Chichester, UK: John Wiley \& Sons, 1996.

Skogestad, Sigurd. "Control structure design for complete chemical plants." Computers and Chemcial Engineering (Sigurd Skogestad, 'Control structure design for complete chemical plants' Computers and Chemcial Engineering 28 (2004) 219-234), 2004: 219-234.

Wittenmark, B., and M.E. Salgado. "Hankel-norm based interation measure for input-output parings." Proceedings of the 2002 IFAC World Congress. Barcelona, Spain, 2002.

Yelchuru, R. and Skogestad, S., "Convex formulations for optimal selection of controlled variables and measurements using Mixed Integer Quadratic Programming" in Journal of Process Control, 2012, pp. 995-1007 


\section{Appendix A: Parallelized Bi-Directional Branch and Bound Algorithm}

A branch and bound algorithm has been selected for solving the control variable selection problem. The branch and bound approach has an important advantage over genetic algorithms, which can also be used for solving this problem. The advantage is that the solution attained by branch and bound is guaranteed to be the globally optimal solution. There exist branch and bound algorithms within the open literature which can be used for solving this problem (Cao \& Kariwala, 2008, 2009, and 2010) and they have been investigated. However, the branch and bound algorithm developed by Cao and Kariwala (Cao \& Kariwala, 2008) has two limitations. Their algorithm is not capable of constraint handling nor can it be parallelized. Due to these limitations, a new branch and bound algorithm has been developed, which closely follows the work of Cao and Kariwala. The developed algorithm is capable of constraint handling and has been parallelized on a cluster of processors. This algorithm is being coded within MATLAB ${ }^{\circledR}$. Additionally, efforts have been made in the development of the code so as not to lose generality, ensuring the code can be used to solve a variety of mixed-integer nonlinear problems.

The first to propose the use of branch and bound methods for a subset selection problem was Narendra and Fukunaga (Narendra \& Fukunaga, 1977). Branch and bound is used for subset selection problems because, of all the currently available methods, only branch and bound and brute force can guarantee a globally optimal solution (Chen, 2003). Branch and bound, however, is significantly computationally less expensive than an exhaustive, brute force search method where all possible solutions are evaluated. This is not only an efficiency issue, but also a problem of practicality. For the subset selection problem being investigated for this work, the problem is that of choosing 6 from 320, $\left(\begin{array}{c}320 \\ 6\end{array}\right)$, which gives over 1.4 trillion possibilities. An exhaustive search for the globally optimal solutions using brute force enumeration would take approximately 450 years, assuming the evaluation of each solution took $0.01 \mathrm{~s}$. It should be noted that $0.01 \mathrm{~s}$ is the approximate time it takes $\mathrm{MATLAB}^{\circledR}$ to calculate the two objective functions for a $6 \times 6$ matrix. The problem, of course, will take significantly longer as the objective function evaluation becomes more computationally expensive.

Figure A.1 shows the basic concept of branch and bound. Figure A.1 shows a theoretical

solution tree for a $\left(\begin{array}{l}6 \\ 2\end{array}\right)$ problem and will be used as an example of the branch and bound algorithm that has been developed. In this example, $J$ is the objective function which is to be 
maximized. The large, original problem is called the root, located at level 0 . This problem is divided into two smaller problems. These two smaller problems, shown at level 1, span the space of their parent node and have no intersection. Since the two branches span the parent and do not intersect one another, it is said that this branching strategy is complete and without redundancies. These two smaller problems can likewise be split into four smaller problems, at level 2. Like with the transition from level 0 to level 1 , the transition from level 1 to level 2 is both complete and without redundancies. The particular branching method shown in Figure A.1 is called 'bidirectional' because one branch has been expanded or branched up, i.e., an element has been added to the set, and another has been shrunk or branched down, i.e., an element has been removed from consideration. Note that the element to the far right in level 2 is a so called 'terminal branch' because it contains only 1 subset and thus cannot be further branched, i.e., it is a solution. This terminal branch is evaluated using the selection criteria and is used as a bound. Since the selection criterion is to be maximized, this bound is the best available lower limit of the objective function, otherwise known as the incumbent, which exists for the globally optimal solution. This means that the globally optimal solution will be either greater than or equal to this bound, but it cannot be less. Now, all existing nodes are evaluated using the selection criterion. Since the selection criterion is monotonic, any node whose selection criterion is lower than that of the current bound should be 'pruned' so that none of its subsets need be considered further. Note that there exist two such selection criteria for the evaluation of a non-terminal node. These correspond to an upward evaluation of the criterion, i.e., the criterion is evaluated with relation to the elements added to that branch, and a downward evaluation of the criterion, i.e., the criterion is evaluated with relation to all the elements that may be added to the subsets of that node. If either of the evaluations is less than the currently available bound, that branch cannot contain the globally optimal solution and should be pruned. For simplicity, only the smaller of the two are shown in Figure A.1. In the example illustrated in Figure A.1, the branch to the far left can be pruned because its criterion evaluation is lower than the bound, therefore the solution cannot be contained within that branch. This procedure continues until no branches exist that need be evaluated. 


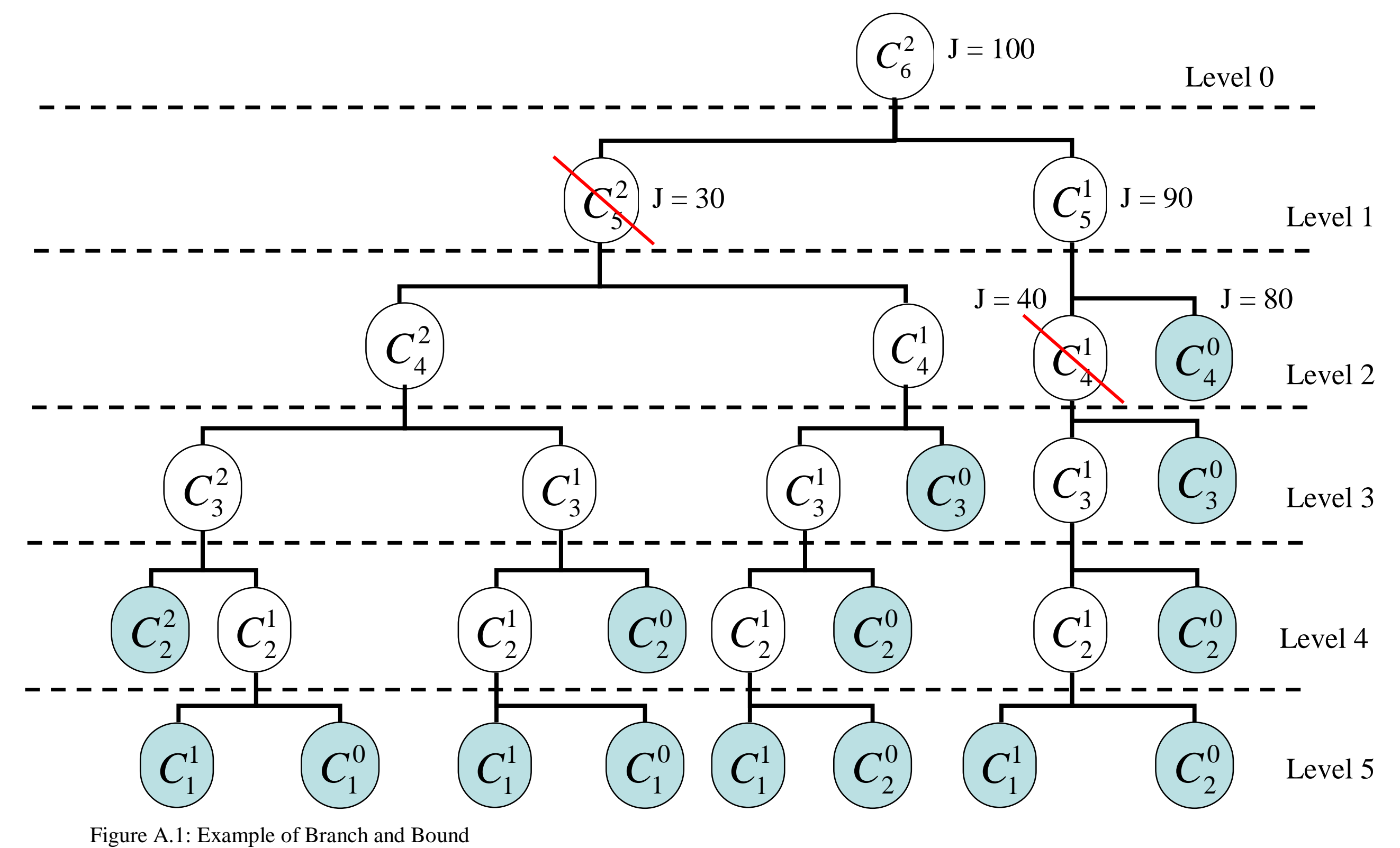

Figure A.1: Example of Branch and Bound 
The above is a simple representation of a branch and bound algorithm. Several issues still remain and need to be addressed. For example, the branching strategy and search strategy that are used in the algorithm need to be determined. The branching strategy is concerned with which variable will be added to a node in one branch and subsequently removed from the other node. To illustrate the importance of the branching strategy, it has been found that a good branching strategy can reduce computation time by as much as $45 \%$ as compared to a random branching strategy. Specific branching strategies exist for particular objective functions, however, no generalized branching strategy could be found within the open literature. Since it is desired that this code remain generic and applicable for any objective function, a generalized branching strategy has been developed. To define the strategy, the objective function(s) are evaluated for each individual element and their values are stored. The strategy is then to select elements which have the smallest objective function value and branch the node using that element. For nodes that do not contain a particular element, that element's value is set to infinity. The logic behind this strategy is to attempt to get the optimal solutions into the smallest node; namely, the node that has had an element added.

The next issue to be addressed is the search strategy. Choosing which node to branch is not obvious; however, three prominent strategies exist: breadth first, depth first, and best first. Each has advantages and disadvantages and which strategy is chosen depends largely on the particular problem. The breadth first strategy uses the largest existing node to branch. The depth first strategy evaluates the smallest existing node first. The best first strategy evaluates the node that has the best current objective function value. The advantage with a breadth first strategy is that it is less likely that time will be spent evaluating nodes that do not contain the global optimum; however, at the same time, its disadvantage is that less effort will be spent on nodes that may contain the globally optimal solution. A depth first search is advantageous as it will deeply search in a node. If that node contains the globally optimal solution, the best possible bound information is found and pruning of the remaining nodes will be accelerated. Additionally, the depth first search is the least memory intensive of all the strategies as fewer nodes exist at any one time. However, the disadvantage with a depth first approach is that if the node selected does not contain the global optimal, the bound information found will be poor and pruning will be slow resulting in more enumerations. Finally, the best first strategy is advantageous as it will 
quickly find good bounds that will allow for quick pruning. Additionally, large nodes with poor objective function values will not be branched and may be pruned as better bounds become available, thus reducing enumeration. However, this is a very memory intensive strategy. As the "best" current nodes will likely consist of the largest currently available nodes and those are the nodes that will be branched. When branched, the node's objective function will be worse, and thus another node may be better and will, therefore, be branched. This can create a large number of nodes that must be tracked. Another disadvantage is that, in general, the evaluation of the bounding function for a large node is more computationally expensive than the evaluation of a smaller node.

The branch and bound algorithm has been developed to be a multi-objective optimizer. The code will find the Pareto front of any two arbitrary objective functions. This is done following the work of Kariwala and Cao, 2010. To accomplish this, only the pruning criteria need be changed. From the example shown in Figure A.1, where only one objective is considered, pruning occurs if either Equation A.1 or A.2 is true. For finding the Pareto front, pruning occurs if the condition shown in Equation A.3 is not satisfied. Figure A.2 shows a graphical representation of this pruning criterion. From Figure A.2, consider that points A, B, C, and D constitute the current Pareto front that has been found. Now, a new terminal node is evaluated, shown as point E, this point "dominates", i.e., it is superior with respect to both objectives, points B, C, and D. Therefore, points B, C, and D are no longer members of the Pareto front and are discarded. Now, a non-terminal node is evaluated and has objective function values representing point $F$. As point $\mathrm{A}$ dominates point $\mathrm{F}$, the node represented by point $\mathrm{F}$ is pruned. For completeness, consider another non-terminal node with objective function values representing point $G$, this point is not dominated by any existing member of the Pareto front and is therefore not pruned. In short, any nodes with objective function values that place it within the area of the dashed lines are pruned.

$$
\begin{aligned}
& J\left(X_{S}\right) \geq B, \quad \forall X_{n} \subseteq X_{S} \\
& J\left(X_{S}\right) \geq B, \quad \forall X_{n} \supseteq X_{S} \\
& \exists s, t \in N_{q}: J_{S}\left(P_{n}^{i}\right)<J_{s}\left(P_{n}^{j}\right), \quad J_{t}\left(P_{n}^{i}\right)>J_{t}\left(P_{n}^{j}\right)
\end{aligned}
$$




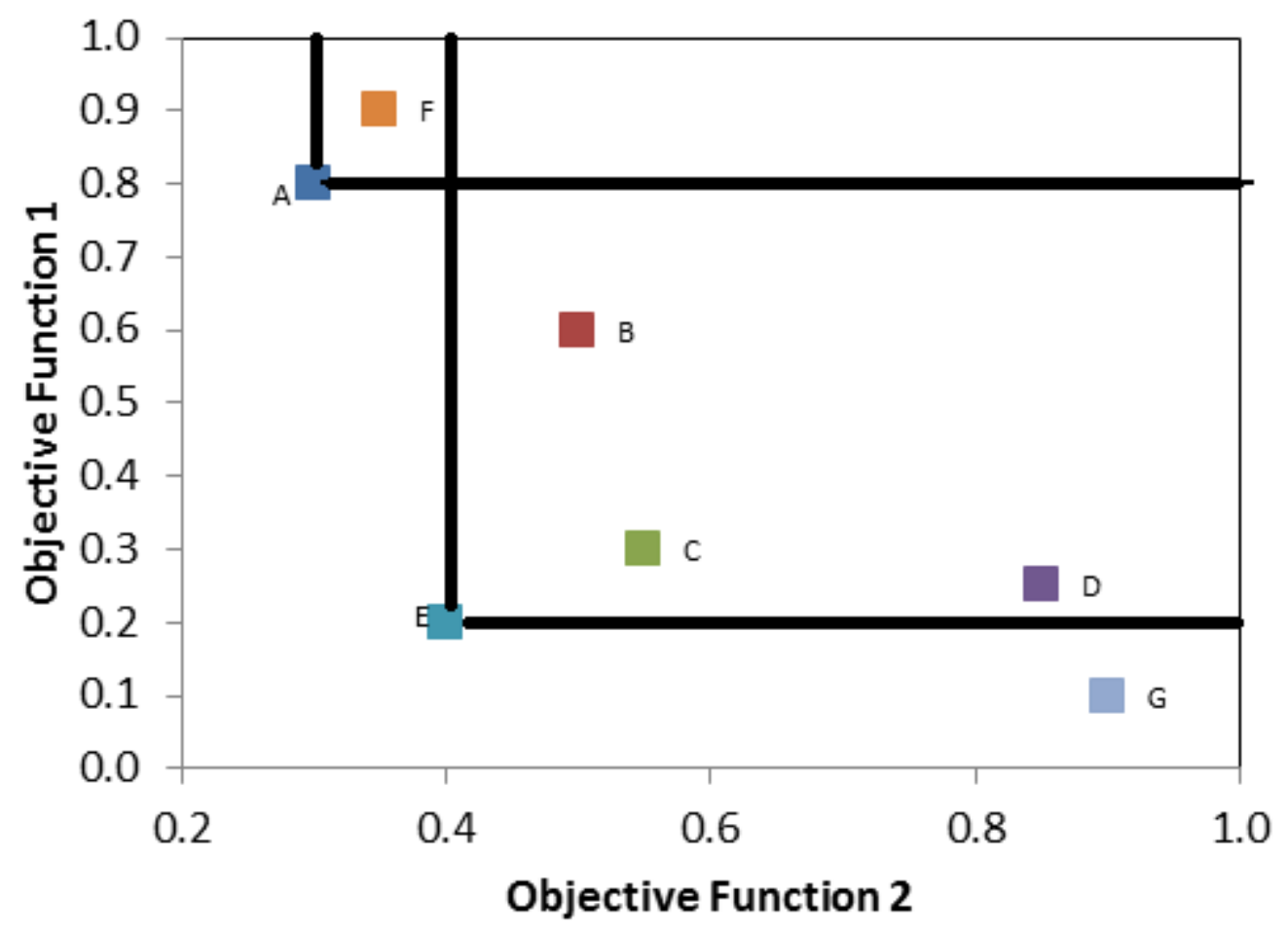

Figure A.2: Example of Pruning Rule for finding the Pareto Front

Due to the nature of the above formulation, (Cao \& Kariwala, 2008) a 'fast pruning' algorithm cannot be used. Because of this, computation time increases. To address this issue, the above algorithm has been made capable of running in parallel on a cluster of machines. Figure A.3 shows the method that has been used to parallelize the problem. As has been mentioned previously, the branching strategy that is used results in two complete, non-redundant nodes. Because of this, the two nodes generated from the original parent may be considered as two independent problems. Therefore, every node may be considered as a sub-problem that is stored at the 'head node' and distributed to 'workers' to be solved in parallel. When a worker has solved the problem or a termination criterion is met, the worker sends the results back to the head node where the results from all the workers are gathered, processed, and the global bound information is updated. Any nodes that were not evaluated by the worker are stored with all remaining nodes at the head-node. The head-node then redistributes nodes to the workers to be solved. This process continues until all nodes have been evaluated or pruned. It is important to note that although all workers may refer to the global bound information, they may not update the global bound information. To address this issue, each worker has its own local bounding 
information that, at the start of the process, is the same as the global bound information. If local bounding information is available to a worker and it is superior to the global bounding information, the worker will use its own local bound information over the global.

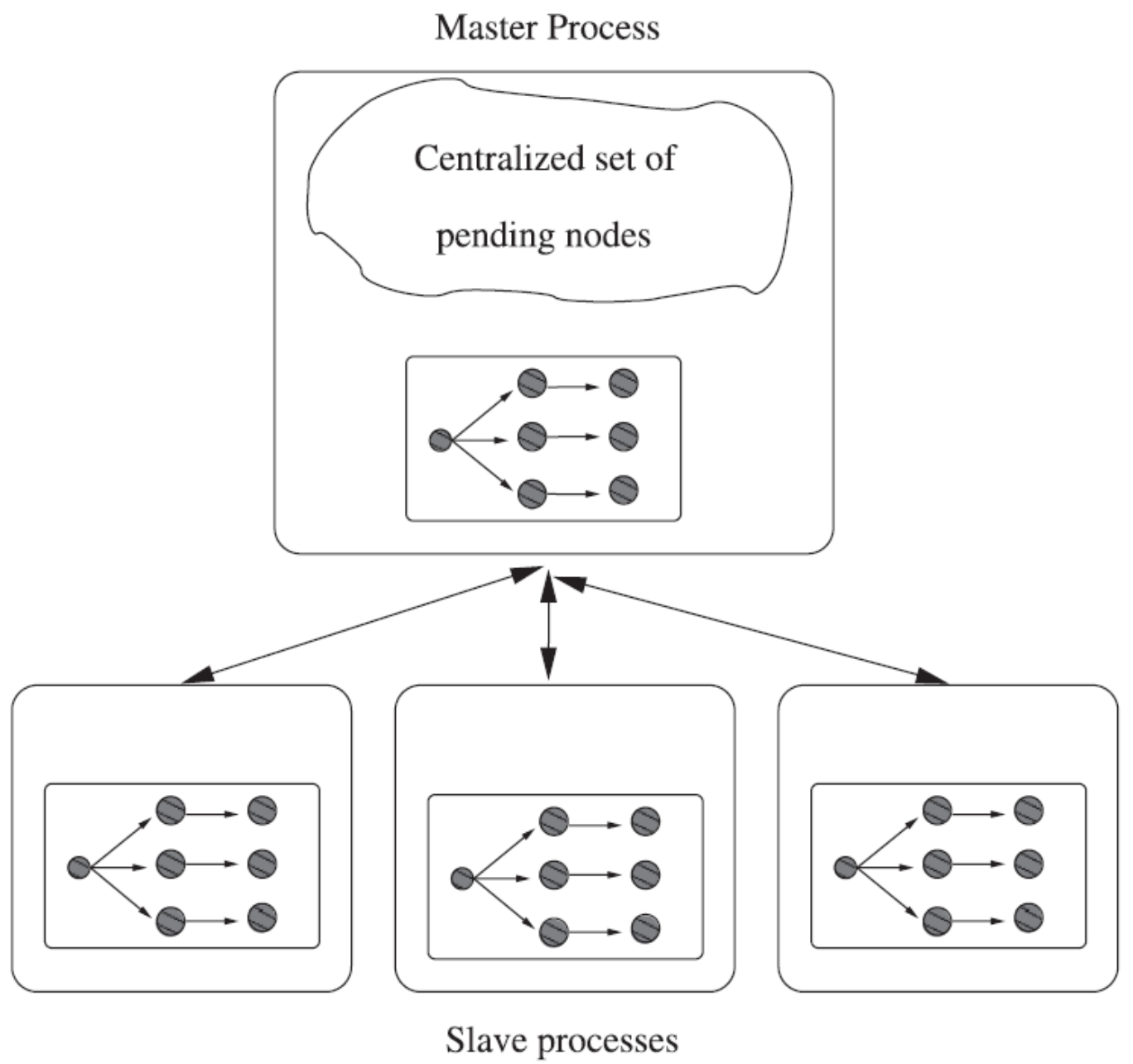

Figure A.3: Parallelizing Method for Branch and Bound Algorithm (Crainic, Le Cun and Roucairol 2006)

Inefficiency arises from this approach. The inability to share bound information between workers results in wasted computational effort evaluating branches that could have been pruned had the worker had access to the better bound information. Another concern is keeping workers active as often as possible. By the nature of the parallelizing strategy that has been chosen, when a worker completes its task, it will wait for an additional task. However, no new tasks will be made available until all workers have finished their tasks. Keeping all the workers active is, 
obviously, important for maximizing the gains from the cluster. Essentially, the first concern is maximizing useful work and the second is maximizing total work.

It should be noted that there exist the so called 'anomalies of parallelized branch and bound' (Crainic, Le Cun, \& Roucairol, 2006). There are two such anomalies. The first is a favorable speed-up anomaly and the second is an unfavored slow-down anomaly. As an example, if we consider a problem that would take 100 mins to solve in series on one processor, adding a second processor would, intuitively, result in a computation time of 50 mins if the parallelization where $100 \%$ efficient. However, with branch and bound, it is possible to have an improvement that is greater than this, the so-called "speed-up" anomaly, and it is possible that the computation time may increase, the slow-down anomaly. These anomalies are present in branch and bound due to the search path that is taken by the algorithm. The efficiency of branch and bound is based largely on the quality of the available bound. However, the bound that is available at any point in time is dependent upon the search path that has been taken. With a parallel branch and bound algorithm, the search path taken will differ from the path that was taken when run in series with only one machine and, more generally, it will differ based upon the number of workers available. Therefore, the bound information found may be better or worse than the bound information that would have been found had the problem been solved in series, thus resulting in these anomalies. Therefore, any parallelized branch and bound algorithm must minimize the risk of the slowdown anomaly and, if possible, increase the chance of the speed-up anomaly.

The basic method used to parallelize the branch and bound algorithm is shown in Figure A.3, however, there are several important issues to be resolved with this approach. The first is to determine which node(s) should be sent to the workers. Second, is to determine what search strategy the workers should employ. Third, how long should each worker be allowed to solve its problem should be determined? The first issue relates to which nodes should be sent to the workers. It has been decided that the head node will send all existing nodes to the workers. The second issue relates to the search strategy that the workers will employ. This is decided by the head-node. If the head-node has more than 30,000 nodes to store, workers will engage in a depth-first search. Otherwise, the workers will employ both a best-first and depth-first search. The worker is allowed so many iterations using a best-first search and after that number of iterations, it will switch to a depth-first search. Finally, how long each worker is allowed to work on a problem needs to be determined. It has been decided that each worker will be allowed 
to work on their problem for 2 mins before being terminated so that the problem can be redistributed and global bound information can be updated.

Finally, as the branch and bound algorithm is searching for the Pareto front, there is not a single objective it is trying to achieve. For the controlled variable selection problem, there are two objectives. This introduces several challenges. First, how many solutions that exist are unknown. Second, as there are two objectives that are to be optimized, defining what node is 'best' is not obvious. Additionally, multiple branching strategies may be needed to have acceptable performance. To address these issues, the Pareto front has been considered as two separate regions to be explored. A generic Pareto front and the two regions considered are shown in Figure A.4. Any nodes that fall within Region 1 are considered to be nodes which are 'best' in terms of Objective 1. Because of this, they are branched in terms of Objective 1. Likewise, nodes which fall within Region 2 are considered to be nodes which are 'best' in terms of Objective 2 and are branched accordingly.

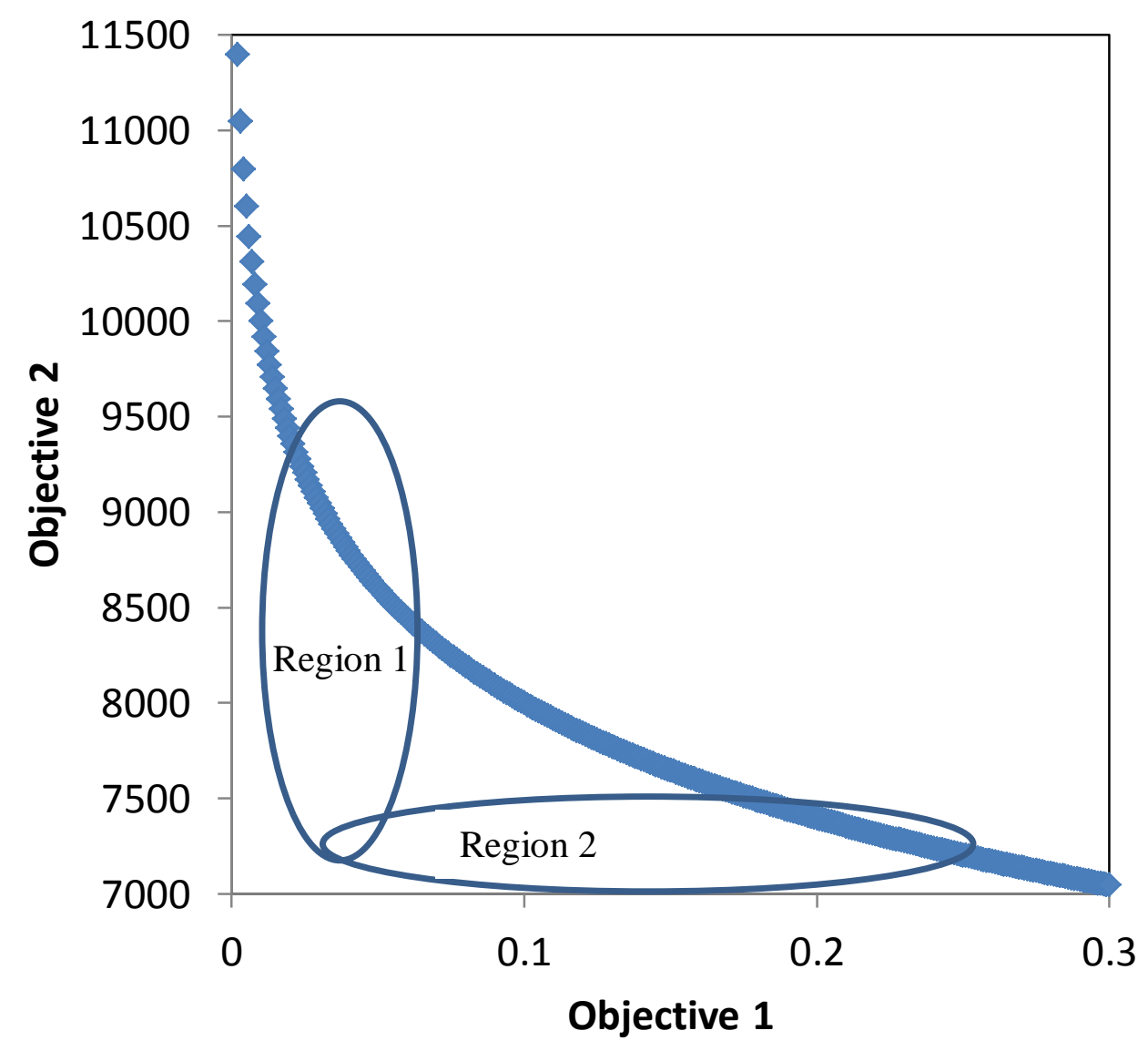

Figure A.4: Region approach used for Pareto front Calculation 